\title{
THE SPIN STRUCTURE OF THE PROTON IN THE RESONANCE REGION
}

\author{
Renee H. Fatemi \\ Sterling, VA
}

B.S., University of Virginia, 1995

A Dissertation Presented to the Graduate

Faculty of the University of Virginia

in Candidacy for the Degree of Doctor of Philosophy

Department of Physics

University of Virginia

January, 2002 


\section{Abstract}

Inclusive double spin asymmetries have been measured from $\vec{p}\left(\vec{e}, e^{\prime}\right)$ using the CLAS detector and a polarized ${ }^{15} \mathrm{NH}_{3}$ target at Jefferson lab in 1998. The virtual photon asymmetry $\mathrm{A}_{1}$, the longitudinal spin structure function, $g_{1}\left(x, Q^{2}\right)$, and the first moment, $\Gamma_{1}^{p}$, have been extracted for a $Q^{2}$ range of $0.15-2.0 \mathrm{GeV}^{2}$. These results provide insight into the low $Q^{2}$ evolution of spin dependent asymmetries and structure functions as well as the transition of $\Gamma_{1}^{p}$ from the photon point, where the Gerasimov, Drell and Hearn Sum Rule is expected to be satisfied, to the deep inelastic region. 


\section{Acknowledgements}

There are places I remember, all my life... though some have changed. Some forever not for better

some have gone and some remain

All these places have their moments, with lovers and friends... I still can recall, some are dead and some are living...

In my life, I've loved them all

- Excerpt from In My Life by the Beatles

I thank my advisors Don Crabb and Ralph Minehart for their patience, guidance and willingness to pass on their expertise. I believe it is a rare and fortunate opportunity to work with two advisors whom I respect on both a personal and professional level. I also thank Cole Smith for freely giving his time to help me with computer problems, programming and analysis projects. I thank all the past and present members of the Polarized Target Group here at UVa. I have found what I hope to be lifelong friendships while also learning from this group of intelligent and motivated people. In particular I thank Todd Averett, Stephen Bueltmann, Paul McKee, Dustin McNulty, Al Tobias and Dave Zimmermann. I thank Yelena Prok for lively discus- 
sions on physics, God, politics and good food. And of course I owe much gratitude to Chris Harris, my target companion, for many years of laughter, good advice, honesty and twizzlers.

There are people who are not affiliated with the University of Virginia but have proven to be an invaluable resource to me during my time as a graduate student. I thank the group from Genova, in particular Raffaella DeVita, for their wonderful companionship as well as dedication to the Polarized Target and EG1 analysis. I thank Sebastian Kuhn and Tony Forest for their quick and thorough responses to all my questions. Volker Burkert, Gail Dodge, Keith Griffioen and Peter Bosted also provided insight and assistance for what seemed to be endless analysis tasks. I would also like to thank Kim Haddock for the many nights she spent in the Hall-B counting house working on the target software with me.

There are always people, though not directly involved in research, that provide assistance and necessary support. The short list consists of Teresa Marshall, Tammy Shifflet, Faye Safely, Bobby Floyd, Gwen Fantino, Jim Shea, Suzie Garret, Pam Joseph, Brenda White, Brian Wright and Rodger Ashley. Over the years they have insured that parts are ordered, paychecks are deposited and books secured as well as providing friendship and good spirit. Then of course I thank Amber Mierisch. Although we didn't follow the same path in physics we took classes, ate meals out of the vending machines, did homework and studied for the qualifier together. She is a wonderful friend who taught me $\mathrm{E} \& \mathrm{M}$ and for that I am always grateful. In the interest of brevity there are many people who will go unmentioned here ... friends and former teachers who have fostered a community which enabled me to pursue my interests and given me much joy in the process. Please know that although your 
names are not in print here, you and your families are deeply appreciated.

Finally I thank my parents Beverly and Howard Miller for what seems to be boundless enthusiasm and support. My mother, by example, has given me the gift of a strong work ethic and the belief that any problem can be solved if I think about it long enough. I thank my father, William Hutchins, for showing me that I must live in the moment and for imparting the wisdom that at times risks are necessary in order to gain happiness. As for my husband Darius, it is hard to express in words all I owe to you. You have been on this journey with me from the start and have always understood the independence and freedom I needed to follow my research while remaining completely emotionally supportive. Thank you for these gifts and your wonderful, ironic sense of humor, without which I would have never been able to enjoy this ride. 


\section{Contents}

1 The Quark Review 1

2 Formalism $\quad 7$

2.1 Electron-Proton Scattering Kinematics . . . . . . . . . . . 7

2.2 Electron-Nucleon Cross-Sections . . . . . . . . . . . . . . . 10

2.3 Virtual Photon Scattering . . . . . . . . . . . . 14

2.4 Quark Distributions. . . . . . . . . . . . 17

2.5 Sum Rules . . . . . . . . . . . . . . . . . . 20

2.6 Gerasimov-Drell-Hearn Sum Rule . . . . . . . . . . . . . 23

2.7 Low $Q^{2}$ Models for $g_{1}^{P}$ and The First Moment . . . . . . . . . . 24

2.7.1 The $Q^{2}$ Evolution of $g_{1}^{P} \ldots \ldots \ldots \ldots$

2.7.2 The $Q^{2}$ Evolution of $\Gamma_{1}^{P} \ldots \ldots \ldots 28$

3 Experimental Setup $\quad 34$

3.1 The Accelerator and Polarized Electron Beam . . . . . . . . . . 36

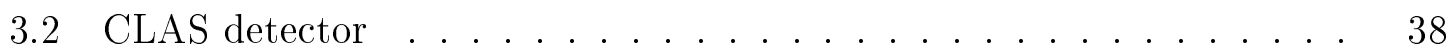

3.2.1 Torus Magnet . . . . . . . . . . . . . . 40

3.2.2 Drift Chambers ................... 41 
3.2.3 Time of Flight Detector . . . . . . . . . . . . . . 42

3.2.4 C̆erenkov Detector ................. 44

3.2.5 Electromagnetic Calorimeter ............. 45

3.2.6 Data Acquisition and Trigger System . . . . . . . . . . . 49

3.3 Polarized Target . . . . . . . . . . . . . . 52

3.3.1 Theory of Solid Polarized Targets . . . . . . . . . . . . 54

3.3.2 Dynamic Nuclear Polarization . . . . . . . . . . 55

3.3.3 The Equal Spin Temperature Theory . . . . . . . . . . 57

3.3.4 The Target Material ................ 58

3.3.5 The Target Magnet and Cryostat .............. 60

3.3.6 The Helium-4 Evaporation Refrigerator . . . . . . . . . . 65

3.3.7 The Target Insert . . . . . . . . . . . . . . 68

3.3.8 The Microwave System . . . . . . . . . . . . . 71

3.3.9 The Principles Behind Nuclear Magnetic Resonance . . . . . . 72

3.3.10 Experimental Setup of the NMR System . . . . . . . . . . 74

3.3.11 NMR signal Analysis . . . . . . . . . . . . . . 77

3.3.12 Polarized Target Software . . . . . . . . . . . . 80

4 Data Analysis $\quad 81$

4.1 Data Acquisition and Storage . . . . . . . . . . . . 81

4.2 Detector Calibration . . . . . . . . . . . . . . 82

4.3 Track Reconstruction . . . . . . . . . . . . . . 82

4.3.1 Hit Based Tracking . . . . . . . . . . . . . 83

4.3.2 Start Time Determination . . . . . . . . . . . 83

4.3.3 Time Based Tracking . . . . . . . . . . . . 84 
4.4 Helicity Information and HelP Tables . . . . . . . . . . . 86

4.5 DST Production . . . . . . . . . . . . . . . 88

4.6 Momentum Corrections . . . . . . . . . . . . . . . 89

4.7 Electron Identification . . . . . . . . . . . . . . . . 91

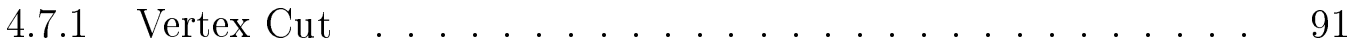

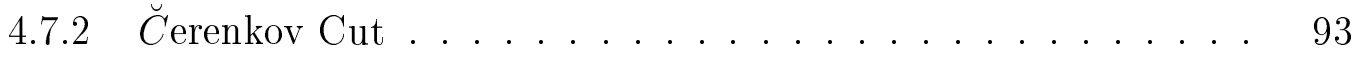

4.7.3 Electromagnetic Calorimeter Cuts . . . . . . . . . . 94

4.8 Fiducial Cuts ......................... 96

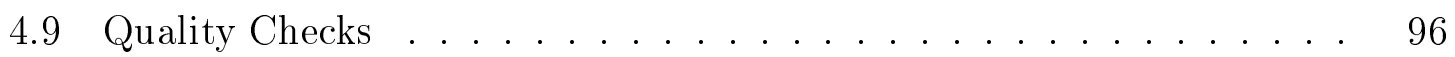

4.9.1 ${ }^{15} \mathrm{NH}_{3}$ Elastic Asymmetry . . . . . . . . . . . . . . 97

4.9.2 Beam Charge Asymmetry _. . . . . . . . . . . . 97

4.9 .3 Detector Stability . . . . . . . . . . . . . 97

4.9.4 $\mathrm{C}_{12}$ and $\mathrm{NH}_{3}$ asymmetry below elastic peak . . . . . . 101

4.9.5 $\mathrm{C}_{12}$ and $\mathrm{NH}_{3}$ Fiducial Regions . . . . . . . . . . 101

4.10 Models . . . . . . . . . . . . . . . . . 101

4.11 Background subtraction . . . . . . . . . . . . 102

4.12 Beam and Target Polarization . . . . . . . . . . . . . 109

4.13 Pair Symmetric Correction . . . . . . . . . . . . . 115

4.14 Radiative Corrections . . . . . . . . . . . . . 118

4.15 Live-time Correction . . . . . . . . . . . . . . . 121

4.16 Polarized ${ }^{15} N$ Correction . . . . . . . . . . . . . . . 122

4.17 Systematic Errors . . . . . . . . . . . . . . . 124

4.17.1 Background Subtraction . . . . . . . . . . . 125

4.17.2 Beam $\times$ Target Polarization . . . . . . . . . . 126 
4.17.3 Pair Symmetric, Live-time and ${ }^{15} N$ Correction . . . . . . . . 126

4.17.4 Models ...................... 126

4.17.5 Radiative Correction . . . . . . . . . . . . . 127

4.17 .6 Electroweak Interaction . . . . . . . . . . . . . 128

5 Results 133

$5.1 \quad A_{1}+\eta A_{2} \ldots \ldots \ldots \ldots \ldots \ldots \ldots$

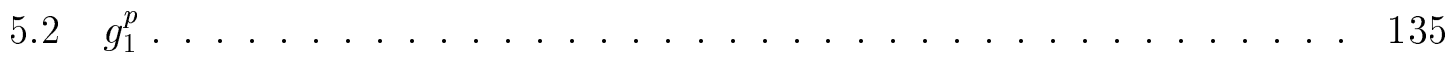

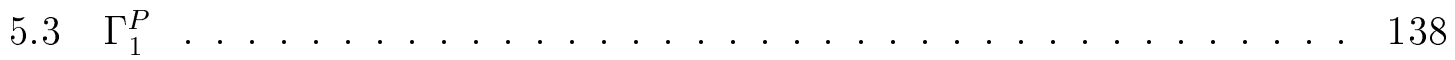

5.4 The Gerasimov, Drell and Hearn Sum Rule . . . . . . . . . . . . . . . 141

6 Conclusion $\quad 149$

$\begin{array}{ll}\text { A Error Analysis } & 151\end{array}$

B GDH derivation $\quad 156$

$\begin{array}{ll}\text { C Data Tables } & 160\end{array}$ 


\section{List of Figures}

2.1 Inclusive ep scattering $\ldots \ldots \ldots \ldots \ldots \ldots \ldots$

$2.2 \mathrm{~W}$ vs $Q^{2}$ for EG1 data $\ldots \ldots \ldots \ldots \ldots \ldots \ldots$

2.3 Compton Scattering $\ldots \ldots \ldots \ldots \ldots \ldots \ldots$

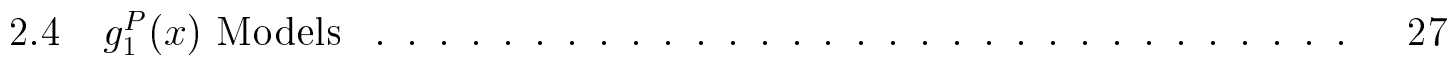

2.5 Vector Dominance Model Diagram _. . . . . . . . . . . . . . 29

$2.6 \quad \Gamma_{1}^{P}$ Models . . . . . . . . . . . . . . . . . . . . . . 32

3.1 The Thomas Jefferson National Accelerator Facility . . . . . . . . 35

3.2 The CLAS Detector . . . . . . . . . . . . . . . . 39

3.3 The Torus Magnet for CLAS . . . . . . . . . . . . . . 40

3.4 The CLAS drift chamber cells . . . . . . . . . . . . . 43

3.5 Time of Flight Counter . . . . . . . . . . . . . . . 44

3.6 Cerenkov Detector hardware . . . . . . . . . . . . . 46

$3.7 \mathrm{U}, \mathrm{V}$ and $\mathrm{W}$ layers of the Electrocalorimeter $\ldots \ldots \ldots \ldots$

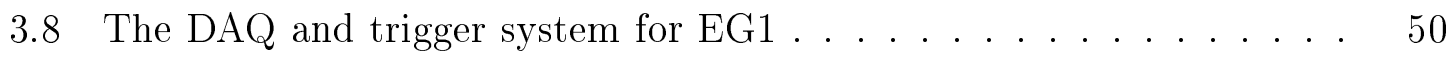

3.9 EG1 Polarized Target System . . . . . . . . . . . . . . . 53

3.10 Electron-Proton coupling diagram . . . . . . . . . . 56

3.11 Polarized target magnet and cryostat $\ldots \ldots \ldots \ldots \ldots \ldots$ 
3.12 EG1 evaporation refrigerator . . . . . . . . . . 66

3.13 Target insert . . . . . . . . . . . . . . . 69

3.14 Schematic of the Liverpool Q-meter and NMR system . . . . . . . 75

3.15 Extraction and integration of proton absorption signal . . . . . 78

4.1 RF Correction .......................... 85

4.2 Beam helicity and sync bit configuration . . . . . . . . . 87

4.3 Momentum Corrections - inbending . . . . . . . . . . . . 90

4.4 Momentum Corrections - outbending . . . . . . . . . . . . 90

4.5 Target and Cryostat Structure in the Beam . . . . . . . . . . 92

4.6 Vertex Correction . . . . . . . . . . . . . . 93

4.7 The $e^{+} / e^{-}$ratio as a function of $\breve{C}$ erenkov cut $\ldots \ldots . . \ldots 98$

$4.8 \mathrm{E} / \mathrm{p}$ cut $\ldots \ldots \ldots \ldots \ldots \ldots \ldots$

4.9 Quality Checks ...................... 100

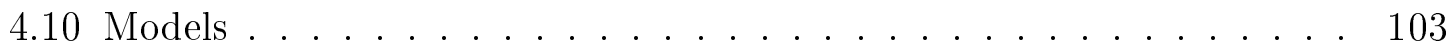

$4.11{ }^{15} \mathrm{~N}$ data . . . . . . . . . . . . . . . 106

4.12 Packing Fraction . . . . . . . . . . . . . . 108

4.13 Background Subtraction . . . . . . . . . . . . . 111

4.14 Beam and Target Polarization . . . . . . . . . . . . . 115

4.15 Positron/Electron Ratio . . . . . . . . . . . . . 117

4.16 Bremsstrahlung I . . . . . . . . . . . . . . . . . . . . . . 119

4.17 Bremsstrahlung II . . . . . . . . . . . . . . . . . . . . 119

4.18 Vertex Correction . . . . . . . . . . . . 120

4.19 Vacuum Polarization . . . . . . . . . . . . . 120

$4.20 g_{1}^{p}$ vs X for $Q^{2}=1.12 \mathrm{GeV}$ systematics . . . . . . . . . . 130 
$4.21 \Gamma_{1}^{P}$ systematics . . . . . . . . . . . . . . . 131

4.22 Generalized GDH systematics . . . . . . . . . . . . . . 132

$5.1 \quad A_{1}+\eta A_{2} \ldots \ldots \ldots \ldots \ldots \ldots$

5.2 Comparison of $A_{1}$ and $\eta A_{2} \ldots \ldots \ldots \ldots \ldots$

$5.3 Q^{2}$ evolution of $g_{1}^{p}$ vs. $\mathrm{x} \ldots \ldots \ldots \ldots \ldots$

$5.4 g_{1}^{p}$ vs. x for $Q^{2}=1.12 \mathrm{GeV}^{2} \ldots \ldots \ldots \ldots \ldots$

5.5 Resonance contribution to $\Gamma_{1}^{P} \ldots \ldots \ldots \ldots \ldots$

5.6 The First Moment of $g_{1}^{p} \ldots \ldots \ldots \ldots \ldots$. . . . . . . . . 144

5.7 Generalized GDH Sum Rule I . . . . . . . . . . . . . 146

5.8 Generalized GDH Sum Rule II . . . . . . . . . . . . . . . . 147 


\section{List of Tables}

1.1 Quark Quantum Numbers ... . . . . . . . . . . . . 2

2.1 Kinematic Variables in Lab Frame. . . . . . . . . . . . 8

3.1 EG1 Trigger Thresholds . . . . . . . . . . . . . . . . . . . . 49

3.2 Material in the Beamline . . . . . . . . . . . . . 64

4.1 Average Dilution Factor and Packing Fraction . . . . . . . . 110

4.2 Beam Polarization $\times$ Target Polarization . . . . . . . . . . . 114

4.3 Live-time Correction . . . . . . . . . . . . . . . . . . . 123

4.4 Systematic Error Summary . . . . . . . . . . . . . . . 125

$5.1 \quad$ EG1 Trigger Statistics . . . . . . . . . . . . . . . . . 134

5.2 Integration Limit in $\mathrm{W}$ for $Q^{2}$ bins $\ldots \ldots \ldots \ldots \ldots$

A.1 Faraday Cup Asymmetry Error . . . . . . . . . . . . . 153

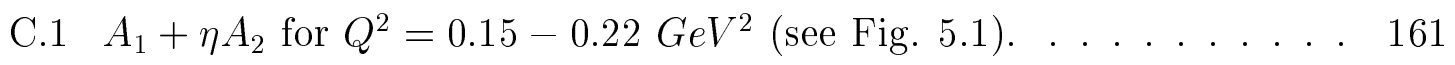

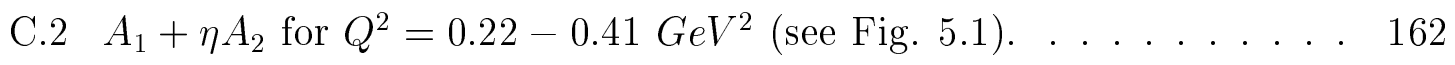

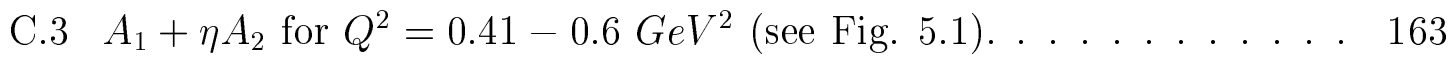

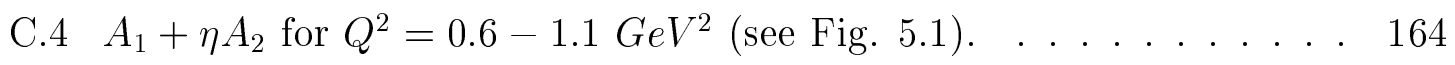


C.5 $g_{1}^{P}$ vs. $\mathrm{X}_{B J}$ for $Q^{2}=0.15-0.27 \mathrm{GeV}^{2}$ (see Fig. 5.3). . . . . . . . . 165

C.6 $g_{1}^{P}$ vs. $\mathrm{X}_{B J}$ for $Q^{2}=0.27-0.5 \mathrm{GeV}^{2}$ (see Fig. 5.3). . . . . . . . 166

C.7 $g_{1}^{P}$ vs. $\mathrm{X}_{B J}$ for $Q^{2}=0.5-0.74 \mathrm{GeV}^{2}$ (see Fig. 5.3). . . . . . . . . 167

C.8 $g_{1}^{P}$ vs. $\mathrm{X}_{B J}$ for $Q^{2}=0.74-1.10 \mathrm{GeV}^{2}$ (see Fig. 5.3). . . . . . . . 168

C.9 $g_{1}^{P}$ vs. $\mathrm{X}_{B J}$ for $Q^{2}=1.10-1.64 \mathrm{GeV}^{2}$ (see Fig. 5.3). . . . . . . . . 169

C.10 $\Gamma_{1}^{P}$ vs. $\mathrm{Q}^{2}$ integrated up to $\mathrm{W}=2 \mathrm{GeV}$ (see Fig. 5.5). . . . . . . 170

C.11 $\Gamma_{1}^{P}$ vs. $Q^{2}$ integrated over full $\mathrm{W}$ phase space available (see Fig. 5.6). 171

C.12 $\Gamma_{1}^{P}$ vs. $\mathrm{Q}^{2}$ integrated over full $\mathrm{W}$ phase space available with the deep inelastic contribution included (see Fig. 5.6) . . . . . . . . . . 172

C.13 $I_{1}$ vs. $\mathrm{Q}^{2}$ integrated over full $\mathrm{W}$ phase space available (see Fig. 5.7). . 173

C.14 $I_{1}$ vs. $\mathrm{Q}^{2}$ integrated over full $\mathrm{W}$ phase space available with DIS contributed included (see Fig. 5.7) . . . . . . . . . . . . . . 174 


\section{Chapter 1}

\section{The Quark Review}

The truth is always a compound of two half truths, and you never reach it, because there is always more to say.

\section{- Tom Stoppard}

In 1964 Gell-Mann [1] and Zweig [2], in order to explain the sudden proliferation of particles, proposed that the proton and other hadrons were composed of smaller and more elementary particles which Gell-Mann termed "quarks". In order to construct the known quantum numbers of hadrons, Gell-Mann and Zweig needed three species of quarks, up (u), down (d) and strange (s). As more particles were identified three additional quarks termed charm (c), truth (t) and beauty (b) were hypothesized. Baryon states, such as the proton and nucleon, were constructed from three quark (qqq) bound states and mesons from quark-anitquark ( $(\bar{q})$ pairs. The six different types of quarks are referred to as flavors and their properties and quantum numbers are summarized in Table 1.1[7]. 
Table 1.1: Quark Quantum Numbers

\begin{tabular}{|c|c|c|c|c|c|}
\hline flavor & charge & current mass & spin & baryon\# & isospin $\left(I_{3}\right)$ \\
\hline \hline $\mathrm{u}$ & $\frac{2}{3} e$ & $1-5 \mathrm{MeV}$ & $\frac{1}{2}$ & $\frac{1}{3}$ & $\frac{1}{2}\left(\frac{1}{2}\right)$ \\
\hline $\mathrm{d}$ & $-\frac{1}{3} e$ & $3-9 \mathrm{MeV}$ & $\frac{1}{2}$ & $\frac{1}{3}$ & $\frac{1}{2}\left(-\frac{1}{2}\right)$ \\
\hline $\mathrm{s}$ & $-\frac{1}{3} e$ & $75-170 \mathrm{MeV}$ & $\frac{1}{2}$ & $\frac{1}{3}$ & $0(0)$ \\
\hline $\mathrm{c}$ & $\frac{2}{3} e$ & $1.15-1.35 \mathrm{GeV}$ & $\frac{1}{2}$ & $\frac{1}{3}$ & $0(0)$ \\
\hline $\mathrm{b}$ & $-\frac{1}{3} e$ & $4.0-4.4 \mathrm{GeV}$ & $\frac{1}{2}$ & $\frac{1}{3}$ & $0(0)$ \\
\hline $\mathrm{t}$ & $\frac{2}{3} e$ & $\sim 170 \mathrm{GeV}$ & $\frac{1}{2}$ & $\frac{1}{3}$ & $0(0)$ \\
\hline
\end{tabular}

Quarks were expected to have a spin of $\frac{1}{2}$ and therefore required to follow Fermi-Dirac statistics. However one of the known nucleon bound states, the $\triangle^{++}$, is composed of three $u$ quarks in a state of zero angular momentum. This results in a wave function that is totally symmetric in spin, flavor and angular momentum. In order to ensure the anti-symmetrization of the ${\triangle^{++}}^{+}$wavefunction Han, Nambu, Greenberg and Gell-Mann [3][4][5] proposed an additional unobserved quantum number which they called color. They required all baryon states to be antisymmetric in color so that the overall wave function is properly antisymmetrized. There are three possible colors in the simplest model: red, green and blue. The role of color in the developing theory of Quantum Chromodynamics (QCD) wasn't fully realized until deep inelastic experiments at CERN and SLAC demonstrated that the strong coupling of partons has the property of asymptotic freedom. Once non-Abelian gauge theories were shown to describe this property it was natural to identify the color symmetry with the gauge group. Thus, QCD describes quarks as point particles, which come in six different flavors, and interact by exchanging color via a gauge boson known as the gluon.

The Quark Parton Model (QMP) describes nucleons that are composed of three 
valence quarks which have no orbital angular momentum and no polarized gluons. This model successfully describes the charge, color, isospin, parity and baryon numbers of the proton by simply adding up the relevant quantum numbers of the valence quarks. QPM does not describe the interactions within the proton, however, and therefore a more comprehensive theory is needed. The Operator Product Expansion (OPE)[12][13][14] was developed within the framework of QCD to describe more accurately the quark-gluon interactions within the proton. Implementation of the OPE, along with assumptions of SU(3) symmetry and an unpolarized strange sea, initially led to the prediction that the valence quarks would account for $100 \%$ of the proton spin. In the late 1980's a series of experiments [17][18][19][20] were carried out to measure the quark contribution to the spin of the proton. These experiments at SLAC and CERN found that, within this model, the quark contribution is quite small - less than $1 / 8$ of the total proton spin. This was surprising to the community and led to speculation about other possible carriers of spin such as the gluons and the orbital angular momentum of the quarks. All of these experiments took place in the deep inelastic scattering (DIS) region which is characterized by very energetic lepton beams and high momentum transfers $\left(Q^{2}\right)$ to the nucleon. When the proton is probed at large $Q^{2}$, the wavelength of the virtual photon is small enough that it interacts only with one quark. This quark is "free" in the sense that it is not interacting with any of the neighboring valence quarks during the time of the interaction. It also possesses momentum which is highly aligned with that of the nucleon, which in turn places the system in a symmetric ground state of angular momentum. In this region the deep inelastic proton scattering phenomena is reformulated in terms of elastic scattering off individual quarks which carry a fraction, $\mathrm{x}=\frac{Q^{2}}{2 M_{P} \nu}$, of the total 
proton momentum. Bjorken [6] predicted that the structure functions which describe the momentum and spin distributions of the proton, $F_{1}, F_{2}, g_{1}$ and $g_{2}$, would scale in the region of high momentum transfer. The functions no longer vary with $Q^{2}$, but instead depend only on $\mathrm{x}$. Scaling is a fundamental prediction because it requires that the quarks be structureless and non-interacting as $Q^{2} \rightarrow \infty$.

In reality there are scaling violations due to transverse quark momentum and quark-gluon interactions. As the momentum transfer becomes smaller, the wavelength of the virtual photon becomes larger, and there is a higher probability that the probe no longer interacts with only a single quark whose momentum is aligned with that of the nucleon. Eventually, as $Q^{2} \rightarrow 0$, approaching the real photon regime, the virtual photon is only sensitive to the static properties of the nucleon, such as the charge, magnetic moment and vector polarizabilities. For $Q^{2}$ values between these two extremes the virtual photon interacts with one or several parts of the coherent sum at a time.

It is difficult to calculate how the spin structure functions evolve as a function of $Q^{2}$ for several reasons. The first has to do with the breakdown of traditional perturbative methods in calculating the moments of $g_{1}$. In the scaling region perturbative QCD can be used to calculate the $Q^{2}$ dependence because the strong coupling constant, $\alpha_{s}$, is still small. However the validity of $\mathrm{pQCD}$ calculations for $Q^{2}$ of a few $\mathrm{GeV}$ is controversial. Other tools such as the operator product expansion have been used to predict higher twist contributions to the first moment of $g_{1}$ down to $Q^{2}=0.5 \mathrm{GeV}^{2}$. Lattice calculations show the most promise in developing predictions for the low $Q^{2}$ behavior of spin structure functions in the resonance region, but they are, as of yet, unrealized. 
The second source of difficulty in calculating the $Q^{2}$ evolution of the spin structure functions is due to the increasing contributions from the resonance region. The resonance region is defined as the regime where the invariant mass of the final state is less than $2 \mathrm{GeV}$. At low $Q^{2}$ the probability of the exchanged virtual photon exciting a nuclear transition is much greater and thus the resonance region becomes more important in describing nucleon spin behavior. Unfortunately resonance behavior is not well described by any of our current models and the data in this region is sparse. In general most predictions for the spin structure functions and their moments in the low $Q^{2}$, resonance region are not developed from rigorous theoretical constructs. Instead they are parameterized interpolations between the well tested Bjorken Sum Rule[6], which is only applicable in the scaling region, and the Gerasimov-Drell-Hearn Sum Rule [24][23], which is the only strong theoretical basis for predicting $\Gamma_{1}$ as $Q^{2} \rightarrow 0$. The GDH sum rule relates the difference in aligned and anti-aligned real photonproton cross-sections to the proton magnetic moment. The generalization of the GDH Sum Rule to virtual photon scattering then allows the asymptotic behavior of $\Gamma_{1}$ as it approaches the real photon point to be defined.

The lack of strong theoretical predictions and experimental data in the low $Q^{2}$ resonance region provided the impetus for running Jefferson Lab experiment 91-023 (EG1). The need to test the GDH sum rule (and the fundamental principles it rests upon), to refine knowledge of the resonance contributions to the structure functions, as well as to provide an experimental check for low $Q^{2}$ perturbative QCD calculations are a few of the primary motivations behind this type of experiment. Although not discussed in this analysis, EG1 data also have the capability of providing insight into quark-gluon interactions via the extraction of higher twist contributions as well as 
testing the validity of duality for spin structure functions. The results presented in this thesis will hopefully serve as a springboard to develop new models, and refine established ones, of spin structure functions in low $Q^{2}$ resonance region. 


\section{Chapter 2}

\section{Formalism}

\subsection{Electron-Proton Scattering Kinematics}

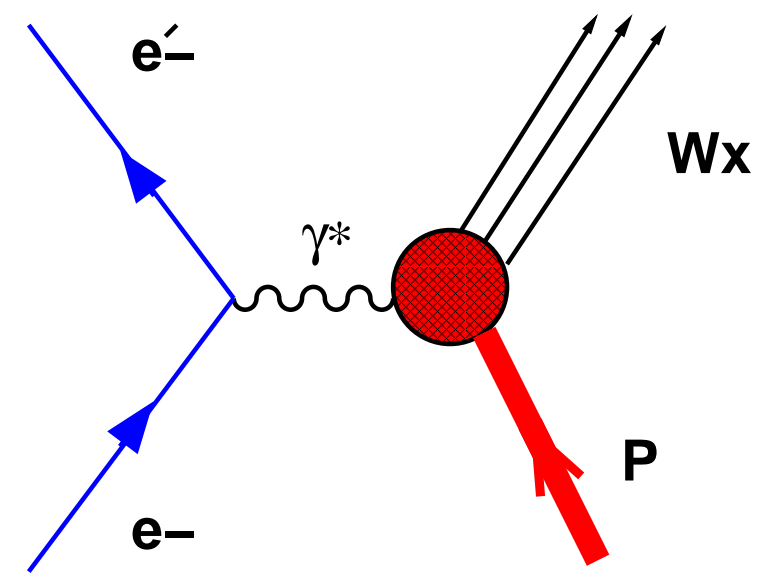

Figure 2.1: Inclusive electron-proton scattering

There are several probes that can be used to investigate the spin properties of the partons in the proton. The primary interaction studied in Jefferson Lab Experiment 
Table 2.1: Kinematic Variables in Lab Frame

\begin{tabular}{|c|c|l|}
\hline Variable & Expression & Meaning \\
\hline \hline$M_{p}$ & $M_{p}$ & Proton Rest Mass \\
\hline$m$ & $m$ & Electron Rest Mass \\
\hline$k^{\mu}$ & $(E, 0,0, \mathbf{k})$ & 4-momentum for initial $e^{-}$ \\
\hline$s^{\mu}$ & $\frac{1}{m}(\mathbf{k}, 0,0, E)$ & spin of initial $e^{-}$ \\
\hline$k^{\prime \mu}$ & $\left(E^{\prime}, \mathbf{k}^{\prime}\right)$ & 4-momentum of scattered $e^{-\prime}$ \\
\hline$\theta$ & $\theta$ & electron scattering angle in lab frame \\
\hline$p^{\mu}$ & $\left(M_{p}, 0,0,0\right)$ & initial 4-momentum of proton \\
\hline$S^{\mu}$ & $\frac{1}{M_{P}}\left(\mathbf{p}, 0,0, M_{p}\right)$ & initial spin of proton \\
\hline$q^{\mu}$ & $k^{\mu}-k^{\mu \prime}$ & 4-momentum of $\gamma^{*}$ \\
\hline$\nu$ & $E-E^{\prime}$ & $\gamma^{*}$ energy \\
\hline $\mathbf{q}$ & $\mathbf{k}-\mathbf{k}^{\prime}$ & 3-momentum of $\gamma^{*}$ \\
\hline$Q^{2}$ & $4 E E^{\prime} \sin ^{2} \theta / 2$ & 4-momentum squared of $\gamma^{*}$ \\
\hline$W^{2}$ & $M_{P}^{2}-Q^{2}+2 M_{P} \nu$ & invariant Mass of hadronic final state \\
\hline$g_{\mu \nu}$ & $x_{\mu}=g_{\mu \nu} x^{\nu}$ & metric tensor of Minkowski space \\
\hline
\end{tabular}

91-023 (EG1) was a polarized electron exchanging one polarized virtual photon with a polarized proton. Relativistic electrons are clean probes because they are point particles which interact via the well defined electro-weak force. Figure 2.1 shows the Feynman diagram for this interaction and Table 2.1 gives the notation used in the following sections to discuss this process.

The kinematic scope of EG1 encompasses the entire resonance region for $Q^{2}$ values of $0.15-2.0 \mathrm{GeV}^{2}$. The resonance region is so termed because of the characteristic 
peaks in the $\mathrm{W}$ spectrum which represent excited states of the nucleon. Although the resonance region is characterized by an invariant mass, W, less than $2 \mathrm{GeV}$, Fig. 2.2 demonstrates that the EG1 data extends up to regions of $3 \mathrm{GeV}$ in $\mathrm{W}$ for the higher beam energies.

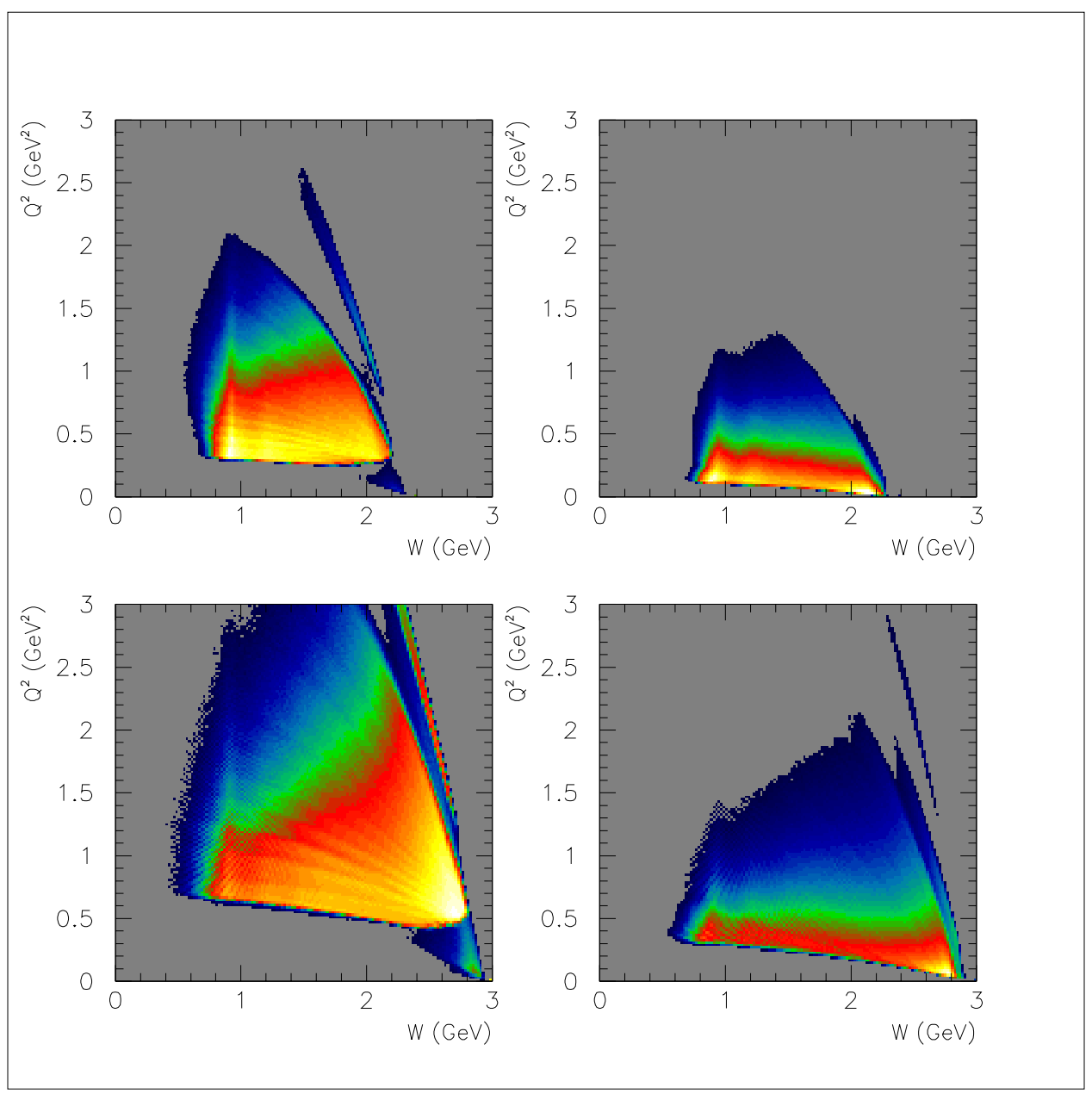

Figure 2.2: $\mathrm{Q}^{2}$ vs. Invariant Mass plots show kinematic range of the EG1 data. The top row shows $2.6 \mathrm{GeV}$ inbending and outbending torus settings and the bottom shows $4.3 \mathrm{GeV}$ inbending and outbending settings. Brighter colors translate to higher statistics 


\subsection{Electron-Nucleon Cross-Sections}

A comprehensive review of polarized and unpolarized scattering and structure function is given in An Introduction to Quarks and Leptons by Frank Close. The following sections will summarize the parts of this discussion that are immediately relevant to the EG1 experiment and analysis.

The electron-proton scattering cross section can be expressed in terms of the leptonic and hadronic currents, $L_{\mu \nu}$ and $W^{\mu \nu}$.

$$
\frac{\mathrm{d}^{2} \sigma}{\mathrm{d} \Omega \mathrm{d} E^{\prime}}=\frac{\alpha^{2}}{2 M_{P} Q^{4}} \frac{E^{\prime}}{E} L_{\mu \nu} W^{\mu \nu}
$$

The leptonic current contains information about the momentum and spin of the electron at the virtual-photon electron vertex and can be expressed explicitly as,

$$
L_{\mu \nu}\left(k, s ; k^{\prime}, s^{\prime}\right) \equiv\left[\bar{u}\left(k^{\prime}, s^{\prime}\right) \gamma_{\mu} u(k, s)\right]^{*}\left[\bar{u}\left(k^{\prime}, s^{\prime}\right) \gamma_{\nu} u(k, s)\right]
$$

Summing over the final electron spins produces

$$
L_{\mu \nu}=2\left(k_{\mu}^{\prime} k_{\nu}+k_{\nu}^{\prime} k_{\mu}-\left(k^{\prime} \cdot k-m^{2}\right) g_{\mu \nu}-i m \epsilon_{\mu \nu \rho \sigma} s^{\sigma} q^{\rho}\right)
$$

The hadronic current can be expressed similarly, with $\mathrm{X}$ representing all possible final states of the proton (elastic and inelastic).

$$
\begin{aligned}
W_{\mu \nu} \equiv & \frac{1}{4 \pi M_{P}} \sum_{N} \int \prod_{n=1}^{N}\left(\frac{d^{3} p_{n}^{\prime}}{2 E_{n}^{\prime}(2 \pi)^{3}}\right) \sum_{s_{n}}\left\langle p, s\left|\tilde{J_{\mu}^{\dagger}}\right| X\right\rangle \\
& \times\left\langle X\left|\tilde{J}_{\nu}\right| p, s\right\rangle(2 \pi)^{4} \delta^{4}\left(p+q-\sum_{n} p_{n}^{\prime}\right)
\end{aligned}
$$


The important difference between $W_{\mu \nu}$ and $L_{\mu \nu}$ lies in the fact that the proton is not a Dirac particle like the electron and thus the virtual photon-proton vertex cannot be described by the $\gamma^{\mu}$ matrixes. Instead the proton electromagnetic current, $J_{\mu}$ is used. This current is not well known for all kinematic regions, but it is constrained by invariance under time and parity reversal and Lorentz covariance. A clear analogy to the leptonic current exists for the proton in elastic scattering, where the final state is the same as the initial state and is represented by spinors, $u(p)$ and $\bar{u}\left(p^{\prime}\right)$.

$$
\begin{gathered}
J^{\mu}=e \bar{u}\left(p^{\prime}\right) \Gamma^{\mu} u(p) e^{i\left(p^{\prime}-p\right) \cdot x} \\
\Gamma^{\mu}=\mathcal{F}_{1}\left(q^{2}\right) \gamma^{\mu}+\frac{\kappa}{2 M_{P}} \mathcal{F}_{2}\left(q^{2}\right) i \sigma^{\mu \nu} q_{\nu}
\end{gathered}
$$

In this expression $\mathcal{F}_{1}$ and $\mathcal{F}_{2}$ are two functions that when combined linearly describe the electric and magnetic structure of the proton. These functions, called the Pauli and Dirac form factors, are a way of parameterizing the properties of a composite system like the proton. Equation 2.7 gives the complete expression for the hadronic current for inelastic scattering.

$$
\begin{aligned}
W_{\mu \nu}= & F_{1}\left(x, Q^{2}\right)\left(-g^{\mu \nu}+\frac{q^{\mu} q^{\nu}}{q^{2}}\right) \\
& +\frac{F_{2}(x, Q 2)}{p \cdot q}\left(p^{\mu}-\frac{p \cdot q}{q^{2}} q^{\mu}\right)\left(p^{\nu}-\frac{p \cdot q}{q^{2}} q^{\nu}\right) \\
& +i \frac{g_{1}\left(x, Q^{2}\right)}{p \cdot q} \epsilon^{\mu \nu \rho \sigma} S_{\sigma} q_{\rho} \\
& +i \frac{g_{2}\left(x, Q^{2}\right)}{(p \cdot q)^{2}} \epsilon^{\mu \nu \rho \sigma}\left[(p \cdot q) S_{\sigma}-(S \cdot q) p_{\sigma}\right] q_{\rho}
\end{aligned}
$$

Just as in the elastic case there are structure functions, $F_{1}, F_{2}, g_{1}$ and $g_{2}$, which 
contain information about the structure of the proton. Since this hadronic current describes inelastic scattering these functions can be interpreted in terms of the proton substructure, specifically the partons that compose the nucleon.

Both the leptonic and hadronic current can be decomposed into a symmetric and antisymmetric part. The symmetric terms are defined by their invariance under the exchange of $\mu$ and $\nu$. When the product of $L_{\mu \nu}$ and $W^{\mu \nu}$ is performed all symmetricantisymmetric cross terms go to zero, allowing for a simple decomposition of the crosssection into symmetric and antisymmetric terms as well. The symmetric term holds information on unpolarized scattering and the antisymmetric on polarized electron polarized proton scattering.

Isolation of the spin dependent part of the cross section, and therefore $g_{1}$ and $g_{2}$, is possible by taking cross section differences. If a longitudinally polarized electron beam is scattered from a longitudinally polarized proton target the difference in the spin aligned and anti-aligned cross-sections gives the following expression.

$$
\frac{\mathrm{d}^{2} \sigma^{\uparrow \Uparrow}}{\mathrm{d} \Omega \mathrm{d} E^{\prime}}-\frac{\mathrm{d}^{2} \sigma^{\downarrow \Uparrow}}{\mathrm{d} \Omega \mathrm{d} E^{\prime}}=\frac{4 \alpha^{2} E^{\prime}}{Q^{2} M_{P} E \nu}\left[\left(E+E^{\prime} \cos \theta\right) g_{1}\left(x, Q^{2}\right)-2 x M_{P} g_{2}\left(x, Q^{2}\right)\right]
$$

The single arrow corresponds to the spin direction of the electron and the double arrow refers to the proton spin. The target can also be polarized transversely with respect to the beam. The difference in right and left transverse polarization crosssections also isolates the spin structure information and allows for an independent measurement of $g_{1}$ and $g_{2}$.

$$
\frac{\mathrm{d}^{2} \sigma^{\uparrow \Leftarrow}}{\mathrm{d} \Omega \mathrm{d} E^{\prime}}-\frac{\mathrm{d}^{2} \sigma^{\uparrow \Rightarrow}}{\mathrm{d} \Omega \mathrm{d} E^{\prime}}=\frac{4 \alpha^{2} E^{\prime 2}}{Q^{2} E M_{P} \nu} \sin \theta\left(g_{1}\left(x, Q^{2}\right)+\frac{2 E}{\nu} g_{2}\left(x, Q^{2}\right)\right)
$$


It is possible to construct two asymmetries $A_{\|}$and $A_{\perp}$ from these cross-section differences,

$$
\begin{aligned}
& A_{\|} \equiv \frac{\frac{\mathrm{d}^{2} \sigma \uparrow \pi}{\mathrm{d} \Omega \mathrm{d} E^{\prime}}-\frac{\mathrm{d}^{2} \sigma \downarrow \pi}{\mathrm{d} \Omega \mathrm{d} E^{\prime}}}{\frac{\mathrm{d}^{2} \sigma^{\top} \Uparrow}{\mathrm{d} \Omega \mathrm{d} E^{\prime}}+\frac{\mathrm{d}^{2} \sigma \downarrow}{\mathrm{d} \Omega \mathrm{d} E^{\prime}}} \\
& A_{\perp} \equiv \frac{\frac{\mathrm{d}^{2} \sigma^{\uparrow}}{\mathrm{d} \Omega \mathrm{d} E^{\prime}}-\frac{\mathrm{d}^{2} \sigma^{\uparrow} \Rightarrow}{\mathrm{d} \Omega \mathrm{d} E^{\prime}}}{\frac{\mathrm{d}^{2} \sigma^{\uparrow}}{\mathrm{d} \Omega \mathrm{d} E^{\prime}}+\frac{\mathrm{d}^{2} \sigma^{\uparrow} \Rightarrow}{\mathrm{d} \Omega \mathrm{d} E^{\prime}}}
\end{aligned}
$$

Inserting the appropriate cross-section differences into Eq.2.10 and Eq.2.11 it is clear that these asymmetries are related to the proton structure functions. Expressing structure functions in terms of asymmetries instead of cross sections allows the acceptance of the detector to be disregarded. The acceptance from the numerator and denominator cancel leaving in essence an asymmetry of rates. $A_{\|}$can then be written as,

$$
A_{\|}\left(W, Q^{2}\right)=C_{1} \times \frac{N^{L \Uparrow}-N^{\uparrow \Uparrow}}{N^{\downarrow \Uparrow}+N^{\uparrow \Uparrow}-C_{2}}
$$

where $\mathrm{N}$ is the number of counts, normalized to the total incident charge, for a specific helicity of scattered electron. $C_{2}$ is subtracted from the denominator in order to correct for counts due to unpolarized target material. $C_{1}$ contains multiplicative corrections to $A_{\|}$and is defined as:

$$
C_{1}=\frac{R_{c} P_{c} N_{c}}{P_{b} P_{T}}
$$

$P_{b} P_{t}$ is the product of the beam and target polarization and corrects for the fact that the target and beam are not $100 \%$ polarized. $R_{c}$ is the radiative correction factor and $P_{c}$ the pair symmetric background correction. $N_{c}$ accounts for the small polarization of the ${ }^{15} \mathrm{~N}$ in the ammonia. 


\subsection{Virtual Photon Scattering}

The formulation of the polarized electron-proton cross-section from the hadronic and leptonic currents introduces $g_{1}\left(x, Q^{2}\right)$ and $g_{2}\left(x, Q^{2}\right)$, the spin structure functions of the proton, in a complete but general way. It is possible to reformulate this interaction in terms of virtual photo-absorption cross-sections, which reflect the underlying processes that are occurring at the virtual photon-proton vertex. The optical theorem allows these photo-absorption cross-sections to be related to the imaginary part of the forward Compton scattering amplitudes and then to the polarized and unpolarized structure functions derived in Section 2.1.

Compton scattering is defined as a real photon interacting with a nucleon and resulting in the emission of a real photon. Diagram 2.3 shows that this interaction can be extended to virtual photons as well. If either vertex in Fig 2.3 is isolated it is exactly the same as the virtual photon-proton vertex in Figure 2.1.

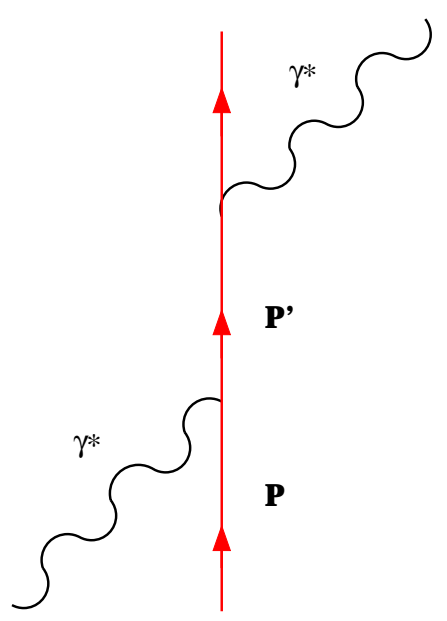

Figure 2.3: Compton Scattering

The optical theorem can be used to exploit this similarity. It states that the 
imaginary part of the Compton scattering amplitude at $\theta=0$ is proportional to the total Compton cross-sections, where $\mathrm{K}$ is the photon flux.

$$
\sigma_{\text {tot }}=\frac{4 \pi}{K} \operatorname{Im}(\mathcal{M}(\theta=0))
$$

Since the photo-absorption vertex and the virtual Compton scattering vertex are showing the same physics it is reasonable to use the optical theorem to state that the total photon absorption cross-section is proportional to the imaginary part of the forward Compton scattering amplitude. This allows all possible forward amplitudes to be calculated using standard QED calculation and trace methods and related to the virtual photon-proton vertex that is of interest.

In the case of virtual photon Compton scattering there are four independent forward helicity amplitudes, $\mathcal{M}_{\gamma_{i},\left(\gamma_{f}\right), p_{i},\left(p_{f}\right)}$. These amplitudes are constructed from all allowed combinations of photon and proton spin projection.

$$
\begin{aligned}
& \operatorname{Im}\left(\mathcal{M}_{+1,(+1),-\frac{1}{2},\left(-\frac{1}{2}\right)}\right) \equiv \sigma_{1 / 2}^{T}=\frac{4 \pi^{2} \alpha}{K M}\left(F_{1}+g_{1}-\frac{Q^{2}}{\nu^{2}} g_{2}\right) \\
& \operatorname{Im}\left(\mathcal{M}_{+1,(+1),+\frac{1}{2},\left(+\frac{1}{2}\right)}\right) \equiv \sigma_{3 / 2}^{T}=\frac{4 \pi^{2} \alpha}{K M}\left(F_{1}-g_{1}+\frac{Q^{2}}{\nu^{2}} g_{2}\right) \\
& \operatorname{Im}\left(\mathcal{M}_{+0,(+0),+\frac{1}{2},\left(+\frac{1}{2}\right)}\right) \equiv \sigma_{1 / 2}^{L}=\frac{4 \pi^{2} \alpha}{K M}\left(-F_{1}+\frac{1+Q^{2} / \nu^{2}}{2 x} F_{2}\right) \\
& \operatorname{Im}\left(\mathcal{M}_{+1,(+0),-\frac{1}{2},\left(+\frac{1}{2}\right)}\right) \equiv \sigma_{1 / 2}^{T L}=\frac{4 \pi^{2} \alpha \sqrt{Q^{2}}}{K M \nu^{2}}\left(g_{1}+g_{2}\right)
\end{aligned}
$$

$\mathrm{K}$ is defined as $\nu-\frac{Q^{2}}{2 M}$ and is the virtual photon flux. The subscripts of $\mathcal{M}$ refer to the initial (final) photon and proton helicities. The subscripts of $\sigma$ refer to the spin of the intermediate photon-proton spin state. The superscripts of the $\sigma$ refer to the transverse $(\mathrm{T})$ or longitudinal $(\mathrm{L})$ polarization of the virtual photon. It is important 
to underscore the fact that only the first two amplitudes would be possible with real photons since they by definition are massless and therefore only transverse.

It is clear that linear combinations of the above helicity amplitudes could isolate either the unpolarized or polarized spin structures of the proton. At this point it is useful to define the virtual photon-proton asymmetries $A_{1}$ and $A_{2}$.

$$
\begin{aligned}
& A_{1} \equiv \frac{\sigma_{1 / 2}^{T}-\sigma_{3 / 2}^{T}}{\sigma_{1 / 2}^{T}+\sigma_{3 / 2}^{T}}=\frac{g_{1}\left(x, Q^{2}\right)-\frac{Q^{2}}{\nu^{2}} g_{2}\left(x, Q^{2}\right)}{F_{1}\left(x, Q^{2}\right)} \\
& A_{2} \equiv \frac{\sigma_{1 / 2}^{T L}}{\sigma_{1 / 2}^{T}+\sigma_{3 / 2}^{T}}=\frac{\frac{Q}{\nu}\left[g_{1}\left(x, Q^{2}\right)+g_{2}\left(x, Q^{2}\right)\right]}{F_{1}\left(x, Q^{2}\right)}
\end{aligned}
$$

It is necessary to make clear that $A_{1}$ and $A_{2}$ are not directly measured in this experiment. However since $A_{1}$ and $A_{2}$ can be related to the experimental asymmetries $A_{\|}$and $A_{\perp}$ :

$$
\begin{aligned}
& A_{\|}=\mathcal{D}\left(A_{1}+\eta A_{2}\right) \\
& A_{\perp}=d\left(A_{2}-\zeta A_{1}\right)
\end{aligned}
$$

The kinematic variables $\mathcal{D}, d, \eta$ and $\zeta$ are defined as

$$
\begin{gathered}
\mathcal{D}=\frac{1-\epsilon \frac{E^{\prime}}{E}}{1+\epsilon R} \\
\eta=\frac{\epsilon \frac{\sqrt{Q^{2}}}{E}}{1-\epsilon \frac{E^{\prime}}{E}} \\
d=\mathcal{D} \sqrt{\frac{2 \epsilon}{1+\epsilon}} \\
\zeta=\eta \frac{1+\epsilon}{2 \epsilon}
\end{gathered}
$$




$$
\begin{gathered}
\epsilon=\frac{1}{\left(1+2 \tan \frac{\theta}{2}\left(1+\frac{Q^{2}}{\nu^{2}}\right)\right)} \\
R=\frac{\sigma_{L}}{\sigma_{T}}
\end{gathered}
$$

These equations relate the measurable quantities to the physics of virtual photonproton interactions. Ideally an experiment would be designed to measure both $A_{\|}$ and $A_{\perp}$, allowing an independent extraction of $A_{1}$ and $A_{2}$ and subsequent calculation of $g_{1}$ and $g_{2}$.

\subsection{Quark Distributions}

Sections 2.2 and 2.3 treated the proton as a single entity with spin of $\frac{1}{2}$ and charge $\mathrm{e}^{+}$. However it is now known that all baryons are made up of smaller, point-like Dirac particles called quarks. These quarks interact via the strong force, of which the spin 1 carrier is the gluon. This means that at high enough momentum transfer it should be possible to construct a cross-section that represents the elastic scattering of electrons off structureless point quarks inside of the proton. The unpolarized part of this cross-section would contain quark momentum distribution functions $q_{i}(x)$, which represent the probability that a quark of flavor $\mathrm{q}$ is carrying a fraction, $\mathrm{x}$, of the total nucleon momentum.

$$
q_{i}(x)=\left[q_{i} \uparrow+q_{i} \downarrow\right]+\left[\bar{q}_{i} \uparrow+\bar{q}_{i} \downarrow\right]
$$

The arrows indicate the helicity of the spin $\frac{1}{2}$ quarks and $\bar{q}_{i}$ is the antiquark contribution. Similarly the polarized part of the cross-section would contain the 
quark spin distribution functions, $\triangle q_{i}$

$$
\triangle q_{i} \equiv \int_{0}^{1} \delta q_{i}(x) d x
$$

where $\delta q_{i}(x)$ represents the difference between helicity states of a single quark flavor with momentum $\mathrm{x}$.

$$
\delta q_{i}(x)=\left[q_{i} \uparrow-q_{i} \downarrow\right]+\left[\bar{q}_{i} \uparrow-\bar{q}_{i} \downarrow\right]
$$

In the Quark Parton Model (QPM) these quark momentum and spin distributions are related to the unpolarized and polarized structure functions in the following way;

$$
\begin{aligned}
& F_{1}(x)=\frac{1}{2} \sum_{i} e_{i}^{2} q_{i}(x) \\
& F_{2}(x)=\sum_{i} e_{i}^{2} x q_{i}(x) \\
& g_{1}(x)=\frac{1}{2} \sum_{i} e_{i}^{2} \delta q_{i}(x) \\
& g_{2}(x)=0
\end{aligned}
$$

The QPM builds all the baryon ground states from free quarks of three flavors, up, down and strange. The proton is composed of two up, one down and no strange quarks. The neutron can be derived quickly from the proton state by exploiting isospin symmetry which allows all $\mathrm{u} \rightarrow \mathrm{d}$ and $\mathrm{d} \rightarrow \mathrm{u}$. QPM works well when predicting a few characteristics of the proton and neutron. For example the charge of the individual quarks adds up to the total proton and neutron charge of $1\left(2 \times \frac{2}{3}-\frac{1}{3}\right)$ and $0\left(2 \times \frac{1}{3}-\frac{2}{3}\right)$. Using Table 1.1 it is also clear that the QPM predicts other quantum numbers such as parity, baryon number and isospin. The QPM weakness lies in the fact that it 
does not describe quark-gluon interactions. In particular it assumes that only the valence quark spin, $\Delta \Sigma$, contributes to the overall spin of the proton. This requires that the gluon spin distribution, $\triangle \mathrm{G}$, and strange sea contribution, $\triangle s$, be zero. It also places the whole system (gluons, quarks and sea quarks) in the ground state of angular momentum. In summary the projection of the proton spin onto the z-axis is defined as:

$$
\begin{aligned}
S_{z} & =\frac{1}{2} \triangle \Sigma+\triangle G+L_{z} \\
& =\frac{1}{2}[\triangle u+\triangle d+\triangle s]+0+0 \\
& =\frac{1}{2}\left[\frac{4}{3}-\frac{1}{3}+0\right] \\
& =\frac{1}{2}
\end{aligned}
$$

The QPM predicts $\triangle \Sigma=1$. There are relativistic quark-parton models [9][10][11] which also assume $\triangle \mathrm{G}=0$ but allow for a non-zero angular momentum. These models predict a $\triangle \Sigma \approx 0.25$. It is clear that none of these models is complete and the much more comprehensive theory of Quantum Chromodynamics is needed to describe the interaction of the quarks and gluons inside the proton. The predictions of QCD will be discussed more thoroughly in the following section. 


\subsection{Sum Rules}

In order to test quark models experimentalists must have precise predictions of structure functions with which to compare their results. An elegant way to do this is to test a Sum Rule. Sum rules are integrals of a specific function, such as quark distributions or structure functions, over all $\mathrm{x}$. The model used to construct the function being integrated over determines what the result should be. For example, according to the quark-parton model an integral over the $\mathbf{u}$ valence quark distribution in the proton must be be equal to 2 .

$$
\int_{0}^{1} u_{v}(x) d x=2
$$

Similarly an integral over all strange quarks must equal zero.

$$
\int_{0}^{1}[s(x)-\bar{s}(x)] d x=0
$$

Integrals over structure functions are known as moments. The nth moment of an arbitrary structure function $\mathrm{f}(\mathrm{x})$ is

$$
\Gamma_{n} \equiv \int_{0}^{1} x^{n-1} f(x) d x
$$

Using the Quark Parton Model it is possible to calculate the first moment of $g_{1}(x)$.

$$
\begin{aligned}
\int_{0}^{1} g_{1}^{p}(x) d x & =\frac{1}{2} \sum_{i} e_{i}^{2} \triangle q_{i}(x) \\
& =\frac{1}{2}\left(\frac{4}{9} \triangle q_{u}+\frac{1}{9} \triangle q_{d}+\frac{1}{9} \triangle q_{s}\right)
\end{aligned}
$$


As discussed in the previous section, QCD accounts for the interactions of quarks and gluons and is capable of a more accurate prediction of sum rules such as the first moment of $g_{1}(x)$. Using the Operator Product Expansion [12][13][14] the first moment can be expressed at leading twist in terms of the singlet $\left(a_{0}\right)$ and non-singlet $\left(a_{3}\right.$ and $\left.a_{8}\right)$ proton matrix elements of the axial current.

$$
\Gamma_{1}^{p}=\int_{0}^{1} g_{1}^{p}\left(x, Q^{2}\right) d x=\left(\frac{1}{12} a_{3}+\frac{1}{36} a_{8}\right) C_{n s}+\frac{1}{9} a_{0} C_{s}
$$

The factors $C_{n s}$ and $C_{s}$ are the $Q^{2}$ dependent non-singlet and singlet QCD corrections. If $\triangle G$ is again assumed to be zero the proton matrix elements can be expressed in terms of quark helicities.

$$
\begin{aligned}
& a_{0}=\triangle u+\triangle d+\triangle s=\Delta \Sigma \\
& a_{3}=\triangle u-\triangle d=F+D \\
& a_{8}=\triangle u+\triangle d-2 \triangle s=3 F-D
\end{aligned}
$$

F and D are weak hyperon decay constants which can be extracted from data assuming $\mathrm{SU}(3)$ symmetry and have the values [15]:

$$
\begin{aligned}
F & =0.467 \pm 0.0079 D \\
& =0.8039 \pm 0.0080
\end{aligned}
$$

Assuming that the strange sea is unpolarized, $\triangle s=0$, and that the polarized gluon contribution, $\triangle G=0$, Ellis and Jaffe [16] predicted the first moment of $g_{1}^{p}(x)$. Their calculation results in $a_{0}=a_{8}=3 \mathrm{~F}-\mathrm{D}$ and the first moment can be expressed as 
follows:

$$
\int_{0}^{1} g_{1}^{p}(x) d x=\frac{1}{18}\left[C_{n s}(3 F+D)+2 C_{s}(3 F-D)\right]=0.1867
$$

A measurement of the first moment of $g_{1}(x)$ allows extraction of $a_{0}$ and subsequently the total z-projection of the quark spins. A focused experimental effort was given to isolating the first moment of $g_{1}$ in the deep inelastic region and thus isolating $a_{0}[17][18][19][20]$. The world - averaged outcome gives an $a_{0}=0.31 \pm 0.04$ [21]. The results of these measurements were unexpected for many reasons - the first being that they showed the Ellis-Jaffe Sum Rule to be violated. Even more surprising however was the fact that $a_{0}$ was much less than 1 , the value predicted by the Quark Parton Model. Clearly the majority of the spin of the proton is not carried by the valence quarks or a new definition of $a_{0}$ is required. Several schemes have been developed to incorporate a non-zero gluon polarization. The chiral invariant scheme[22] redefines $a_{0}$ as:

$$
a_{0}=\triangle \Sigma-\frac{3}{2 \pi} \alpha_{s}\left(Q^{2}\right) \triangle G\left(Q^{2}\right)
$$

In this definition it is still possible for the majority of the spin of the proton to be carried by the quarks as long as there is a large oppositely polarized gluon contribution.

A sum rule which is on much surer footing than the Ellis-Jaffe is the Bjorken Sum Rule [6]. Using current algebra, Bjorken derived a sum rule which relates the difference of the first moments of the proton and the neutron to the axial vector coupling constant.

$$
\Gamma_{1}^{p}-\Gamma_{1}^{n}=\frac{1}{6} \frac{g_{A}}{g_{v}} C_{n s}
$$

The Bjorken Sum Rule, modified to account for perturbative QCD corrections, 
has been verified within one standard deviation by many experiments. This may be due to the cancellation of all additional spin contributions such as the strange sea, gluon field and angular momentum when the proton and neutron subtraction is performed.

\subsection{Gerasimov-Drell-Hearn Sum Rule}

The sum rules discussed previously are valid in the deep inelastic scattering region. Historically this is the region that has received the most attention from theorists and experimentalists. The reason for this lopsided review is due to the fact that the strong coupling constant is very small at high $Q^{2}\left(>10 \mathrm{GeV}^{2}\right)$. This permits the quarks to be treated as essentially free particles and enables theorists to use familiar tools such as perturbation theory. As the momentum transfer of the photon becomes smaller, $\alpha_{s}$ approaches 1 these perturbative methods cannot be utilized. Presently only phenomological models that incorporate measured photo-production amplitudes are able to predict the low $Q^{2}$ evolution of spin structure functions. There is however a sum rule which describes the limiting behavior of the spin structure function $g_{1}^{p}$ as $Q^{2} \rightarrow 0$. It is called the Gerasimov-Drell-Hearn Sum Rule [23][24] and it relates the

difference in the $\sigma_{1 / 2}^{T}$ and $\sigma_{3 / 2}^{T}$ real photo-absorption cross-sections to the magnetic moment of the nucleon.

$$
\int_{\nu_{\text {thres }}}^{\infty} \frac{\sigma_{1 / 2}^{T}-\sigma_{3 / 2}^{T}}{\nu} d \nu=-\frac{2 \pi^{2} \alpha}{M^{2}} \kappa_{N}^{2}
$$

The photo-absorption cross-sections are related to the spin structure functions $g_{1}$ 
and $g_{2}$. This relation provides a limit on the behavior of $g_{1}$ as $Q^{2} \rightarrow 0$.

$$
\Gamma_{1}^{P}\left(Q^{2} \rightarrow 0\right) \Rightarrow \frac{Q^{2}}{16 \pi^{2} \alpha} \int_{\pi_{\text {thres }}}^{\infty}\left(\sigma_{1 / 2}^{T}(\nu)-\sigma_{3 / 2}^{T}(\nu)\right) \frac{d \nu}{\nu}=-\frac{Q^{2}}{8 M^{2}} \kappa_{N}^{2}
$$

The GDH sum rule requires that the first moment of $g_{1}$ goes to zero as $Q^{2}$ goes to zero with a negative slope. This means that $\Gamma_{1}^{P}$ is positive in the DIS region and then must cross zero and become negative before converging to zero at $Q^{2}=0$.

There exists a possibility that the GDH sum rule will be violated. Although the derivation is based on very general principles such as gauge invariance, crossing symmetry and unitarity, there is also the assumption of "the unsubtracted dispersion relation". If a non-zero constant is present then the GDH sum rule would become a "subtracted dispersion relation" and therefore ineffective in predicting the $Q^{2}$ evolution of the first moment. Preliminary results from real photon experiments [25] currently running at MAMI in Mainz, Germany, as well as at ELSA in Bonn, Germany, are consistent with the GDH Sum Rule expectations for the limited photon energies of 200-800 MeV. Future experiments are planned at SLAC, MAMI and ELSA in order to extend photon energies and further test the GDH Sum Rule. Despite some uncertainty, the generalized GDH Sum Rule remains the strongest theoretical predictor for the $Q^{2}$ evolution of the structure functions near the photon point.

\subsection{Low $Q^{2}$ Models for $g_{1}^{P}$ and The First Moment}

The Bjorken and Gerasimov-Drell-Hearn Sum Rules give the asymptotic behavior of $\Gamma_{1}^{P}$. One of the primary goals of this analysis however is to map out $g_{1}$ and the first moment in the region of transition from coherent hadronic scattering to incoherent 
elastic parton scattering. Several models and parameterizations have been employed in order to predict the $Q^{2}$ dependence of $g_{1}$ and the first moment of the proton (see Fig. 2.4 and 2.6). This section will introduce and discuss the primary principles behind each of these predictions.

\subsubsection{The $Q^{2}$ Evolution of $g_{1}^{P}$}

The $Q^{2}$ evolution of the spin structure functions has been of interest ever since scaling was predicted. However, even in the DIS regime it is known that $g_{1}$ does not scale perfectly. This is due to gluon emission and sea quark production. As the wavelength of the virtual photon becomes smaller it is able to resolve a quark after gluon emission, and therefore is only sensitive to the quark quantum numbers. A longer wavelength photon "sees" the quark emit and reabsorb a gluon and responds to the the properties of that combined system. Therefore from the beginning theoretical constructs were produced which would predict the behavior of polarized and unpolarized structure functions as they approached and entered the scaling region. As discussed previously, perturbative QCD and in particular the GLAP [30] equations have been able to reproduce very well experimental structure function measurements for $Q^{2}>3 \mathrm{GeV}^{2}$. It is natural that these methods would initially be used to extend these functions down to the low $Q^{2}$ values typical of the resonance region. Gehrmann and Stirling [31] calculated $g_{1}^{P}$ using leading and next-to-leading order pQCD theory. Using the QCD-improved model of $g_{1}^{P}$, the GLAP evolution equations and experimental data from SMC [18] and SLAC [21], a model for $g_{1}^{P}$ was produced for several $Q^{2}$ points , the lowest of which is $Q^{2}=1 \mathrm{GeV}^{2}$. The $Q^{2}=1$ curve is the only prediction that is within our regime and it is shown in Fig. 2.4. The fact that this model tries to use 
only deep inelastic tools to extend $g_{1}$ down to lower $Q^{2}$ results in a relatively smooth curve from the scaling region to $g_{1}^{P}=0$ at $\mathrm{x}=1$.

As it became clear that the low mass excitations of the proton seen in the resonance region contribute substantially to $\Gamma_{1}^{P}$, up to $20 \%$ at $Q^{2}=2 \mathrm{GeV}^{2}$ [33], models which include these resonance amplitudes were developed. Edelmann, Piller, Kaiser and Weise have presented a model which accounts for the dominant resonances and nonresonant background for $\mathrm{W}<1.7 \mathrm{GeV}^{2}$ and in addition incorporates existing data to parameterize $g_{1}^{P}$ for $W>1.7$. In this model $g_{1}$ was defined as:

$$
\left.g_{1}\left(x, Q^{2}\right)\right|_{R}=\frac{\nu M-Q^{2} / 2}{4 \pi^{2} \alpha} \frac{1}{1+Q^{2} / \nu^{2}}\left(\frac{\sigma_{1 / 2}^{T}-\sigma_{3 / 2}^{T}}{2}+\frac{Q}{\nu} \sigma_{1 / 2}^{L T}\right)
$$

Where R represents the $\triangle(1232), S_{11}(1535)$ and $D_{13}(1520)$, the only resonances accounted for in this model. The result, shown in Fig 2.4, is a curve at $Q^{2}=1 \mathrm{GeV}^{2}$ which shows significantly more structure than the Gehrmann and Stirling result. In particular $g_{1}^{P}$ is shown to be negative in the $\triangle$ region in $\mathrm{x}$, an artifact completely contributable to the inclusion of this resonance.

The parameterization developed by Edelmann et al. depends greatly on experimental data from electro and photo-production experiments. As the data on helicity amplitudes in the resonance region become more abundant and accurate these parameterizations can become more developed as well. A recent model by Simula, Osipenko, Ricco and Taiuti [34] incorporates all existing data on electro/photo-production in the resonance region, as well as the deep inelastic data to produce what is the most comprehensive prediction of $g_{1}^{P}$ in the resonance region to date. Curves from this model show significant structure throughout the high $\mathrm{x}$ region and will be compared rigorously to the results of this analysis. Plots of the model for $Q^{2}=1 \mathrm{GeV}^{2}$ are 


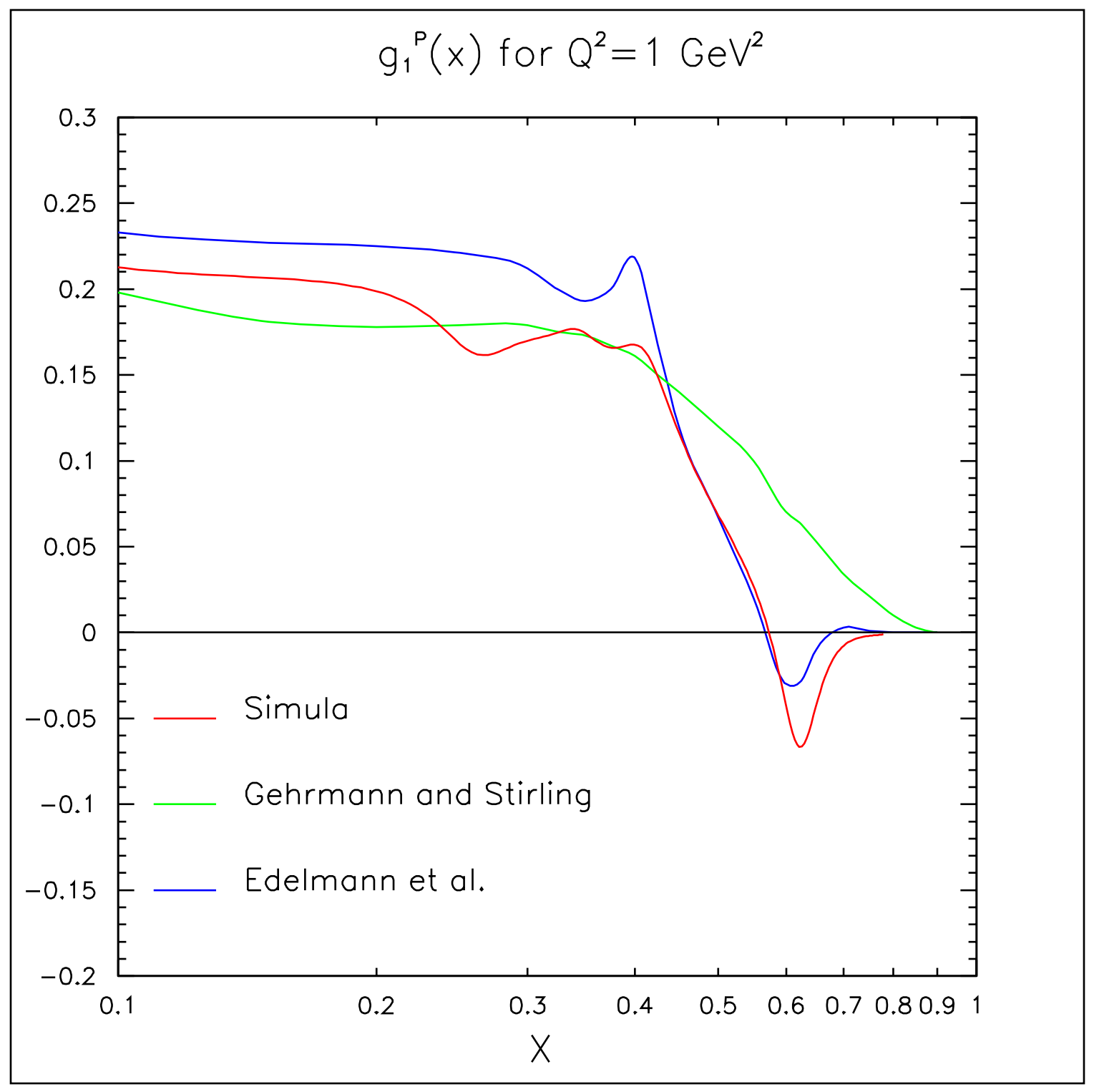

Figure 2.4: A plot of $g_{1}^{P}$ as a function of x for existing models. Inclusion of resonance amplitudes leads to significantly more structure in $g_{1}$, most noticeably in the $\triangle(1232)$ region. 
also shown in Fig. 2.4 for comparison with the Gehrmann and Stirling and Edelmann curves.

\subsubsection{The $Q^{2}$ Evolution of $\Gamma_{1}^{P}$}

At the same time lower $Q^{2}$ predictions for $g_{1}$ were emerging, the low $Q^{2}$ behavior of $\Gamma_{1}^{P}$ was also being explored. Anselmino, Ioffe and Leader[27] were the first to attempt to model $\Gamma_{1}^{P}$ in the resonance region. Using the GDH sum rule and newly published EMC[28] results as constraining parameters, the following form of the $Q^{2}$ dependence of the first moment was proposed.

$$
I\left(Q^{2}\right)=2 M_{P}^{2} \Gamma_{p}^{a s}\left[\frac{1}{Q^{2}+\mu^{2}}-\frac{c \mu^{2}}{\left(Q^{2}+\mu^{2}\right)^{2}}\right]
$$

where

$$
\begin{gathered}
I\left(Q^{2}\right)=\frac{2 M_{P}^{2}}{Q^{2}} \Gamma_{p}\left(Q^{2}\right) \\
\Gamma_{P}^{a s}=\Gamma_{p}(Q \rightarrow \infty) \\
c=1+\frac{1}{8}\left(\frac{\mu^{2}}{M_{P}^{2}}\right) \frac{\kappa^{2}}{\Gamma_{p}^{a s}}
\end{gathered}
$$

The variable $\mu$ is a mass parameter which characterizes the scale of the $Q^{2}$ variation. Allowing $\mu=m_{\rho}$ this form can be interpreted in terms of Vector Dominance Models (VDM), which were first introduced by Sakurai [35]. In the VDM the photonhadron interaction is characterized by a coupling of the photon to a vector meson, such as the $\rho, \omega$ or $\phi$ (see Fig.2.5). Time-like and space-like photons are always surrounded by a "hadronic cloud" which is composed of $q \bar{q}$ pairs. These pairs can form a vector meson which then interacts with the nucleon. The particular vector 
meson exchanged can be deduced by the decay products of the scattering reaction, allowing each of the observable nucleon resonances (such as the $\triangle(1232), S_{11}$ etc) to be calculated in terms of vector meson exchange amplitudes.

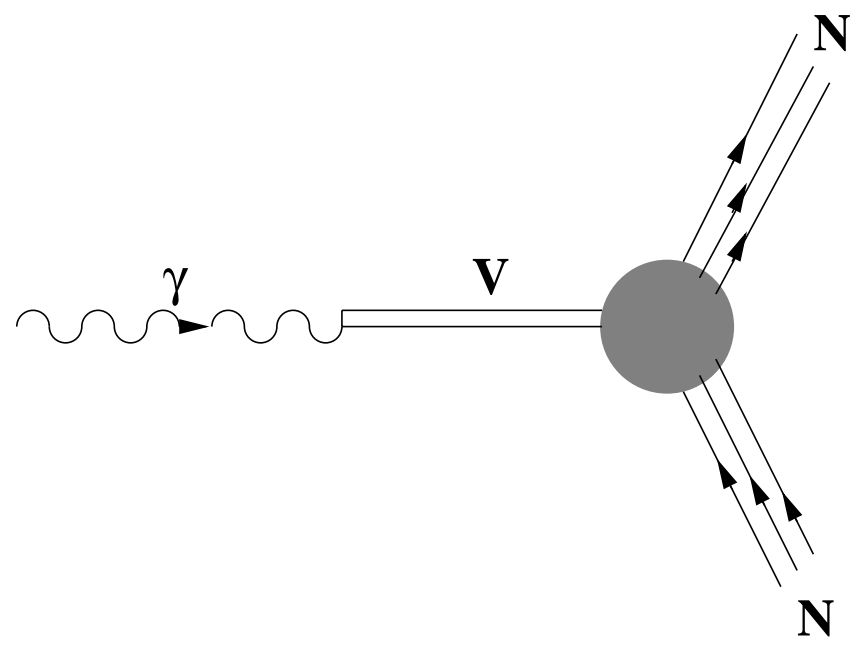

Figure 2.5: The Vector Dominance Model of photon-hadron interactions

In Eq. 2.55 the second term represents the diagrams in which a virtual photon interacts with the nucleon through vector mesons. The VDM predicts that this term will be negative at low $Q^{2}$. The first term represents the diagrams where one photon interacts via vector mesons and a second photon interacts directly with the hadron. This model produced a smooth curve for $\Gamma_{P}^{1}$ from the deep inelastic region of $\sim 0.2$, crossing zero at $Q^{2} \sim 1.0$ for $\mu=m_{\rho}$ and $Q^{2} \sim 2.5$ for $\mu=m_{\rho}$, and continuing down at a sharp slope and back up toward the GDH photon point prediction of zero at $Q^{2}=0$.

Burkert and Ioffe[36] improved this calculation by including the contribution of the low lying resonances, in particular the $\triangle(1232)$, to the electroproduction crosssection at small $Q^{2}$. This results in the following modification of eq.2.55: 


$$
I\left(Q^{2}\right)=\Gamma_{P}^{r e s}\left(Q^{2}\right)+2 M_{P}^{2} \Gamma_{p}^{a s}\left[\frac{1}{Q^{2}+\mu^{2}}-\frac{c \mu^{2}}{\left(Q^{2}+\mu^{2}\right)^{2}}\right]
$$

where $\Gamma_{P}^{r e s}\left(Q^{2}\right)$ is the contribution of all the baryonic resonances up to a $\mathrm{W}$ of 1.8 $\mathrm{GeVc}$ and $\mathrm{c}$ is now:

$$
c=1+\frac{1}{2} \frac{\mu^{2}}{M_{P}^{2}} \frac{1}{\Gamma_{p}^{a s}}\left[\frac{1}{4} \kappa_{p}^{2}+I_{P}^{r e s}(0)\right]
$$

Burkert and Ioffe argue that at the photon point $80 \%$ of the GDH integral is given by the $\triangle(1232)$ contribution. The inclusion of the highly negative $\triangle$, along with the most recent values of $\Gamma_{1}^{a s}$, produces (again with $\mu=m_{\rho, \omega}$ ) a smooth curve from the deep inelastic limit of $\sim 0.20$, crossing at a $Q^{2}=0.23$ and continuing sharply downward and back up toward zero.

Soffer and Teryaev[39] have also modeled the low $Q^{2}$ behavior of $\Gamma_{p}$. Their method exploits known information about the longitudinal-transverse interference cross-section which is related to the sum of $\Gamma_{1}+\Gamma_{2}$, where $\Gamma_{2}$ is the first moment of the structure function $g_{2}^{p}$. In the deep inelastic region the Burkhardt-Cottingham Sum Rule[72] requires that the contribution to $I\left(Q^{2}\right)_{1+2}=\frac{2 M^{2}}{Q^{2}}\left(\Gamma_{1}+\Gamma_{2}\right)$ comes solely from $\Gamma_{1}$, which is well known from previous experiments. At the photon point $I\left(Q^{2}\right)_{1+2}$ must converge to $\frac{\kappa_{P e_{P}}}{4}$. Since both the high and low $Q^{2}$ behavior are positive the authors are able to easily parameterize a smooth curve from the DIS region to $Q^{2}=0$ for $I_{1+2}$. In order to deduce the behavior of $\Gamma_{1}$ however $I_{2}$ must be subtracted from $I_{1+2}$. This is achieved by using a Schwinger Sum Rule for $I_{2}$, where $\mu$ is the nucleon magnetic moment and $G_{M}$ and $G_{E}$ the Sach's form factors.

$$
I_{2}\left(Q^{2}\right)=\frac{1}{4} \mu G_{M}\left(Q^{2}\right)\left[\mu G_{M}\left(Q^{2}\right)-G_{E}\left(Q^{2}\right)\right]
$$


It is trivial then to deduce the first moment of $g_{1}$ from $I_{1}=I_{1+2}-I_{2}$. The resulting model gives a zero $Q^{2}$ crossing of $0.2 \mathrm{GeV}$. This crossing is believed to be very stable and not strongly dependent on the parameterization of the $I_{1+2}$ due to the extremely strong $Q^{2}$ dependence of $I_{2}$.

The models described above predict the $Q^{2}$ behavior of $\Gamma_{1}^{P}$ integrated over the total inelastic mass spectra. However several models exist for limited $\mathrm{W}$ and $Q^{2}$ values in the resonance region. Two models exist which predict only the resonance ( $W \leq 2 \mathrm{GeV}$ ) contribution to the first moment, AO and MAID2000. AO [40] uses Born terms to produce a resonant and non-resonant background. Experimental data on helicity amplitudes are incorporated along with simple quark models in order to predict the $Q^{2}$ evolution of the virtual photon cross-sections $\sigma_{\frac{1}{2}, \frac{3}{2}}$. These crosssections are then used to calculate $\Gamma_{1}$ in the resonance region. Maid2000 [41] is a gauge-invariant, unitary model for one-pion photo and electro-production. The nonresonant background is calculated by evaluating the Feynman diagrams for the standard Born terms with mixed pseudovector-pseudoscalar $\pi N N$ coupling and vector meson exchange. The resonant contributions are based on the isobar model [42][43] and are assumed to have a Breit-Wigner energy dependence. The final scattering amplitude is unitarized using existing pion-nucleon, photo and electro-production data.

Finally, Ji and Osborne[37] have suggested that higher twist calculations, such as the one by Larin and Vermaseren [38], using the Operator Product Expansion should be used to predict $g_{1}^{p}\left(x, Q^{2}\right)$ from the DIS region down to a $Q^{2}=0.5 \mathrm{GeV}$. Working from $Q^{2}=0 \mathrm{GeV}^{2}$, Ji has used chiral perturbation theory to evolve the GDH sum rule to $Q^{2}$ of $0.2 \mathrm{GeV}^{2}$. Ji also calculates the total first moment including the elastic 


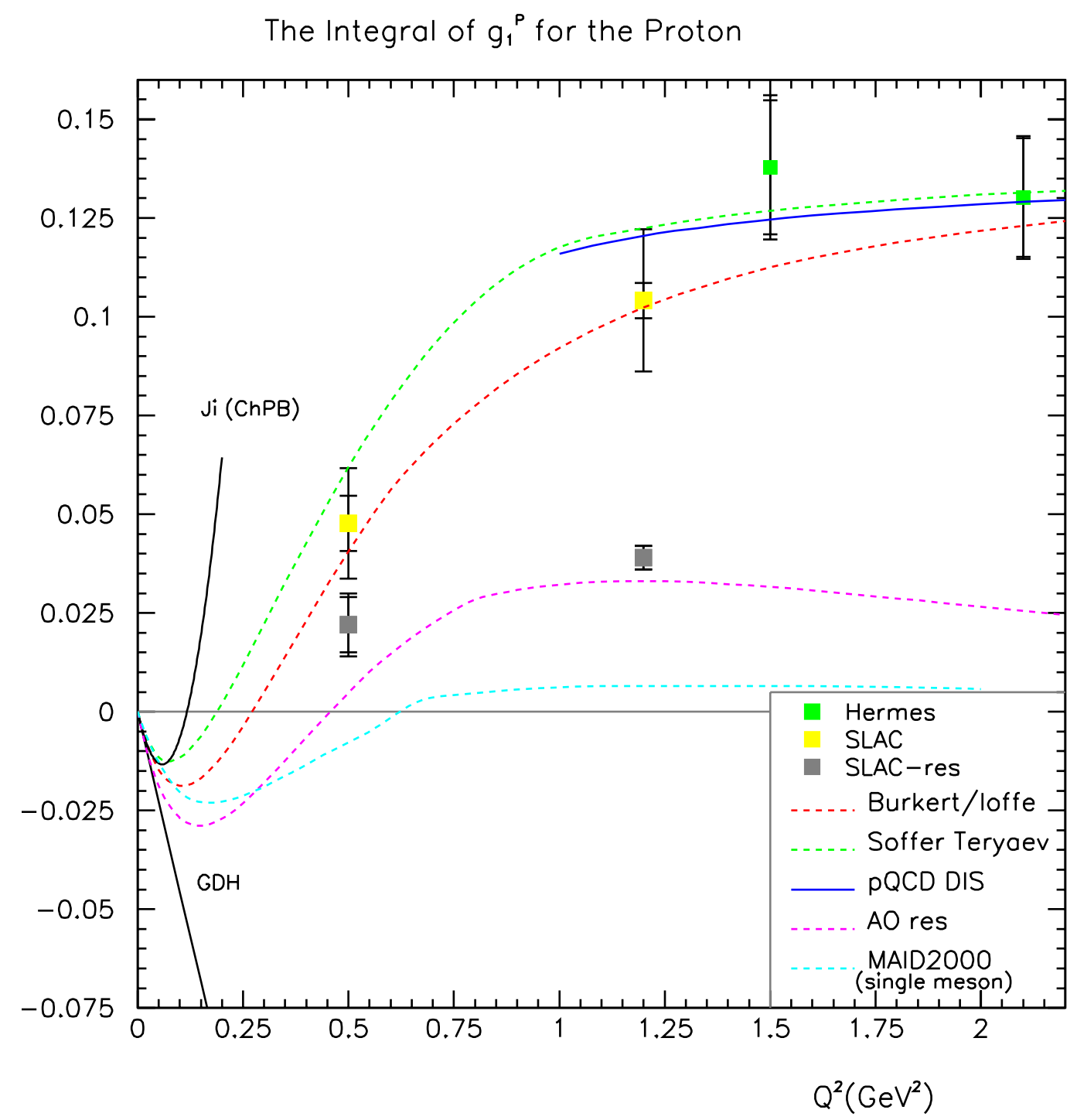

Figure 2.6: The plot above shows the low $Q^{2}$ pre-EG1 data for $\Gamma_{1}^{P}[21][29]$, as well as the various models described in section 2.72 . 
contribution to the integral. These predictions will not be compared with results presented in this thesis due to the fact that the measured elastic peak is used for normalization purposes and therefore cannot be included in the integral of the first moment. 


\section{Chapter 3}

\section{Experimental Setup}

Experiment 91-023, was one of four experiments in the EG1 run group that took place in the fall of 1998 at the Thomas Jefferson National Accelerator Facility (TJNAF) in Newport News, Virginia. TJNAF is equipped with three experimental halls, A, $\mathrm{B}$ and $\mathrm{C}$; each designed for different but complementary studies on the electromagnetic structure of the nucleon. The EG1 run took place in Hall-B, which houses the CEBAF Large Angle Spectrometer (CLAS). EG1 was the first experimental group to install and run with a dynamically polarized solid target inside of CLAS. This polarized target, optimized to fit the large acceptance of CLAS, allowed for the spin dependent electromagnetic structure of the proton and deuteron to be explored in the resonance region. The accelerator provided the low current, longitudinally polarized electron beam. The Polarized Electron Beam, CLAS, and the Polarized Target will be discussed in detail in the following sections. 


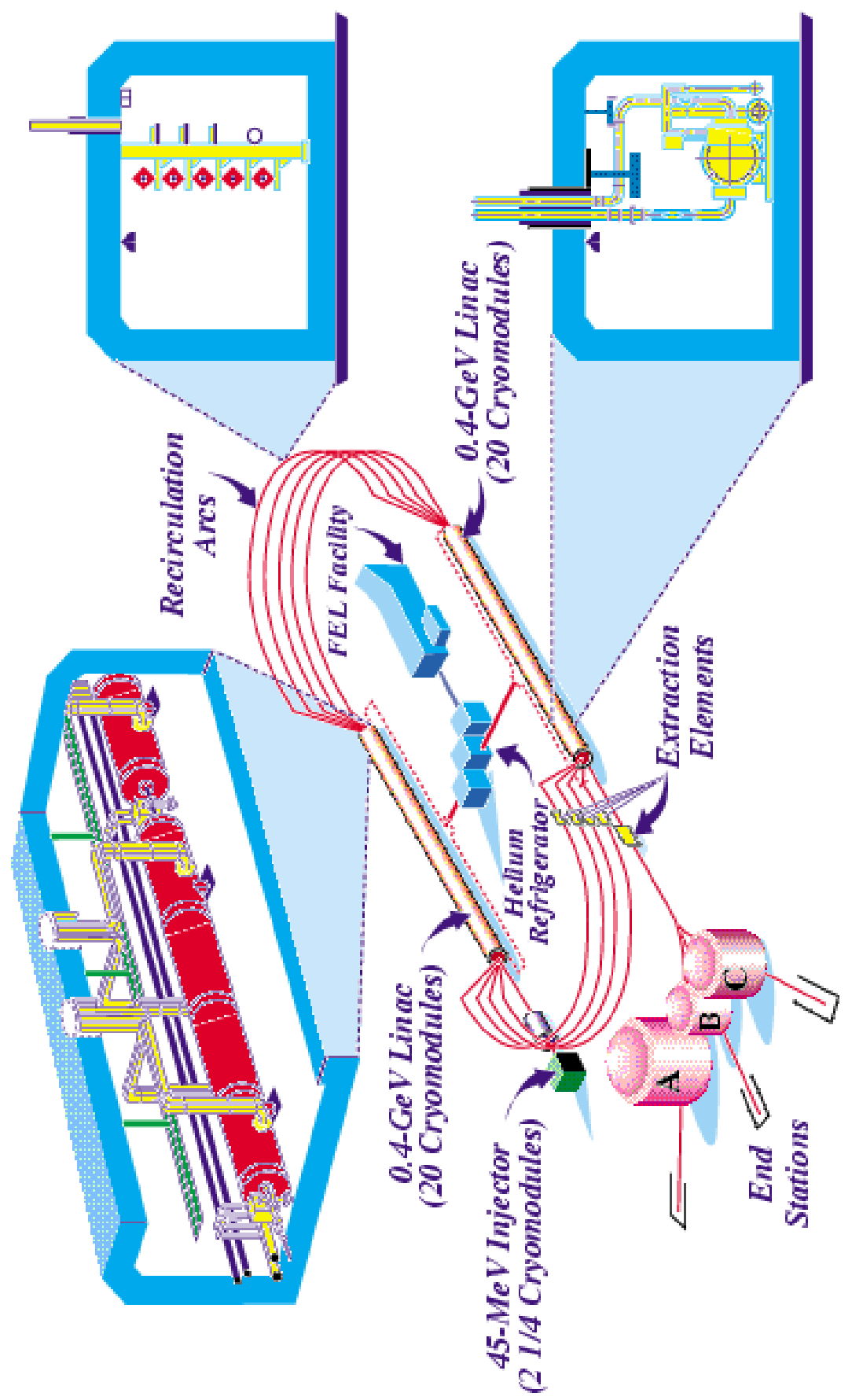

Figure 3.1: The Thomas Jefferson National Accelerator Facility 


\subsection{The Accelerator and Polarized Electron Beam}

The layout of the Thomas Jefferson Accelerator Facility is shown in Fig 3.1. The accelerator is composed of a $45 \mathrm{MeV}$ injector and two anti-parallel $0.5 \mathrm{GeV}$ linacs connected by five sets of recirculation arcs, giving the beamline a racetrack configuration. The linacs are composed of 20 cryomodules, each containing eight five-cell niobium cavities [45]. These superconducting radio-frequency $(\mathrm{RF})$ cavities operate in continuous wave $(\mathrm{CW})$ mode and have a minimum gradient of $5 \mathrm{MeV}$ per meter. At the time of EG1 running this resulted in a $\sim 800 \mathrm{MeV}$ boost to the beam for each pass around the racetrack. Recent improvements have pushed this boost capacity to $\sim 1000 \mathrm{MeV}$ per pass, allowing for a maximum beam energy of $5.7 \mathrm{GeV}$. Beams can be extracted at each recirculation, which allows polarized beams of varying energy and current to be delivered simultaneously to each of the three experimental halls. The energy spread of the beam is approximately $\triangle \mathrm{E} / \mathrm{E} \sim 10^{-4}$ with a current range of $100 \mathrm{pA}$ to $200 \mu \mathrm{A}$.

The polarized electron source consists of a laser, Pockels cell and a strained Gallium Arsenide (GaAs) crystal. Linearly polarized laser light is passed through the Pockels cell, an electro-optical crystal which converts the polarization from linear to circular. The voltage applied to the Pockels cell determines the helicity of the light and allows for the rapid pseudo-random switching of the electron beam polarization. The circularly polarized light is directed onto the GaAs photocathode [44], inducing electron transitions from the valence band to the conduction band. The frequency of light is chosen to preferentially pump specific electron spin transitions, resulting in a net polarization in the conduction band. A monolayer of cesium and fluorine applied to the surface of the crystal produces a vacuum energy level outside of the material 
which is less than the energy minimum of the conduction band. The electrons are released from the conduction band and accelerated by a $100 \mathrm{kV}$ cathode and injected into one of the linacs with an injection energy of approximately $45 \mathrm{MeV}$.

The orientation of the electron spin at injection is controlled by the angle of a Wien filter [47]. The Wien Filter consists of crossed electric and magnetic fields which are transverse to the particle motion. The electric field is defined by the desired spin rotation and the magnetic field is adjusted correspondingly so that the net Lorentz force on the particle is zero. This allows the angle of beam polarization with respect to the momentum to be changed without changing the direction or magnitude of the momentum. The resulting polarization is measured, before injection into the linac, using a $5 \mathrm{MeV}$ Mott polarimeter [46]. In Mott scattering a transversely polarized electron is scattered from the Coulomb field of a high Z nucleus (TJNAF used a gold target). Due to spin-orbit coupling there is a left-right scattering asymmetry which can be related to the total spin of the electron beam.

The beam is guided into the Hall-B beamline via the switch yard, which controls beam access into each of the three Halls. Once in the Hall-B beamline it is possible to control (raster) the horizontal and vertical alignment of the beam using the Beam Scan Controller (BSC). In EG1 the beam was rastered in a spiral pattern with diameter of $1-1.2 \mathrm{~cm}$. and a variable period of $1-10$ seconds. The beam polarization is again measured by a Møller Polarimeter [48] which is located upstream of the polarized target. The polarimeter is constructed from two iron-alloy foils which can be polarized parallel or anti-parallel to the spin of the incoming beam. Two quadrupole magnets guide the scattered electrons to two scintillating fiber detectors located symmetrically on the two sides of the beamline. By measuring the asymmetry between aligned and 
anti-aligned incoming electrons it is possible to extract the beam polarization. This polarization was monitored during the EG1 run to ensure that the beam polarization was high and consistent. The Møller Polarimeter measurements were not used in the data analysis because the beam and target polarization values were extracted from the elastic peak. The average beam polarization for the EG1 run was $~ 70 \%$.

Finally the beam current was monitored in Hall-B using an ungated Faraday Cup [49]. The Faraday cup is a block of lead which is located in the beam dump. Attached to the block is a capacitor which fills and discharges after $10^{-10}$ Coulombs has deposited on the block. The number of times the Faraday Cup capacitor discharged was recorded and used to monitor the beam current as well as the beam charge asymmetry, which was found to be $0.3 \%$ on average during the EG1 run.

\subsection{CLAS detector}

The CEBAF Large Acceptance Spectrometer was designed for experiments which need to detect several particles in the final state (see Fig 3.2). The acceptance is nearly $100 \%$ in the azimuthal angle and covers up to $140^{\circ}$ in the electron scattering plane. The momentum resolution, $\triangle p / p$, ranges from $0.5-1.0 \%$, depending on the momentum and scattering angle of the particle. These features allow a wide mass spectra, which includes all the known nucleon resonances, to be detected for a broad and continuous range of $Q^{2}$. Although multi-particle final states are not detected for the inclusive proton analysis, the continuous $Q^{2}$ acceptance of CLAS is ideal for examining the $Q^{2}$ behavior of the proton asymmetries and structure functions in the resonance region. 


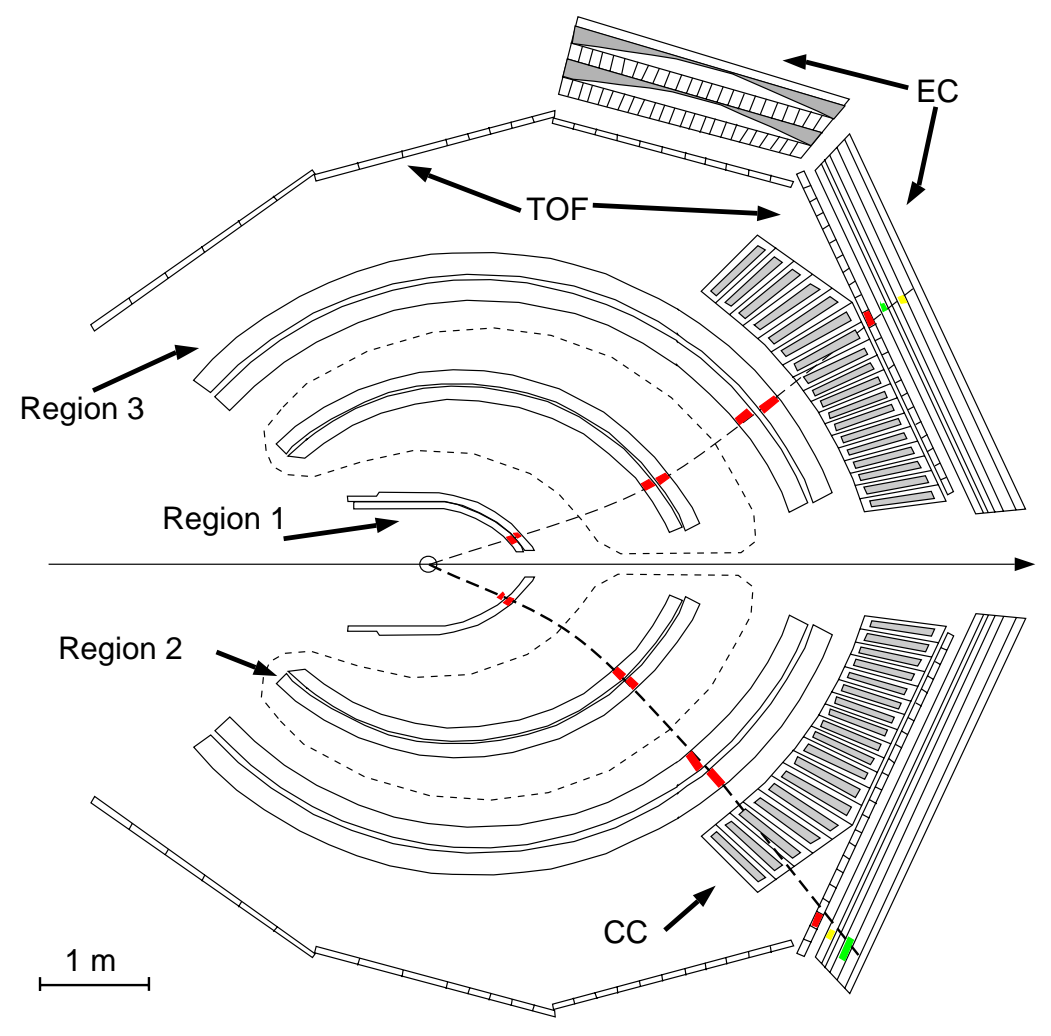

Figure 3.2: The CLAS Detector

The CLAS detector is divided into six sectors. The sectors cluster around the beampipe in such a way that the first sector is defined by azimuthal angles -30 to $+30^{\circ}$, where downstream is the positive $\mathrm{z}$ axis and positive $\mathrm{x}$ on the left. Between the six sectors are six superconducting coils, which together produce a toroidal field. Each sector contains three sets of Drift Chambers (DC), a $\breve{C}$ erenkov detector (CC), Time of Flight scintillators (TOF) and an Electromagnetic Calorimeter (EC). Sectors 1 and 2 also have a Large Angle Calorimeter (LAC). The drift chambers allow for tracking and subsequent particle momentum determination. The time of flight, $\breve{C}$ erenkov and calorimeters are used for particle identification and to determine the kinematics of the scattering event. CLAS provides a resolution sufficient for identification of electrons, 
pions, kaons, protons as well as deuterons. In addition the calorimeters can be used to detect photons and neutrons. Each of these detector packages will be discussed in detail in the following sections.

\subsubsection{Torus Magnet}

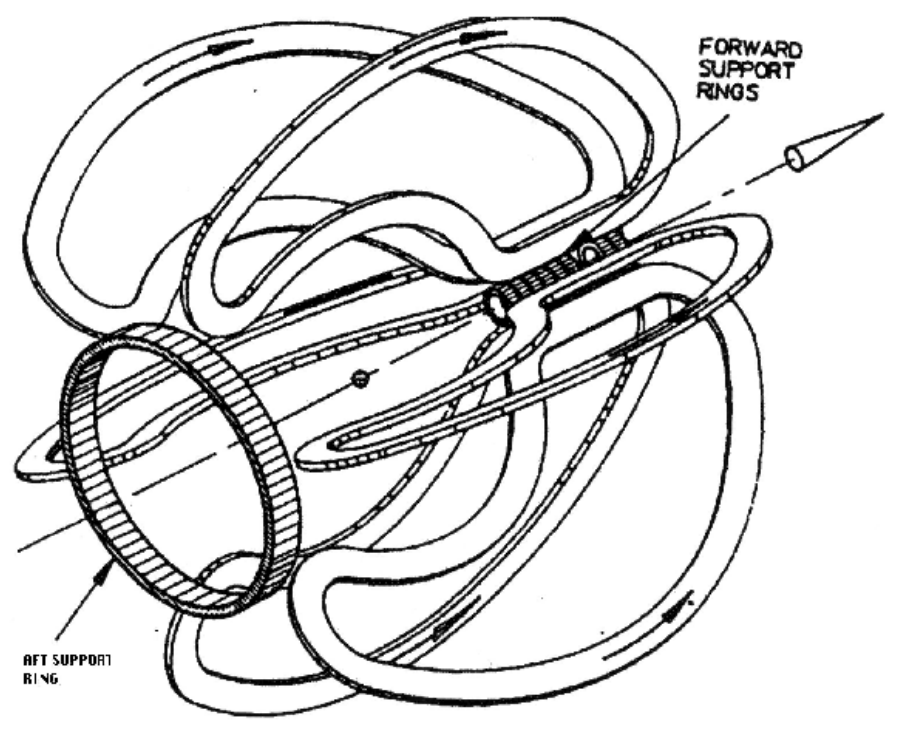

Figure 3.3: The Torus Magnet for CLAS

The torus magnet, designed for operation as part of the CLAS detector, was constructed and installed by Oxford Instruments. It is composed of six individual superconducting magnetic coils, located between each of the six sectors which hold the detector packages. It provides a magnetic field with a large transverse component to the particle momentum, allowing for high momentum resolution[50]. The field varies with polar angle, $\theta$, and ranges from $2 \mathrm{~T}$ at forward angles to $0.4 \mathrm{~T}$ at larger angles. The toroidal field preserves the azimuthal dependence of the event, resulting 
in an independent determination of $\phi$ and the momentum or scattering angle $\theta$. In practice this has the advantage of keeping the tracks of particles with different $\phi$ from mixing, with the disadvantage of making the reconstruction of these tracks more complicated and difficult because the field is inhomogeneous as well as losing a fraction of the total phi acceptance. The toroidal field also has the advantage of providing a field free region along the beamline.

\subsubsection{Drift Chambers}

Three sets of drift chambers are used to track the path, and therefore determine the momentum, of all charged scattered particles. Momentum and angle accuracy of $\triangle p / p \leq 0.5 \%$ and $\triangle \theta, \triangle \phi \leq 2$ mrad resulted in tracks measured to an accuracy of $100 \mu \mathrm{m}$ in the bend plane of the magnetic field and $1 \mathrm{~mm}$ perpendicular to the bend plane. An upper limit of $1 \%$ of a radiation length was also required in order to reduce multiple scattering events. A cursory description of the general construction and operation principles of the drift chambers is reviewed in this section. A complete discussion is given the The CLAS Drift Chamber System paper[51].

The first drift chamber set, region 1 (R1), is located in a low magnetic field region and close to the target. The region 2 (R2) chambers are larger and reside in the high magnetic field region between the coils of the torus. Region 3 (R3) are the largest set of chambers and are located outside of the torus magnet. Each region is divided into six sectors, all aligned with the R2 DC chambers which are defined and restricted by the torus coils.

The drift chambers operate on the principle that a charged particle traveling through a gas will ionize the atoms, thus creating positive and negative currents if 
placed within an electric field. Wires within the gas collect these negative ions and the resulting currents provide information on the path of the charged particle. The resolution of the track therefore is completely dependent on the position and number of the wires within each chamber.

Each sector of each region of drift chambers is supported by two endplates which are tilted at $60^{\circ}$ with respect to each other. Layers of positively biased sense wires and negatively biased field wires are strung from one endplate to the other in order to form hexagonal drift cells, Fig. 3.4. The wire direction is approximately perpendicular to the bend plane of the curved trajectories of the particles, maximizing sensitivity to the track momenta. Each of the three regions are divided into 2 superlayers, the first layer (axial) is perpendicular to the beam, and the second layer (stereo) is tilted at a $6^{\circ}$ angle to the axial wires. Each superlayer contains six layers of drift cells, except for the first superlayer of R1 which has only 4 layers of drift cells due to space limitations. A layer of guard wires surrounds the perimeter of each superlayer with the high-voltage potential adjusted to reproduce the electric field configuration given by an infinite grid of hexagonal cells. The drift chamber gas mixture, $90 \%$ argon- $10 \%$ $\mathrm{CO}_{2}$, was chosen to allow for reasonably low multiple scattering and short collection times.

\subsubsection{Time of Flight Detector}

The time of flight detector consists of a wall of scintillators outside of the region 3 drift chambers and oriented perpendicular to the track of the scattered particles. The main purpose of the TOF is to determine the time it takes for a particle to travel from the target to the scintillators. This information, along with the momentum from the DC, 


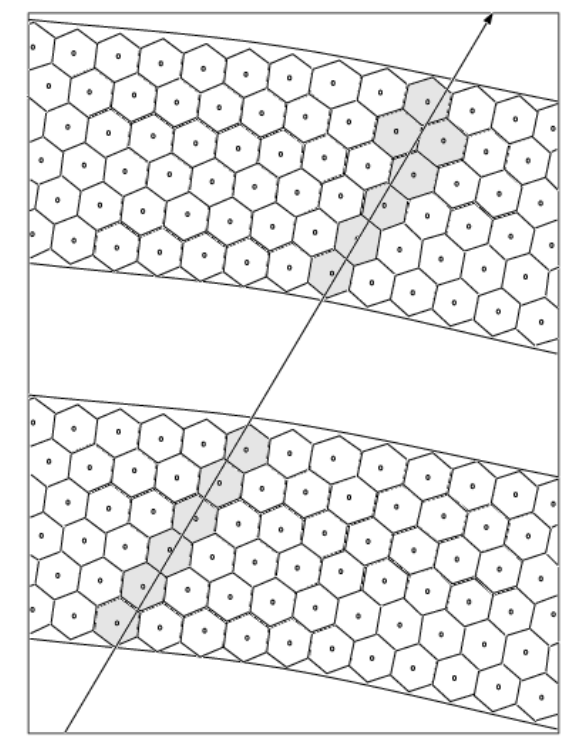

Figure 3.4: The three regions of CLAS each contain two superlayers of hexagonal drift cells. The sense wires form the centers and the field wires surrounding the centers give the hexagonal shape. Each layer is six cells deep.

aids in particle identification in offline analysis and allows more accurate triggering online. The system specifications required a time resolution of $120 \mathrm{ps}$ at the smallest angles and up to $250 \mathrm{ps}$ for angles greater than $90^{\circ}$. This design facilitates the separation of pion and kaons up to $2 \mathrm{GeV} / \mathrm{c}$ in momentum. A complete discussion of the construction and performance of the time of flight detectors is given in the paper The Time of Flight Detector for CLAS[52].

The TOF detector consists of 288 Bicron BC-408 scintillator strips, each of which is connected to two photomultiplier tubes. Each strip is $5 \mathrm{~cm}$ thick and has an angular coverage of approximately $2^{\circ}$ each. The time and pulse height are measured for each photomultiplier tube. The flight time provides additional information on the particle momentum, especially for slow particles which scatter at wider angles and thus have 


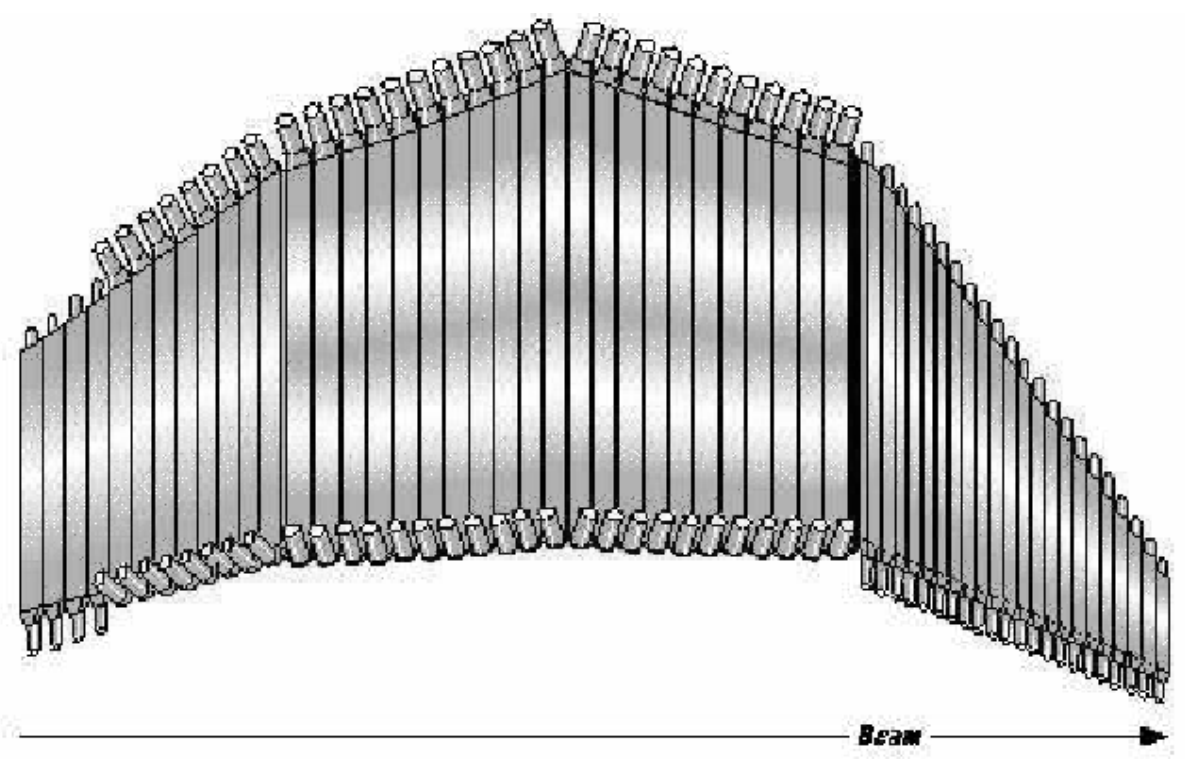

Figure 3.5: One sector of the Time of Flight Counter

lower tracking resolution.

\subsubsection{C̆erenkov Detector}

The purpose of the $\breve{C}$ erenkov Detector is to aid in the identification of electrons. A charged particle which traverses a medium at a velocity, v, which exceeds the local velocity of light in the medium, $\mathrm{c} / \mathrm{n}$, emits electromagnetic radiation. This radiation can be collected via photomultiplier tubes and constitutes the $\breve{C}$ erenkov signal that is used for online triggering and offline separation of pions and electrons. The medium inside the Cereknov can be chosen so that only charged particles with a mass as small as the electron will be energetic enough to reach the speeds needed for emission of this radiation. Perfluorbutate, $\mathrm{C}_{4} F_{10}$, is the gas used to fill the CLAS $\breve{C}$ erenkov Detector. As a result most negative pions with momentum under $2.8 \mathrm{GeV} / \mathrm{c}$ are too slow to 
emit $\breve{C}$ erenkov radiation. However, approximately $1 \%$ of all pions are detected due to primary and secondary ionization of the atomic electrons in the gas. A complete discussion of the $\breve{C}$ erenkov Detector is given in the publication The CLAS $\breve{C}$ erenkov Detector [53].

The $\breve{C}$ erenkov Detector, like all the detector packages in CLAS, is separated into 6 sectors and covers a range in $\theta$ from $0-45^{\circ}$. This is achieved by covering the front and back of the detector in mirrors and placing light collecting cones and photomultiplier tubes in the regions which lie in the shadow of the torus magnet. Each sector of the $\breve{C}$ erenkov is divided into 18 regions of $\theta$, and each $\theta$ region was again separated into two symmetric modules. This design breaks the $\phi$ region into 12 symmetric subsectors and the $\theta$ region into 18 regions, resulting in a total of 216 light modules.

Each of the 216 light modules contains two focusing mirrors, a "Winston" light collection cone, and a cylindrical mirror at the base of the cone as is shown in Fig. 3.6. The light is then detected by a 5 inch Phillips XP4500b PMT mounted at the base of the Winston cone.

\subsubsection{Electromagnetic Calorimeter}

The forward regions of the CLAS detector sectors are equipped with an electromagnetic shower calorimeter. The calorimeter was designed to perform three major particle identification tasks. The first is to help with the identification and separation of electrons and negatively charged pions. The largest source of background contamination for electron detection is the copious production of $\pi^{-}$and therefore reduction

of this systematic error is of paramount importance. The $\breve{C}$ erenkov detector assists in this separation but the efficiency drops as the momentum of the $\pi^{-}$particles becomes 


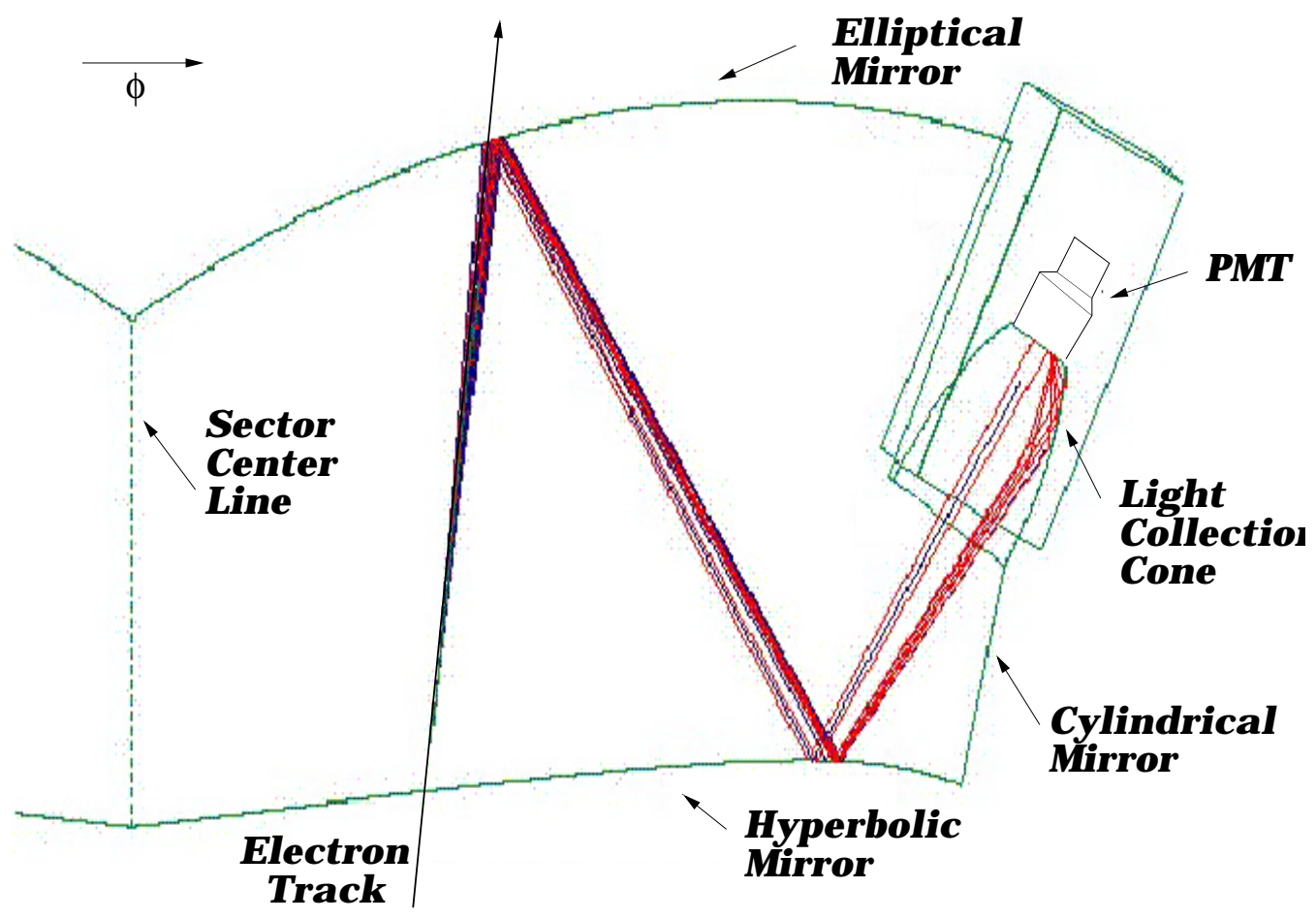

Figure 3.6: $\breve{C}$ erenkov detector mirrors, light cones, and phototubes.

large, $\sim 3.0 \mathrm{GeV}$, and the pions become energetic enough to also emit $\breve{C}$ erenkov light. The calorimeter can also be used in the reconstruction of the $\pi^{\circ}$ and the $\eta$ by detection and identification of their $2 \gamma$ decays. Finally the EC was designed to aid in the detection of neutrons and subsequently the separation of the neutron and photon. A complete description of the construction and performance of the calorimeter is given in The CLAS forward electromagnetic calorimeter paper[54].

The physics behind a calorimeter lies in the way particles of different mass and charge interact with the material inside the detector. The CLAS EC is made of lead and scintillator. Scattered particles interact with the nucleus and atomic electrons in lead and deposit energy in the form of light which is collected by the scintillator. 
Scattered particles deposit energy in the EC primarily via two process, ionization and radiation. All charged particles will interact with the atomic electrons, causing emission of photons either through excitation or ionization. Electrons with energies of a few GeV lose $99 \%$ of their energy through radiation. The electron is deflected by the Coulomb field of the nucleus (and the atomic electrons screening it) and emits bremsstrahlung radiation. These photons decay to electron-positron pairs, which in turn radiate photons. The cycle continues until the $\mathrm{e}^{+} e^{-}$pairs are not energetic enough to continue the shower. Particles which are more massive than the electron have an extremely small bremsstrahlung cross-section at these energies and energy loss from radiation is considered negligible. Ionization and radiation produce distinctly different signals in the EC. Electrons deposit a constant fraction of their total energy largely in the front half of the EC. The energy deposited by heavier particles is more dependent on the amount of lead and is more consistently deposited throughout the EC.

The forward calorimeter is constructed from alternating layers of $10 \mathrm{~mm}$ thick BC412 scintillator strips, and 2.2mm lead sheets. In order to minimize shower leakage each successive layer increases in area, giving each sector of the EC a truncated pyramid shape with a projected vertex at the CLAS target point 5 meters away. This construction results in approximately 0.27 of the energy of the scattered electron to be deposited in the scintillator.

The calorimeter is composed of 13 submodules, each consisting of a U, V and W lead/scintillator layer. The U,V and W layers are oriented at $120^{\circ}$ to each other, providing stereo information on the location of energy deposition (see Fig. 3.7). The 13 submodules are divided into an inner and and outer stack which contain 5 and 8 
submodules respectively. Each scintillator layer is composed of 36 strips. The number of strips in each layer (36), the number of stacks (2) and the number of $\mathrm{U}, \mathrm{V}$ and $\mathrm{W}$ views (3) requires 216 photo multiplier tubes for each sector of the calorimeter. The PMT's are connected to the scintillators via a fiber optic light readout system.

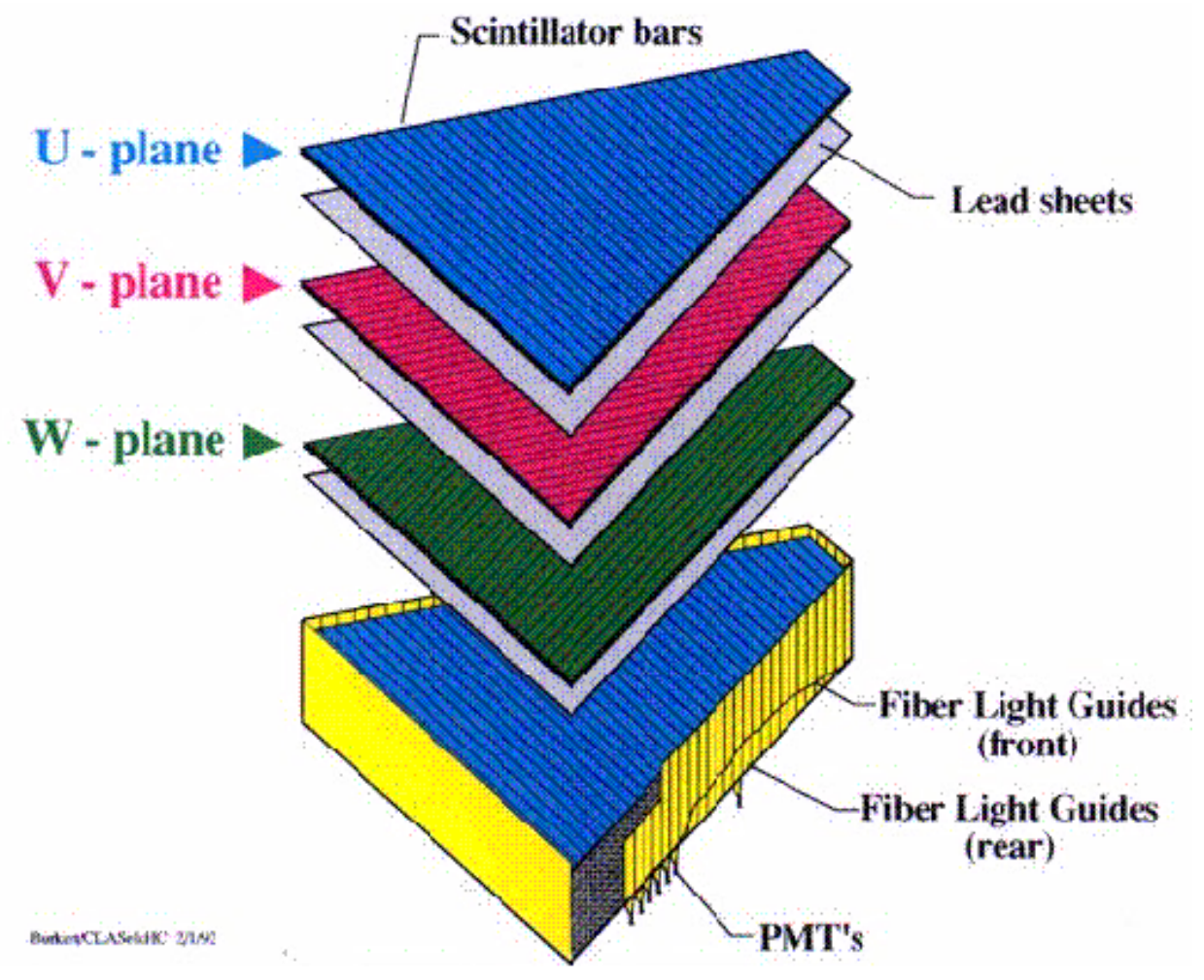

Figure 3.7: U,V and $\mathrm{W}$ layers of the Electrocalorimeter

The forward Electromagnetic Calorimeters cover the polar angle, $\theta$, out to $45^{\circ}$. The Large Angle Calorimeter extends this coverage to $75^{\circ}$ in two sectors of CLAS. The design goals of the LAC are much the same as for the forward EC; electron and negative pion separation, neutral particle detection and neutron and photon separation. The Large Angle Calorimeter is described fully in the publication Response to cosmic rays of the large-angle electromagnetic shower calorimeter of the CLAS 
Table 3.1: EG1 Trigger Thresholds

\begin{tabular}{|c|c|c|}
\hline Detector & Hardware $(\mathrm{MeV})$ & Threshold \\
\hline \hline EC & 150 & $600 \mathrm{MeV}$ \\
\hline CC & 38 & $\leq 1$ photo electron \\
\hline
\end{tabular}

detector [55].

\subsubsection{Data Acquisition and Trigger System}

Experimental results rest on the ability to detect the desired physical process as well as to discriminate between the reaction being studied and background reactions. The Data Acquisition System (DAQ) was designed to identify when a scattered electron has been detected by the CLAS and then record the detector responses associated with that event. It is necessary to maximize the amount of background rejected by the DAQ so that the storage space and analysis time needed to reconstruct the detected particle tracks can be minimized. This screening is implemented in the form of trigger thresholds. A trigger is designed to tell the DAQ when it should read and store the output from the detector. When a single detector package registers any type of physics event the signal is immediately sent to a pretrigger discriminator. The thresholds of these discrimminators are set according to the physics goals of the experiment. The condition for an EG1 trigger is given by the detection of an electron in the final state, which is defined as a simultaneous hit in the $\breve{C}$ erenkov and Electromagnetic Calorimeter. Table 3.1 details the hardware and physics thresholds set on the Calorimeter and $\breve{C}$ erenkov detector during the EG1 run. 


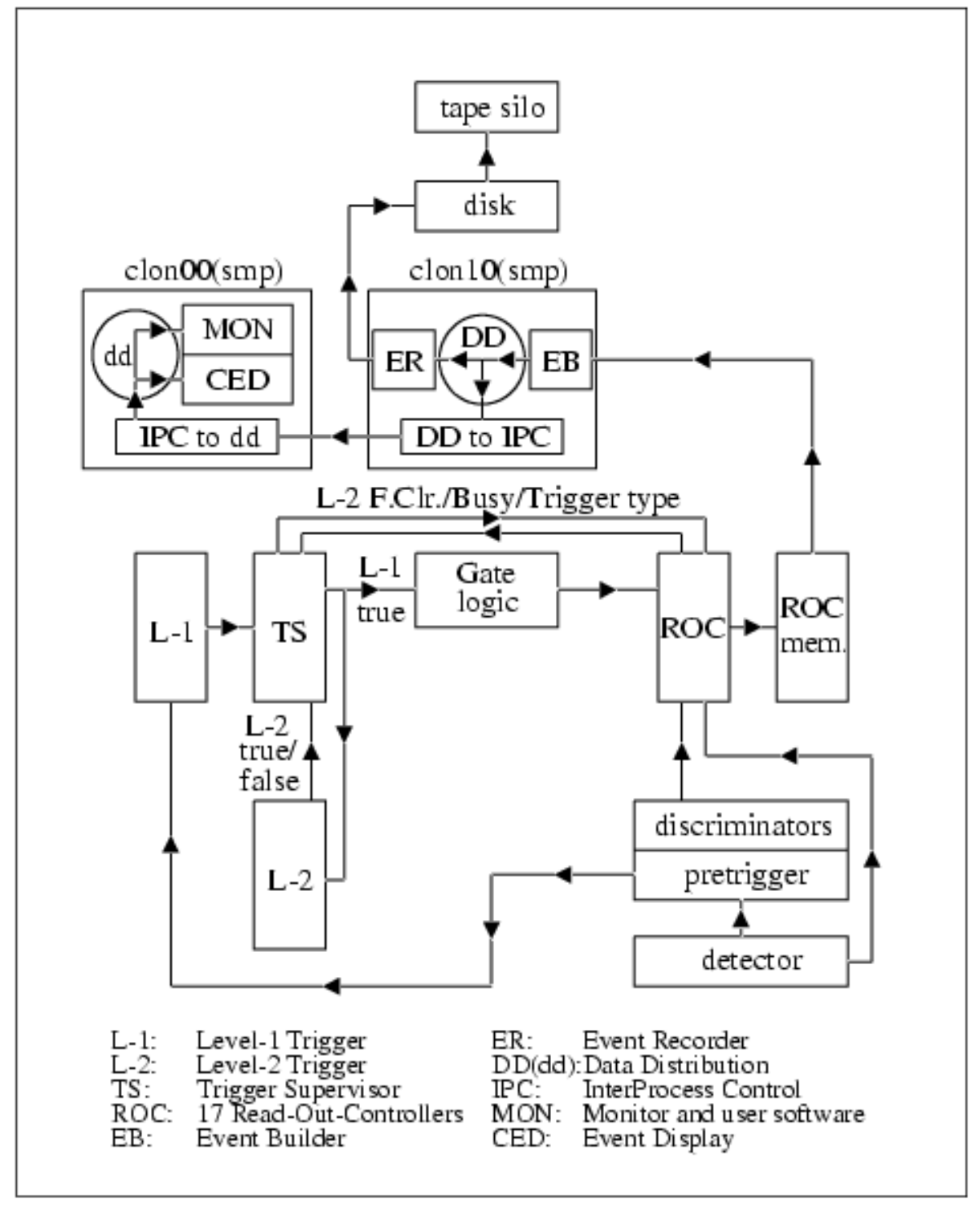

Figure 3.8: The DAQ and trigger system for EG1 
The selection of the trigger threshold also defines the resulting $\mathrm{W}$ and $Q^{2}$ range of the electrons that enter the data set. Low momentum particles contribute more heavily to the higher W spectrum and therefore reducing the trigger threshold of the EC allows a wider $\mathrm{W}$ and $Q^{2}$ range to be observed. This choice must be weighed against the reduction of $\pi^{-} / e^{-}$discrimination. Depending on the beam and torus setting, a good electron in the final state occurred in 40-60\% of the triggers taken. The remainder of the events were caused by noise or particles that hit the support structure of the EC or CC and caused a shower. These events are rejected during the reconstruction process because their track do not lead back to the target.

If a signal satisfies the pretrigger discriminator it is passed onto the Trigger Supervisor. At this stage there are two Trigger Levels. Level I accepts and rejects signals based on the response from detectors with fast readout times, such as the $\breve{C}$ erenkov, EC and the TOF. Level II trigger is based on slower detectors like the drift chamber. For EG1 the level I \& II triggers accepted events if they could be identified with at least one track in the drift chambers. Once accepted the Trigger Supervisor passes the event along to the Event Builder and the Event Recorder by communicating with the Read Out Controllers (ROCs) via 17 FASTBUS crates. In this process the signals from each detector package are converted from analog to digital and identified as an event. Events are recorded on local disk and later transfered to the tape silo at Jefferson Lab. A schematic of this process is shown in Fig. 3.8.

The limited size of the DAQ buffer and the rate at which the buffer transfers data to the local disk reduces the amount of time the DAQ can receive new data. The percentage of time that the DAQ is actually open is called the "live-time". During the EG1 run the beam intensity was chosen to maintain a live-time of $\sim 85-90 \%$ 
with a data taking rate of $1500-2200 \mathrm{kHz}$. In order to ensure that the detector is performing optimally there is an Online Monitoring System which allows shift takers to view detector and DAQ responses as data taking is occurring. Events from the main Data Distribution ring are copied over to a satellite computer and then displayed on the computer clon00 via the CLAS Event Display (CED) and monitoring (MON) GUI's.

\subsection{Polarized Target}

The polarized protons or deuterons for the EG1 run were supplied by a dynamically polarized solid state target, see Fig. 3.9. The target was required to fit inside CLAS, while ensuring that the region one drift chambers would not be damaged from cryogens or any other by-product of a cryogenic target (such as ice or water). The resulting target is composed of a $5 \mathrm{~T}$ superconducting magnet in a Helmholtz configuration which allows for a wide forward scattering angle as well as $90^{\circ}$ scattering, and a Helium-4 evaporation refrigerator which was located outside of the drift chambers and entered the target chamber at an $30^{\circ}$ angle with the beamline. The target material was polarized using microwaves at a frequency of $140 \mathrm{GHz}$. The polarization was monitored using a Nuclear Magnetic Resonance System (NMR). The theory behind polarized targets as well as a detailed description of the subsystems used during the EG1 run will be discussed in the following sections. 


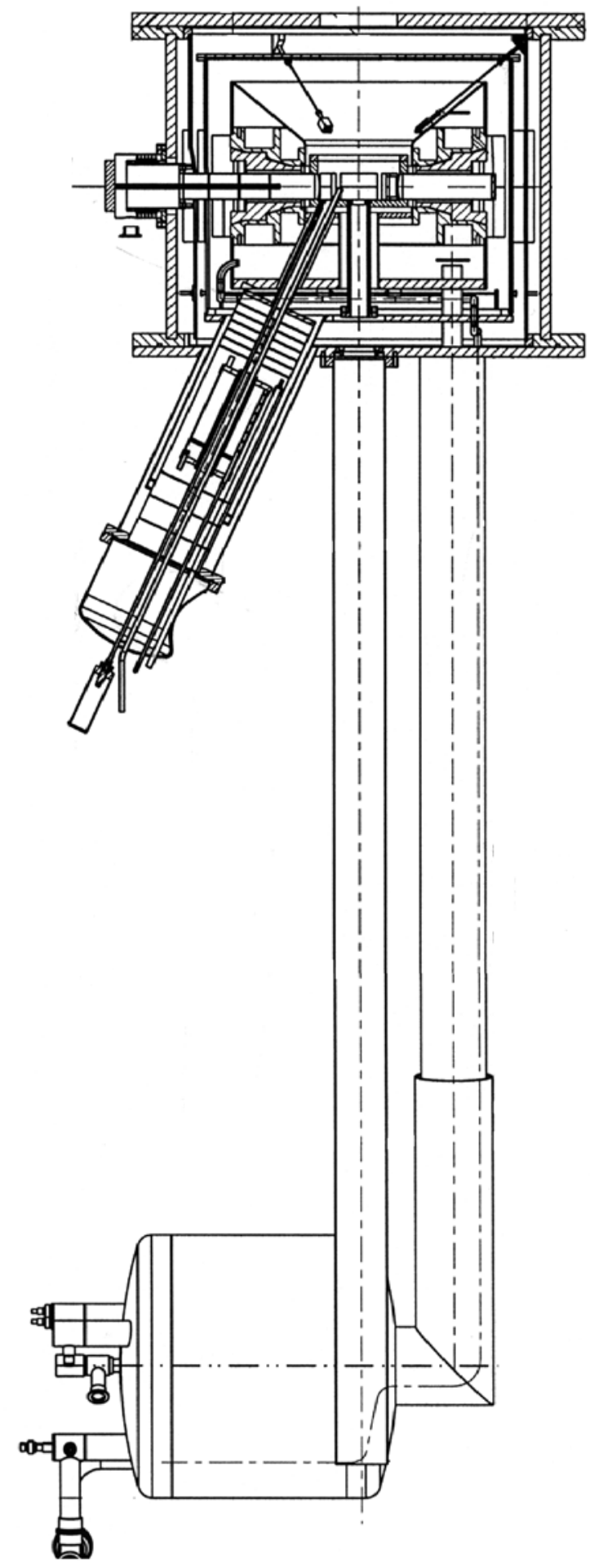

Figure 3.9: Side view of target, refrigerator and service module which feeds helium to the magnet and separator. 


\subsubsection{Theory of Solid Polarized Targets}

The spins of an ensemble of free nucleons, physically constrained by a solid lattice, will have no preferred direction within the lattice. If the solid is placed in an external magnetic field, at low temperatures, then the magnetic moments, $\mu_{N}$, of the nucleons interact with the field and the spins will tend to align themselves with the field. The percentage of total spins aligned in the direction of the field depends on the strength of the magnetic field, B, and the amount of thermal energy in the lattice, which in turn is determined by the lattice temperature, $\mathrm{T}_{\text {lattice. }}$. In order to determine the polarization of the system it is necessary to define the partition function Z:

$$
Z=\sum_{j_{i}=-J}^{J} e^{-\frac{j_{i} H}{k T_{s}}}
$$

The Hamiltonian of the system is $H=-\overrightarrow{\mu_{N}} \cdot \vec{B}, \mathrm{k}$ is Boltzmann's Constant, $\mathrm{j}_{i}$ is all possible projections of the nucleon spin on the axis of the B-field and $T_{s}$ is the spin temperature of the system[57]. The polarization is found by differentiating the partition function:

$$
P=\frac{\partial Z}{\partial\left(H / k T_{s}\right)}
$$

In the case of a spin $1 / 2$ system (proton) and a spin 1 system (deuteron), the polarization is defined as:

$$
P_{1 / 2}=\tanh \frac{\mu B}{k T_{s}} \quad P_{1}=\frac{4 \tanh \frac{\mu B}{2 k T_{s}}}{3+\tanh ^{2} \frac{\mu B}{2 k T_{s}}}
$$

When the ensemble of nucleons is in a state of thermal equilibrium with the lattice, the spin and lattice temperatures are equal. Inserting a target temperature, $T_{s}=1.2$ $\mathrm{K}$ and a B-field of $5 \mathrm{~T}$ into eqn. 3.3 results in a polarization of $0.42 \%$ for the proton. 
Clearly this is an unacceptably low polarization for any scattering experiment. The small polarization is due only to the size of the magnetic moment of the proton. In comparison, electrons have a polarization of $99 \%$ at the same field and temperature. It is the high polarization of electrons under these conditions that eventually allows the nucleons in the target also to be polarized to a much higher degree. These techniques and the physics underlying them will be discussed in the next section.

\subsubsection{Dynamic Nuclear Polarization}

Dynamic Nuclear Polarization (DNP) is used to induce higher nuclear polarizations in solid state targets. This is accomplished by first introducing free electrons to the ensemble and then transferring the high electron polarization to the nucleons. Consider a system of free electrons and protons placed in a magnetic field. The Hamiltonian now becomes:

$$
H=-\overrightarrow{\mu_{e}} \cdot \vec{B}-\overrightarrow{\mu_{P}} \cdot \vec{B}+H_{i n t}
$$

Figure 3.10 demonstrates the splitting of the electron and proton energy levels in the magnetic field. In a system where the protons and electrons do not interact the only allowed transitions are those of energy $\triangle E=2 \mu B$, which corresponds to a frequency of $\nu_{p}=213 \mathrm{MHz}$ for the proton and $\nu_{e}=140 \mathrm{GHz}$ for the electron when $B=5.0 \mathrm{~T}$. These energy levels allow only the flipping of a proton, states a) $\Leftrightarrow$ b)and c) $\Leftrightarrow d$ ), or an electron, states a) $\Leftrightarrow$ c) and b) $\Leftrightarrow d$ ). The two systems are not isolated, however, and therefore $H_{\text {int }}$ represents the interaction between the electron and proton spins. It is this interaction term that allows mixing of energy transitions 
for the proton and electron. This means that the proton-electron system can make the transition $\mathrm{a}) \Leftrightarrow \mathrm{d}$ ) or $\mathrm{b}) \Leftrightarrow \mathrm{c}$ ). For example if the goal is to pump protons from the $+1 / 2$ state to $-1 / 2$ state then the first step would be to irradiate the material with microwaves of frequency $140 \mathrm{GHz}+213 \mathrm{MHz}$. The electron - proton system will absorb the energy and make the transition from level a) to d), resulting in a flip of both the electron and proton spin.

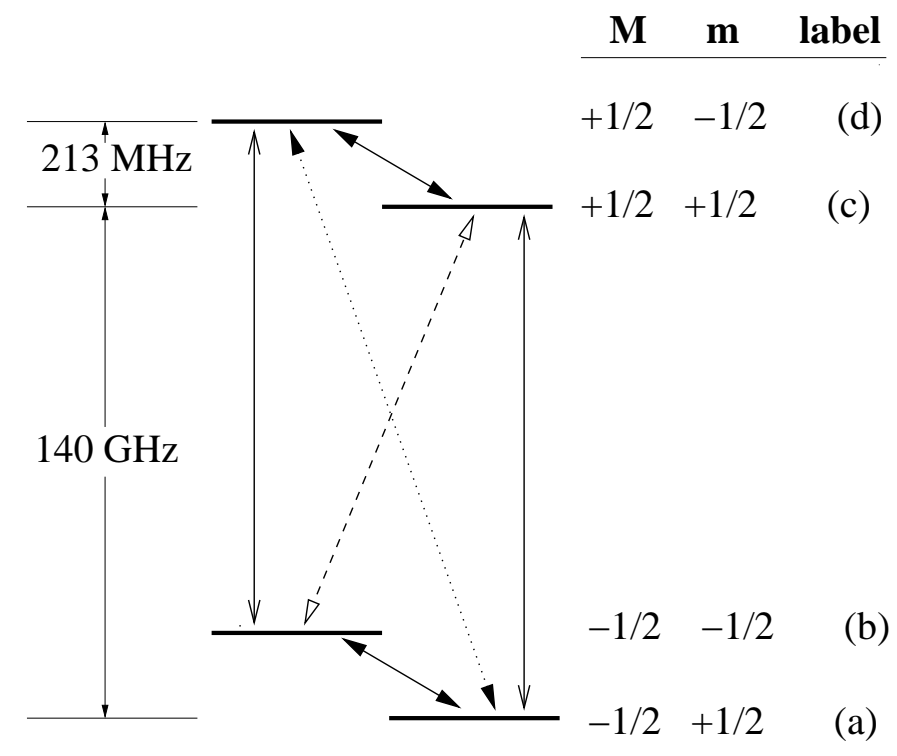

Figure 3.10: Diagram of the possible electron-proton coupled states in the "Solid State Model". $\mathrm{M}$ and $\mathrm{m}$ are the $\mathrm{z}$ components of the electron and proton spin states. 
At this point the electron, which has a much larger magnetic moment and thus stronger coupling to the lattice, will relax back to its original spin state. This relaxation time depends on the temperature of the lattice but is on the order of $10^{-3}$ seconds. The proton spin has a much longer relaxation time, ranging from several minutes to hours. The final state of the system is then b), where the proton's spin has been flipped and the electron is in it's original state and free to help pump another proton to the $-1 / 2$ state. The opposite transition is pumped in exactly the same way, the only difference is that microwaves of energy $140 \mathrm{GHz}-213 \mathrm{MHz}$ are used. This excites the proton-electron transition b) $\Leftrightarrow$ c) and then relaxation to the final state a).

A secondary process which aids in the polarization of the nuclear target is termed spin diffusion [57]. Dipole-dipole interactions between two nuclei allows the spin of one nucleon to be transfered to a neighboring nucleon. This flip-flop transfers the nuclear alignment out to regions less populated with free electrons. The nucleon whose spin was originally flipped by the DNP process, and then transfered this polarization via the flip-flop mechanism, is relaxed back to the initial state and is available to couple with free electrons again.

\subsubsection{The Equal Spin Temperature Theory}

The previous section described the DNP process in terms of the "Solid State Model". This theory defines the electron state by its interactions with only the magnetic field and nucleons in the target. In reality high concentrations of free electrons exist in the target, resulting in non-negligible dipolar interactions between electrons [58]. Although these interactions are weak compared to the Zeeman interaction they result 
in a smearing of the discrete electron states described in the solid state effect. In the Equal Spin Temperature (EST) model the electrons exist in in a band of quasicontinuous states and are described by Boltzmann Statistics where the critical temperatures are $T_{s s}$ and $T_{Z e}$, the temperature of the electron spin-spin reservoir (SSI) and electron Zeeman reservoir respectively.

In the EST model, DNP proceeds through an absorption of microwave energy by the electron SSI and Zeeman reservoir. The SSI electron reservoir can then exchange energy with the nuclear Zeeman reservoir, resulting in thermal mixing. This mixing allows a relaxation channel previously forbidden in the solid state model. This channel consists of the flip-flop of two electron spins together with a nuclear spin flip. The microwave frequency needed to induce this type of relaxation channel is very close to the Larmour frequency of the electron.

A direct consequence of the EST model is that all species of nuclei in a target should be at the same spin temperature. If this is true, it is possible to use the measured polarization of one species to extract the spin temperature and then use this temperature to calculate the polarization of any other species in the target. This method is used, for example, to estimate the ${ }^{15} \mathrm{~N}$ polarization in ammonia targets. The ability to correct for the polarizations of other target materials is necessary and allows for flexibility in the choice of target material.

\subsubsection{The Target Material}

The target material used for the EG1 run was frozen ammonia, ${ }^{15} \mathrm{NH}_{3}$ and ${ }^{15} \mathrm{ND}_{3}$. This material was chosen because of the relatively high ratio of free to bound nucleons, roughly $16.5 \%$ for ${ }^{15} \mathrm{NH}_{3}$. Ammonia has also shown potential for high polariza- 
tions, $>90 \%$ for protons and $\sim 40 \%$ for deuterons [58]. It has a good resistance to beam radiation as well, resulting in a factor of $\mathrm{e}^{-1}$ decay in polarization after $\sim 10^{16}$ electrons $/ \mathrm{cm}^{2}$ of beam. Traditionally targets composed of hydrogenated and deuterated alcohols have been used. These materials were rejected in favor of ammonia due to their smaller dilution factor and low beam resistance. However the ${ }^{15} \mathrm{~N}$ in the ammonia contains an unpaired proton which may also be polarized. Fortunately this polarization can be estimated and corrected for using the EST model discussed in the previous section.

The target material was prepared by slowly freezing the gaseous ammonia at liquid-nitrogen temperatures. The material is then crushed into granules with diameters of 1-2 $\mathrm{mm}[60]$. The motivation behind creating small beads is to reduce heating within the material. Granules increase the surface area of the material that is in contact with the liquid helium bath and are therefore cooled more efficiently than larger pieces would be. Beam heating effects must be reduced otherwise the polarization will be severely limited by the higher temperatures induced by the electron beam.

The ammonia beads were irradiated with a low energy electron beam at liquid argon temperatures in order to embed the free electrons necessary for the DNP process. The free electrons, called paramagnetic centers, are created when an electron from the beam knocks out a free proton in the material, leaving pockets of ${ }^{15} \mathrm{NH}_{2}^{-}$. The electron that was formally attached to the ejected proton is now less tightly bound and can couple to a proton in the polarization process. A minimal number of centers are needed for any DNP to occur, but an excess of centers impedes the polarization process. Therefore the irradiation dose is controlled to deliver the maximum possible polarization. Irradiation of material for the EG1 run took place at the Jefferson Lab 
FEL beamline in the summer of 1998 at liquid Argon temperatures. EG1 also used target material that had been irradiated at the SUNSHINE facility on the Stanford Campus.

Once the target material has been pre-irradiated it is ready for experimental running. EG1 is an electron scattering experiment so the material continues to be irradiated during data taking, only at colder temperatures (4K compared to $40 \mathrm{~K}$ ). This runtime irradiation produces more paramagnetic centers and eventually a threshold is reached and the polarization starts to decrease. At this time it is necessary to remove the paramagnetic centers by a process termed "annealing". Annealing the target material requires the proton material to be raised to temperatures of $\sim 85 \mathrm{~K}$ and the deuteron material to $\sim 95 \mathrm{~K}$. The excess free radicals in the ammonia are recombined and the number of paramagnetic centers is once again reduced. The possibility of annealing also makes ammonia a more desirable target than the traditional alcohols which cannot be restored in this manner. Hall-B's lower currents reduced the need to anneal the target. Due to the accelerator schedule the target was never annealed, the material was simply replaced when needed during a convenient down time.

\subsubsection{The Target Magnet and Cryostat}

The EG1 target cryostat was designed to house and cool a $5 \mathrm{~T}$ superconducting magnet as well as the experiment target material. The magnet is a Helmholtz pair, built by Oxford Instruments, and constructed of Niobium-Titanium alloy. The magnet was designed to provide a field, uniform to $10^{-4} \mathrm{~T}$, located in the geometric center between the two coils. This uniform field region defines the geometric limits of the target cell and must be directed longitudinally, that is along the beamline. The direc- 


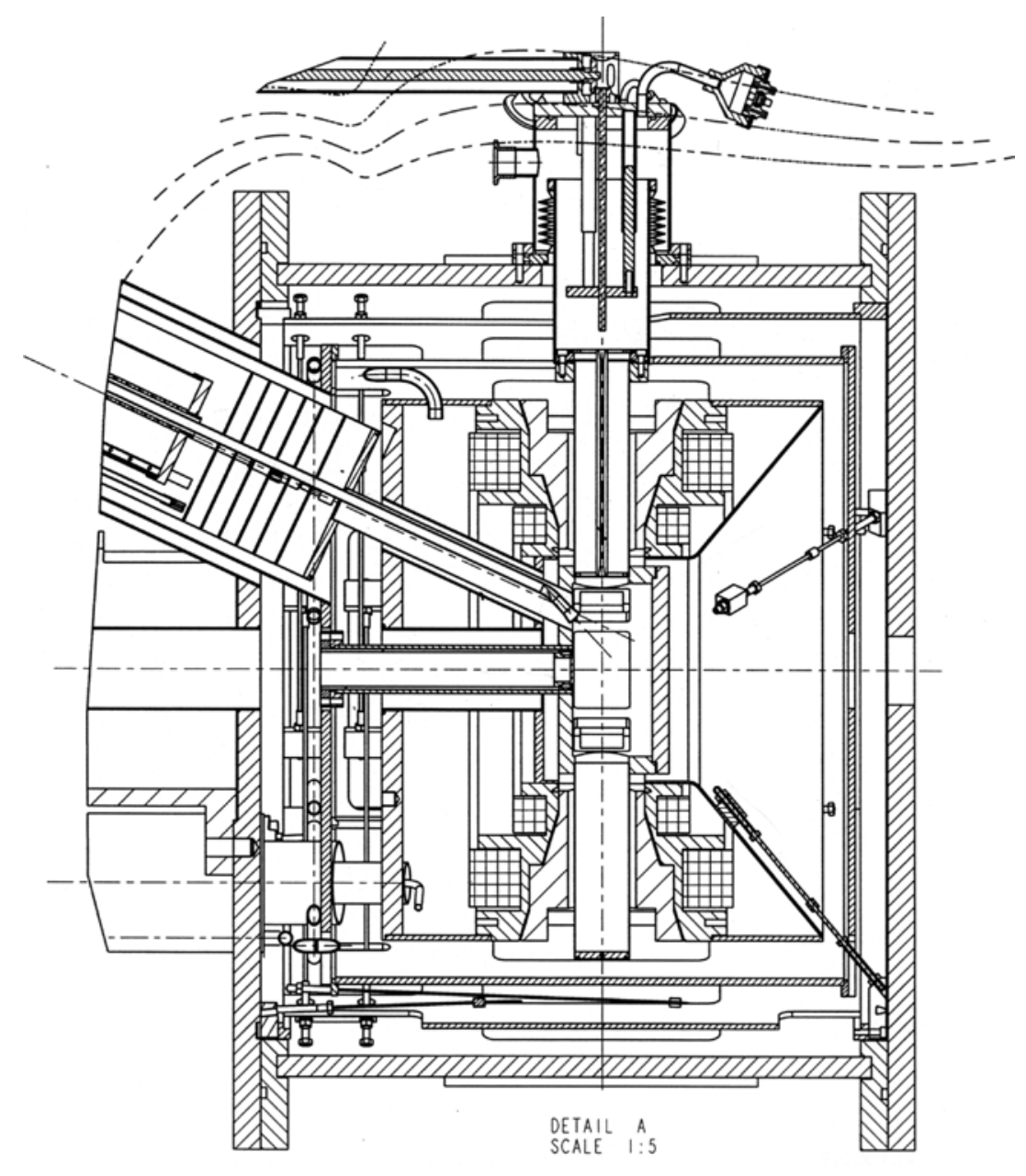

Figure 3.11: Side view of the target magnet and cryostat. $90^{\circ}$ scattering occurs into and out of the page. The banjo is situated in the center where the end of the refrigerator enters at a $30^{\circ}$ angle with the beamline. 
tion of the field determines the direction of the polarization of the nucleons and since the beam is longitudinally polarized it is necessary that the nucleons are as well. The magnet is housed inside a stainless steel can which holds the $4 \mathrm{~K}$ liquid helium used to cool the coils to superconducting temperatures. The coil and can are designed to maximize the use of the CLAS detector by allowing for $\pm 50^{\circ}$ scattering in $\theta$ as well as $90^{\circ} \pm 17^{\circ}$ scattering which occurs between the two Helmholtz coils.

The service module is a pod that contains a reservoir of helium which is used to feed the target coils as well as provide a means of exhaust for the magnet boil off. The pod is designed to sit outside of CLAS in order to limit the exposure of the detector to cryogens. It is connected to the magnet can and cryostat by a long pipe, through which all cryogens are delivered and expelled. Two ports located at the top of the pod house the magnet leads. These leads are hermetically sealed and allow for attachment of power supply leads which are used to control the current in the coils. The power supply also controls the superconducting switch. When it is necessary to ramp the magnet or change the current in the coils the superconducting switch electrically connects the magnet coils to the power supply leads. When the magnet is in persistent mode the switch is closed. This allows the magnet to operate as a closed system, meaning the coils no longer see the leads, part of which are at "normal" temperatures. This reduces the amount of helium gas boil-off in the cryostat. In persistent mode, the current slowly decays at a rate which depends on the amount of impurity introduced into the superconducting coil joints during construction. The EG1 target had a decay rate of 1 part in $10^{6}$ per hour.

There are three additional ports on the service module which are used for helium supply and exhaust. One port is connected to an external dewar of liquid helium and 
it feeds, via a tube, directly to the magnet can. A valve, also located on the pod controls the path of the helium exhaust. When the valve is closed the helium boiloff vapor simply exits out of the bottom of the magnet can and up through the pod. When the valve is open the vapor is forced to first travel through a copper tube which is coiled on the back of a the stainless steel radiation shield surrounding the magnet can. This alternate path provides a higher resistance and allows the extremely cold gas to cool the radiation shield, thus providing a more effective heat shield for the magnet. During the run the valve is half open in order to maximize the radiation shield cooling while minimizing the pressure in the can due to the boil-off.

The target chamber, called the "banjo", is situated in the center of the magnet coils, although outside and thermally disconnected from the magnet can. The banjo is composed of a round cylindrical chamber, $200 \mathrm{~mm}$ in diameter, and attached at the top to a stainless steel pipe which extends to the top of the cryostat (see Fig 3.11). The pipe provides a means to insert the target stick, which holds the target material, into the center of the banjo and hence into the uniform magnetic field. The target chamber, also constructed of stainless steel, was designed to have cylindrical symmetry in order to provide optimal $90^{\circ}$ scattering conditions. The banjo also provides an entry port for $1 \mathrm{~K}$ liquid helium from the refrigerator, which will be discussed in the following section. The banjo has an entrance and exit window as well as six radial windows which are geometrically aligned with the six sectors of the CLAS. The exit window is concave, creating an inverted cone with a flattened circle for the center. The concave exit window design minimized the amount of helium the forward scattering particles were required to travel through. Three radiation shields were installed outside the banjo in order to reduce the heat load on the target and 


\begin{tabular}{|l|c|c|c|}
\hline Description & Beamline location & material & thickness \\
\hline \hline cryostat entrance window & -120.5 & $\mathrm{Al}$ & 71 microns \\
\hline cryostat heat shield & -81.5 & $\mathrm{Al}$ & 10 microns \\
\hline banjo entrance window & -55.75 & $\mathrm{Al}$ & 71 microns \\
\hline $\begin{array}{l}\text { upstream space between } \\
\text { banjo window and target } \\
\text { entrance window }\end{array}$ & -55.625 & $\mathrm{LHe}$ & $0.25 \mathrm{~cm}$ \\
\hline target entrance window & & $\mathrm{Al}$ & 25 microns \\
\hline target material & -55.5 & $\mathrm{NH}_{3}, \mathrm{ND}_{3}, \mathrm{C}_{12}, \mathrm{LHe}$ & 1 cm \\
\hline target exit window & -55.0 & $\mathrm{kapton}$ & 50 microns \\
\hline $\begin{array}{l}\text { downstream space between } \\
\text { target exit window and } \\
\text { banjo exit window }\end{array}$ & -54.5 & $\mathrm{Al}$ & $0.25 \mathrm{~cm}$ \\
\hline banjo exit window & -54.375 & $\mathrm{Al}$ & 71 microns \\
\hline 4K shield & -54.25 & $\mathrm{Al}$ & 20 microns \\
\hline radiation shield & -46.0 & $\mathrm{Al}$ & 20 microns \\
\hline space blanket & -36.0 & $\mathrm{Al}-\mathrm{Mylar}-$ Cerex & 12 sheets \\
\hline OVC exit window & -35.0 & $\mathrm{Al}$ & 71 microns \\
\hline
\end{tabular}

Table 3.2: Material in the Beamline

magnet can. The entire target assembly (banjo, magnet can and heat shields) is surrounded by the outer vacuum can (OVC). The OVC provides a frame for the large forward scattering window and six rectangular radial scattering windows, as well as ensuring a vacuum of $10^{-} 7$ Torr on the inside. Table 3.2 contains a list of all the materials, their thickness, and positions along the beamline, that the beam passes through when entering and exiting the target cryostat.

The polarized target magnet replaced the mini-torus which is used in most HallB experiments. The polarized target affects the path of the scattered particles by rotating their phi projection, while leaving their polar angle virtually untouched. The target field falls off rapidly but still extends into region 1 drift chambers. Because 
of these changes in magnetic field it was necessary to include a polarized target field map in the reconstruction programs.

\subsubsection{The Helium-4 Evaporation Refrigerator}

The EG1 target used a helium-4 evaporation refrigerator, in tandem with a three stage pump system, in order to provide a $1 \mathrm{~K}$ environment for the target material. The space restrictions defined by the Region I drift chambers of the CLAS detector required that the refrigerator enter into the banjo at a $30^{\circ}$ angle (see Fig. 3.9). This had the advantage of placing most of the electronics and controls for the refrigerator outside of CLAS. The refrigerator slides into a stainless steel port that leads to the banjo on the downstream side. The back of the refrigerator is connected to a large diameter stainless steel tube which leads to six pumps. The first stage is composed of two mechanical pumps, Alcatel 2063H, with pumping speed of $63 \mathrm{~m}^{3} / \mathrm{h}$. The second and third stages consist of two Alcatel Ruvacs RSV600 and RSV2000 pumps, with pumping speeds of 600 and $2000 \mathrm{~m}^{3} / \mathrm{h}$ respectively.

The main components of the refrigerator are the separator pot, two sets of heat exchangers, and two valves which deliver the cooled helium to the target (see Fig 3.12). The refrigerator receives $4.2 \mathrm{~K}$ helium from a vapor cooled transfer line from the service module. The liquid is pumped into the separator via a compressor pump, called the separator pump, which is attached to the vapor exit port of the pot. The separator pot is a copper cylinder which is divided into an upper and a lower chamber by a plate of copper sinter. The $4.2 \mathrm{~K}$ liquid helium enters the upper chamber of the separator and is immediately cooled by the sub-atmospheric pressure (600 mbar). The liquid condenses through the sinter and collects in the bottom chamber while the gas 


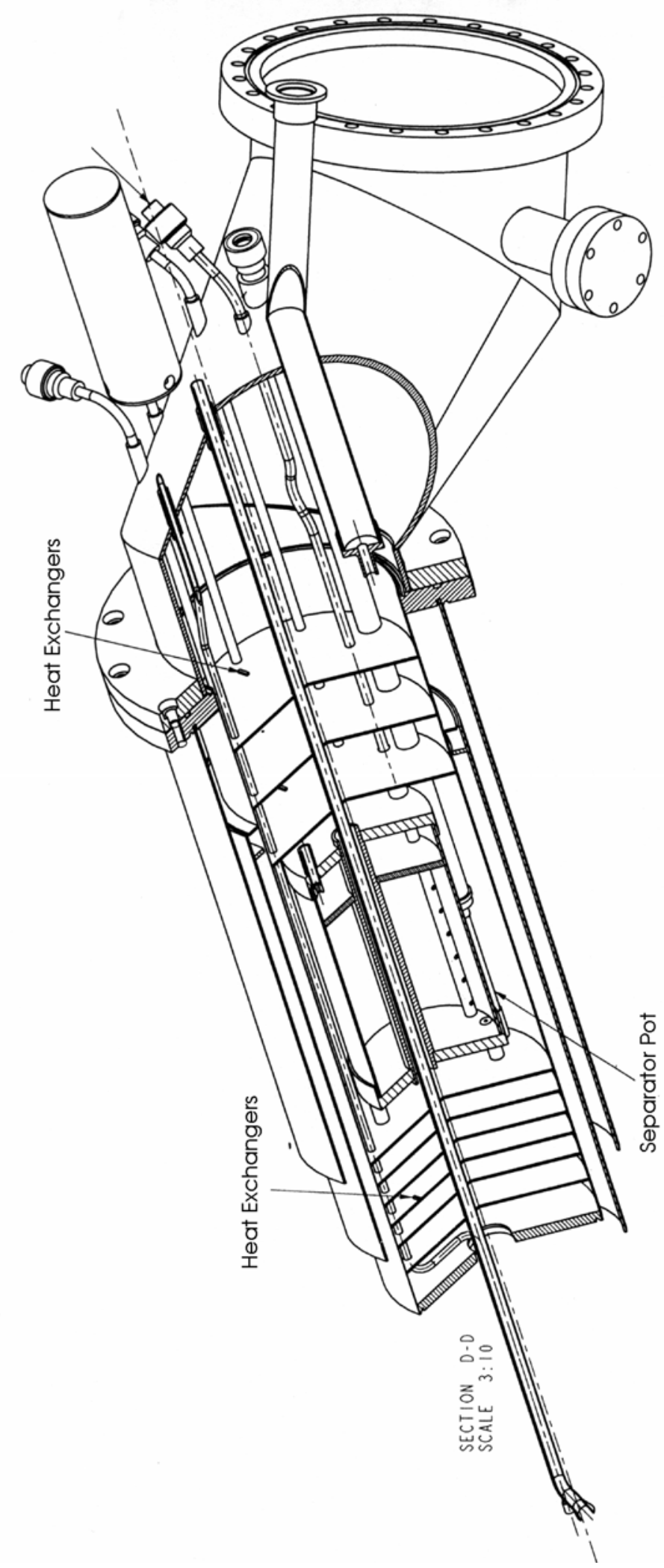

Figure 3.12: The refrigerator removed from the target cryostat. 
is pumped out of the top chamber by the separator pump. The exiting cold vapor travels out through copper tubes which are thermally connected to a set of radiation baffles. These baffles are cooled by the outgoing vapor, providing a radiation shield for the separator.

Liquid that has accumulated in the separator has two means of reaching the target. The first is through the bypass valve. This valve controls the flow of liquid helium from the separator into a stainless steel tube which extends directly into the banjo. The bypass valve is only used during initial cool down periods because there is no additional cooling of the helium between the separator and the banjo, resulting in liquid ranging in temperature from $2-4 \mathrm{~K}$. The second means of delivering helium to the target is through the run valve. The run valve releases liquid helium from the pot into a long copper tube which is coiled around the lower set of heat exchangers. The liquid is delivered to the banjo, boils off and is pumped out by the three stage pump system through the heat exchanges located below the separator. The thermal connection between the copper tubes and the heat exchangers, which are cooled by the exiting helium vapor, result in liquid helium of temperatures $<4 \mathrm{~K}$ to be delivered to the banjo. In the banjo, the reduced pressure created by the pumps, reduces the helium temperature to $1 \mathrm{~K}$. Eventually when the system is completely cooled down and in normal operating mode the run valve is kept at a constant value which maintains a steady level of helium in the banjo.

The refrigerator is equipped with various sensors to monitor the temperature and flow of cryogens through the separator and heat exchangers. The refrigerator also houses the He-3 vapor pressure bulb which is used to determine the temperature of the liquid helium bath in the banjo. The vapor pressure of He-3 is the most accurate 
method of determining the temperature in the banjo and thus in the targets.

\subsubsection{The Target Insert}

The target insert was designed and fabricated by the Genova Group (INFN) and is shown in Figure 3.13. The target insert has a very simple purpose: to hold the $\mathrm{NH}_{3}$ and $\mathrm{ND}_{3}$ target cells in the center of the banjo entrance window. The design and construction of such an instrument however is no trivial matter. The target stick was inserted into the banjo from the top of the Outer Vacuum Chamber. This allowed the ladder structure of the insert to be aligned perpendicular to the incoming beam. This configuration requires that the top of the insert reside at room temperature and the bottom, only half a meter away, be immersed in a $1 \mathrm{~K}$ liquid helium bath. The insert is a potential heat leak and had to be engineered to maximize strength, for repeated insertion and linear motion, while minimizing the amount of high conductivity material in the structure. Additionally the insert houses temperature instrumentation for the targets as well as the NMR system used to monitor the polarization.

The target insert consists of two aluminum rails which hold four target cells. During the EG1 run the two upper cells could have been filled with $\mathrm{NH}_{3}$ or $\mathrm{ND}_{3}$ since a proton and deuteron coil were attached to both cells. The third cell was a carbon target and the fourth an empty target. Each target cell was constructed of PCTFE (kel-F), a plastic material with a moderately high resistance to radiation and a lack of polarizable nucleons which could contaminate the proton NMR signal. All the targets are $1.5 \mathrm{~cm}$ in diameter and $1.0 \mathrm{~cm}$ in length and $0.2 \mathrm{~mm}$ wall thickness, except for the carbon target which was $2.2 \mathrm{~mm}$ instead of $1.0 \mathrm{~cm}$ in length. This length was chosen to match approximately the radiation length of the ammonia targets. The entrance 


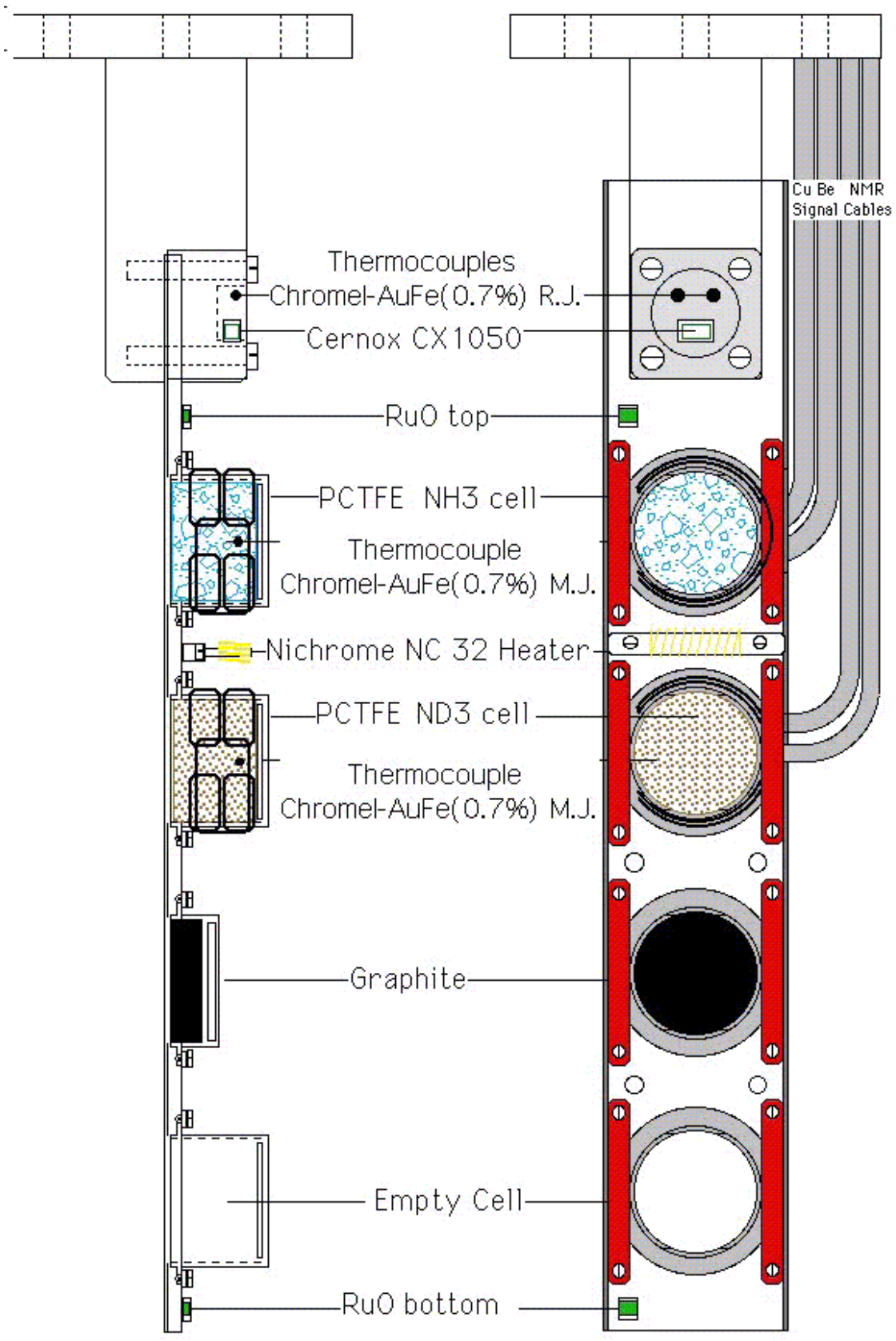

Figure 3.13: Front and side view of the target insert 
to all the target cells was covered by a $25 \mu \mathrm{m}$ aluminum foil. The exit windows were constructed of $50 \mu \mathrm{m}$ kapton foil which was put in place after filling the cells with ammonia. Kapton was chosen for the exit window due to it's strong resistance to radiation damage.

The aluminum railings attach near the top to a cylindrical brass plate. The brass disk was thermally connected via a copper spring to the $75 \mathrm{~K}$ radiation shield surrounding the magnet. This heat sink provided a thermal break in the $273 \rightarrow 1 \mathrm{~K}$ temperature gradient along the stick and thus helped reduce the heat load on the banjo. The brass disk was attached to a cylindrical stainless steel plate, which sat on top of the OVC, by a stainless steel threaded rod. This rod was attached to a stepper motor which allowed the insert to be raised and lowered so that the desired target cell could be positioned in the beam.

Coaxial cables for the NMR and temperature instrumentation entered through the top plate as well. Each proton and deuteron target cell was equipped with one junction of a Gold/Chromel thermocouple, used to monitor the temperature during anneals. The two reference junctions were placed above the targets cells and next to a cernox resistor which provided the reference temperature. A helium level probe was attached to the top of the stainless steel insert plate and extended down into the bottom of the banjo, parallel to the insert ladder. Two $1 \mathrm{~K} \mathrm{RuO}$ chip resistors were placed directly above the top target and below the bottom target. These resistors provided information about the level of the liquid helium in the banjo. A heater was situated between the two ammonia targets in order to allow for annealing of either target. The design of NMR coils and cables will be discussed in the NMR section. 


\subsubsection{The Microwave System}

Microwave irradiation of the target material is necessary for the DNP process. The microwaves are produced by a Varian Extended Interaction Oscillation Tube (EIOVKT2438P5). The EIO tube consists of a filament which emits electrons that are then accelerated through a resonant cavity. The design of this cavity only permits emission of radiation of a specific wavelength. This characteristic wavelength can be altered slightly, $\pm 2 \%$, by changing the length of the resonant cavity. For the EG1 run frequency of the microwaves was approximately $140 \mathrm{GHz}$.

The EIO tube output is guided along an air-filled $140 \mathrm{GHz}$ rectangular waveguide which makes a transition and attaches to a cylindrical waveguide located at the top of the refrigerator. The cylindrical waveguide, partially filled with liquid helium, extends down into the banjo and flares out into a horn which is designed to spread the microwaves uniformly over the target material. In order to seal the inside of the cylindrical waveguide off from the outside atmosphere, and limit possible air and water leaks into the refrigerator, a piece of FEP (a Teflon film) was inserted between the cylindrical and rectangular waveguide pieces. A tiny hole was drilled in the waveguide near the entrance to the refrigerator, ensuring that helium which entered the horn in the banjo would be pumped out before being evaporated near the upper (and warmer) and of the refrigerator. Unfortunately this tiny hole possibly allowed water, which leaked in through the waveguide seal at the top of the refrigerator, to enter the waveguide, freeze and eventually reduce the effective power of the microwaves at the banjo.

Immediately downstream from the EIO output a $30 \mathrm{~dB}$ coupler was inserted so that part of the microwaves could be siphoned off and directed to a frequency meter 
and power meter. Each EIO tube has a characteristic frequency vs power curve, meaning some frequencies in the tube's range have more power output than others. In general the tube was capable of a $20 \mathrm{~W}$ power output at the optimal frequency. The power is reduced by the length and shape of the waveguide, which attenuates 4 $\mathrm{dB}$ of power per foot. At the beginning of the EG1 run $0.9 \mathrm{~W}$ of power was delivered to the banjo. Due to the accumulation of air or water ice in the cylindrical waveguide over time, this output was reduced to $0.1-0.2 \mathrm{~W}$ by the end of the run. The only way to remove this ice is to completely warm up the refrigerator - a process which was not feasible during the EG1 run.

\subsubsection{The Principles Behind Nuclear Magnetic Resonance}

A Nuclear Magnetic Resonance System (NMR) was used to monitor the polarization of the target material. The NMR polarization results were not ultimately used in the EG1 analysis, instead the beam and target polarizations were extracted from the elastic peak. However it was necessary to monitor the polarization so that beam time was used only on highly polarized targets. It also served as a qualitative check on the elastic asymmetry results.

As discussed in the preceding sections, the spins of the free nucleons in the target material are pumped preferentially in a direction either aligned or anti-aligned with the B-field $\left(B_{0}\right)$. The spins of the nucleons precess about the direction of $B_{0}$ with a frequency $w_{0}=\frac{e B_{0}}{2 m c}$, also known as the Larmour frequency[64]. The NMR system introduces a small oscillating magnetic field, $B_{1} \cos w t$, in the plane perpendicular to $B_{0}$. This perturbation induces a precession of the nuclear spins at a frequency $w_{1}=\frac{e B_{1}}{2 m c}$ around the direction of the new field $B_{1}$. The direction of the spins in the 
target are now dependent on $w_{0}, w_{1}$ and the frequency $\omega$ at which $B_{1}$ oscillates[64]. $B_{1}$ is orders of magnitude smaller than $B_{0}$ however and doesn't create a noticeable disturbance in the polarization of the nuclear spins unless $\omega=2 \omega_{0}$. At this resonance frequency the system of nuclear spins absorb the energy introduced by the the oscillating field and as a result flip their spin direction. The flipping of nuclear spins naturally affects the magnetic susceptibility of the sample. In an oscillating field the susceptibility is composed of a real (dispersive) and imaginary (absorptive) component.

$$
\chi(\omega)=\chi^{\prime}(\omega)+i \chi^{\prime \prime}(\omega)
$$

The polarization of the sample is directly proportional to integral of the absorptive susceptibility over all frequencies[59].

$$
P=\frac{2}{\mu_{0} \pi \hbar \gamma^{2} N J} \int_{0}^{\infty} \chi^{\prime \prime}(\omega) d \omega
$$

where $\gamma$ is the nuclear gyromagnetic ratio, $\mathrm{J}$ is the spin of the species being measured, and $\mathrm{N}$ is the spin density of the material. The primary goal of the NMR system is to induce a small fraction of the nuclear spins to flip in order that the change in the absorptive part of the magnetic susceptibility may be measured and the polarization of the sample deduced.

The oscillating magnetic field, $B_{1}$, was introduced to the target by placing a small coil around the outside of the ammonia cell. The coil was designed to provide a magnetic field in the $\pm \mathrm{x}$ direction in the Hall-B coordinate system. The coil also functions as the inductor in an alternating current RLC circuit. The inductance depends not only on the design of the coil but also on the susceptibility of the material 
around the coils.

$$
L(\omega)=L_{0}[1+4 \pi \eta \chi(\omega)]
$$

where $L_{0}$ defines the inductance of the coil when the material is completely unpolarized. The filling factor, $\eta$, is a parameter ranging from 0 to 1 , which describes the coupling of the material to the coil. The total impedance of the coil is defined as:

$$
\begin{aligned}
Z_{\text {coil }} & =R_{\text {coil }}+i \omega L(\omega) \\
& =R_{\text {coil }}+i \omega L_{0}\left(1+4 \pi \eta\left(\chi^{\prime}(\omega)+i \chi^{\prime \prime}(\omega)\right)\right] \\
& =R_{\text {coil }}-4 \pi \omega L_{0} \eta \chi^{\prime \prime}(\omega)+i\left[\omega L_{0}\left(1+4 \pi \eta \chi^{\prime}(\omega)\right)\right]
\end{aligned}
$$

$Z_{\text {coil }}$ is the physical quantity which needs to be measured in order to extract the absorptive inductance. The electronics needed to do this will be described in the following section.

\subsubsection{Experimental Setup of the NMR System}

The heart of the NMR system is the continuous wave Liverpool Q-meters [61][62]. There is one Q-meter for each species of target which needs to be monitored. The Q-meters house the resistor and capacitor used in the LRC circuit and these were located in a magnetically shielded box outside of the target. The inductor is wound around the target cells inside the cryostat. The inductor and the $\mathrm{RC}$ circuit in the Q-meter were connected by semi-rigid coaxial transmission cable, commonly referred to as "lambda-by-two" $(\lambda / 2)$ cable. The length of this cable is set to an integer number of half wavelengths of the central driving frequency of the circuit, which is the Larmour frequency of the target species being measured. The cable adds resistance 
and capacitance to the circuit, which in turn distorts the NMR signal. This distortion is zero at the central frequency if the length of this cable is restricted to an integer number of half wavelengths. Figure 3.14 shows a diagram of the major electronic components of the circuit.

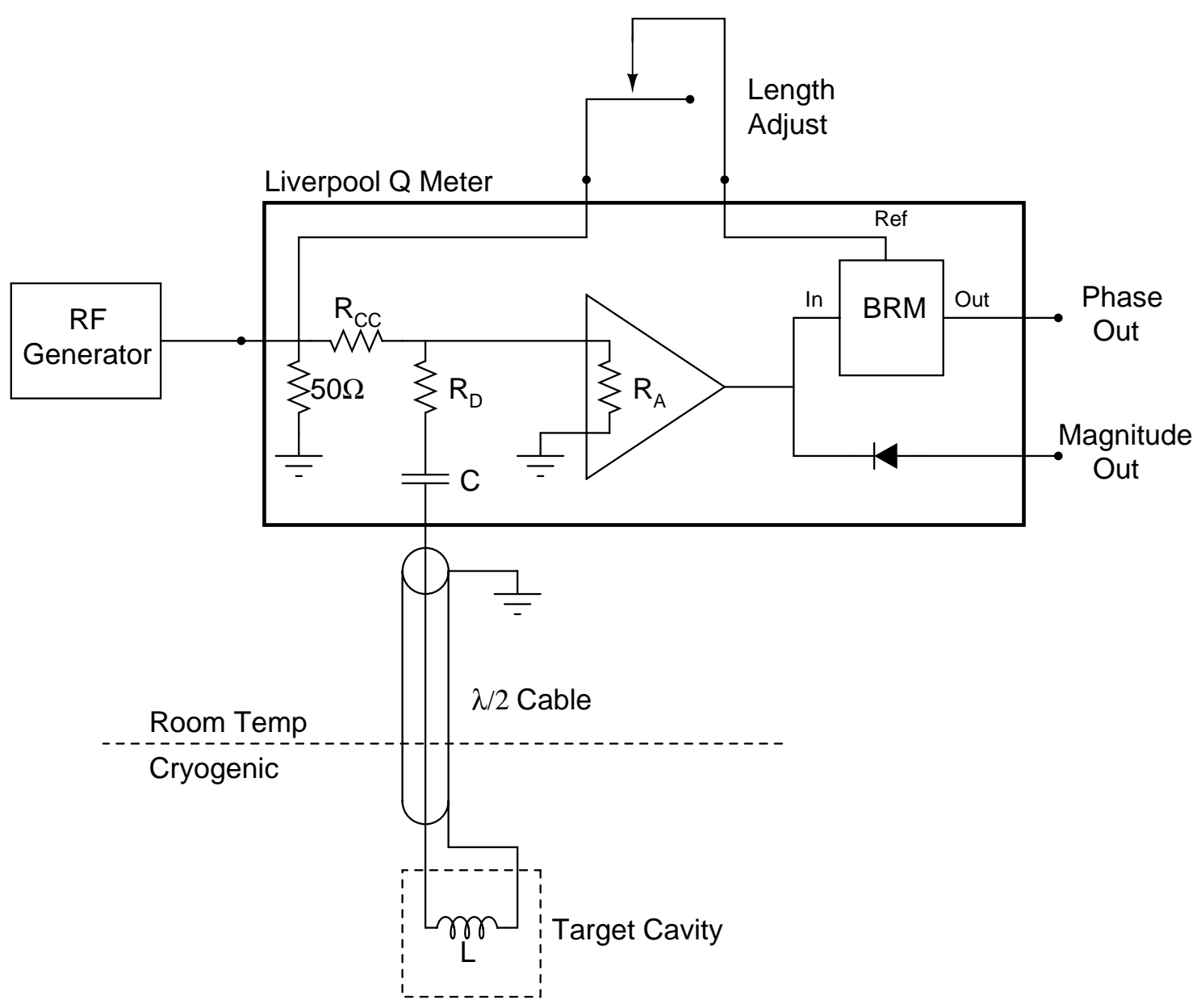

Figure 3.14: Schematic of the Liverpool Q-meter and NMR system

The primary purpose of the Q-meters is to measure the voltage across the LRC circuit as a function of frequency. If the current in the circuit is kept constant then the voltage is only a function of the total impedance of the system (V=IZ), allowing 
the extraction of the inductor impedance and magnetic susceptibility of the target. The LRC circuit is powered and driven by a Rohde and Swartz RF generator. This device supplies approximately $100 \mathrm{mV}$ of power to the Q-meter, while also sweeping the circuit through a pre-programmed set of frequencies centered around the Larmour frequency of the target. The proton target for example was swept through a cycle of 212.6 - $213.4 \mathrm{MHz}$. Inside the Q-meter housing the RF signal is split into two branches. The first feeds the series LRC circuit and $R_{A}$, a high impedance amplifier, which are arranged in parallel. $R_{A}$ has a high input impedance compared to the LRC circuit to help ensure the constant current condition. The voltage at the output of this amplifier changes with the frequency of the RF current in the system. As the frequency approaches and crosses resonance the impedance of the system drops sharply due to the absorption of energy by the target, and due to the constant current the voltage follows. The output from $R_{A}$ can be read directly from the diode output of the Q-meter. This signal still contains the dispersive part of the impedance and therefore is only used in the coarse tuning of the NMR system.

The dispersive part of the susceptibility is removed by feeding a reference signal from the RF generator as well as the amplified LRC signal into the Balanced Ring Modulator (BRM). The BRM is a phase detector, which compares the two input signals and requires the phase between them be set to zero. If $V_{A}$ is the voltage from the amplifier and $V_{I}$ is the input from the rf generator then the output from the Phase out port on the Q-meters is defined as:

$$
V_{\text {phase }}=V_{A} V_{I} \cos \phi
$$

where $\phi$ is the phase between $V_{I}$ and $V_{A}$. The term $\cos \phi$ is set to unity by adjusting 
the length of the cable from the RF generator to the BRM. This process subtracts off the imaginary part of the amplified LRC signal, thus isolating the absorptive part of the susceptibility.

The signal output from the BRM contains the desired quantity $\chi^{\prime \prime}$, as well as some multiplicative and additive constants (such as the inductance and resistance of the coil). If these constants could be easily separated out then the output signal of the BRM could be inserted into Eq. 3.6 and the polarization determined. Instead a calibration process, utilizing the thermal equilibrium (TE) signal of the material, is used to account for these extra factors. Boltzmann statistics allows the polarization of the target material to be calculated when the target is in a state of thermal equilibrium (Eq. 3.3). Using the known TE polarization, the TE signal and enhanced signal from the BRM along with Eq. 3.6 it is possible to solve for the enhanced signal polarization.

$$
\frac{P_{e n h}}{P_{T E}}=\frac{\int_{0}^{\infty}\left(A+B \chi^{\prime \prime}{ }_{e n h}\right) d \omega}{\int_{0}^{\infty}\left(A+B \chi^{\prime \prime} T_{T E}\right) d \omega}
$$

The NMR signal analysis which is required to complete the integration over the output from the BRM will be discussed in the following section.

\subsubsection{NMR signal Analysis}

The polarization of the target material is determined by integrating the output signal from the Q-meters over all frequencies. Before the integration is performed several steps are needed in order to extract and isolate a clean signal. Figure 3.15 illustrates the procedure for each of these steps.

The output signal from the BRM has a characteristic voltage vs. frequency de- 
NMR Signal Analysis

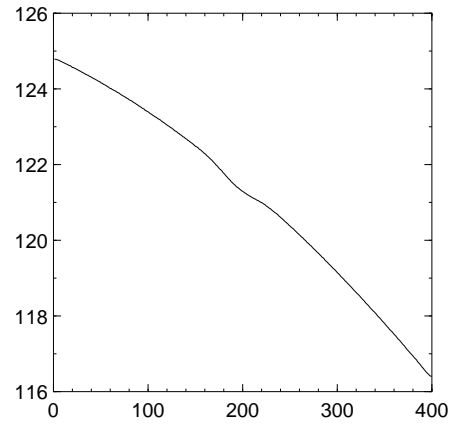

Signal is acquired by the NMR DAQ system

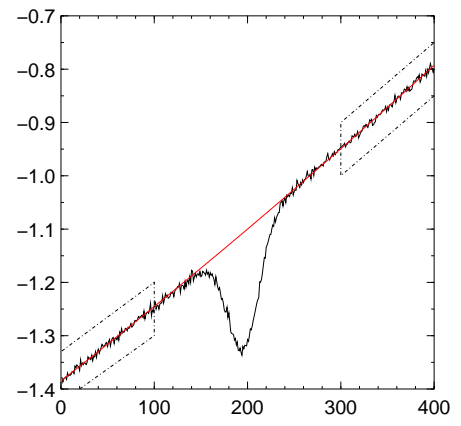

Polynomial is fit to wings of the signal

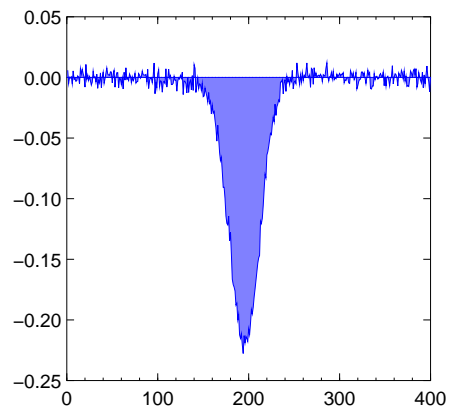

Signal is integrated, giving area

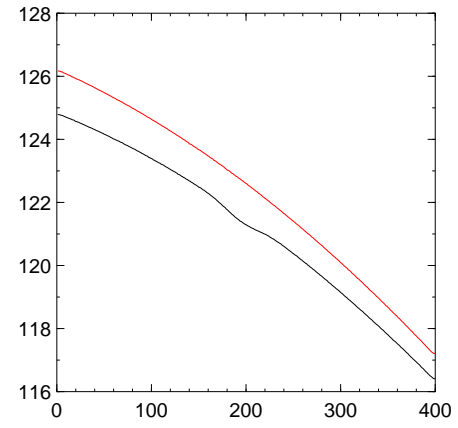

Baseline is subtracted from the signal

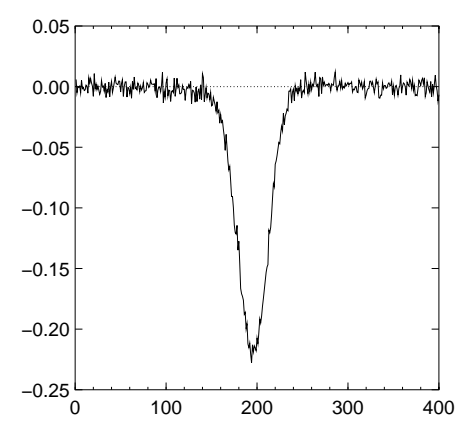

Polynomial is subtracted from the signal

Area $\times \mathrm{CC}=$ Polarization

Calibrating the NMR system gives polarization

Figure 3.15: Extraction and integration of proton absorption signal 
pendence, which reflects the "tune" of the LRC circuit. The tune of this curve reflects the resistive, capacitive and inductive components of the circuit and a typical curve is shown in the first plot of Fig. 3.15. The dip in the middle is the absorption signal from the target. In order to separate the absorption signal from the background signal a baseline measurement is taken. A baseline is an NMR signal which is taken at a magnetic field which is 1-2 \% different from the operating field. This in effect changes the Larmour frequency of the protons (deuterons) in the target material and removes any absorption signal in the observed frequency range. The second graph in Fig. 3.15 shows a baseline signal which is slightly mismatched with the NMR absorption signal. This is often due to changing resistance of the $\lambda / 2$ cable which results a change in the tune of the circuit. Once an enhanced signal is obtained the baseline curve is subtracted. After the baseline subtraction a polynomial fit is made to the edges of the signal and then subtracted. The polynomial fit, shown in the third graph of 3.15 , is necessary to compensate for inaccurate baseline subtraction. The result of these two subtractions is called is called the poly-subtracted signal, which is the fourth graph. After this second subtraction the polarized curve can be integrated. In theory the integration should be over all frequencies; in reality the signal is zero for all frequencies except for a finite width around the Larmour frequency of the polarized nucleon. Thus the integration is only over the range of frequencies swept by the Rohde and Schwartz Generator.

The final step involves multiplying the area under the curve for the enhanced NMR signal by the calibration constant, $\mathrm{C}$, is defined by the polarization and area of 
the thermal equilibrium signal (TE).

$$
\begin{gathered}
P_{e n h}=C \times \text { Area }_{e n h} \\
C=\frac{P_{T E}}{\text { Area }_{T E}}
\end{gathered}
$$

\subsubsection{Polarized Target Software}

The EG1 polarized target software was designed and maintained by a collaboration from the University of Virginia, INFN at Genova and the Jefferson Lab Target Group. The running conditions in Hall-B required that all target systems be monitored and operated remotely. To this end a two computer network was established between the hall and the counting house. A computer with an external Multiple Input/Output (MIO) board was installed in Hall-B, with the majority of the MIO channels devoted to the collection of NMR output signals. A computer in the counting house ran the Polarization Display Panel (PDP) program, a LABVIEW program designed to control the magnet as well as monitor and record the polarization signals received from the computer in the hall. The EIO tube and pump system were also remotely controlled via panels installed in the counting house. A second LABVIEW program was installed in order to monitor the temperature and level of helium in the banjo. This program also allowed shift workers to operate the stepper motors which controlled the target position as well as the run and bypass valves. The control of the helium flow from the buffer dewar into the service module was automated through the EPICs (Experimental Physics and Industrial Control System) software and therefore required very little input from the target shift worker. 


\section{Chapter 4}

\section{Data Analysis}

The calculation of $A_{\| \mid}$is the first step in extracting $g_{1}$. The path to $A_{\|}$requires the reconstruction of electron tracks, matching them to their correct helicity state and then weeding out false electron signals such as $\pi^{-}$particles and symmetric $e^{+} e^{-}$pairs. Subtraction of the ${ }^{15} \mathrm{~N}$ background, radiative corrections and determination of the beam and target polarizations are also necessary. The following chapter outlines the procedures used to construct a fully corrected $A_{||}$from raw data files.

\subsection{Data Acquisition and Storage}

During the three months of running time EG1 collected over 3 billion triggers of data. This data, initially in the form of ADC and TDC values from the various detector packages that make up CLAS, were stored on tape in BOS format. BOS is a program system written in FORTRAN 77 for the dynamic management of data. A BOS file is composed of "banks", which are subdirectories that correspond to an individual detector or detector part. These banks and their structure are identified by "headers" 
and the actual data is stored in the "body". The average run was composed of 10 million triggers and occupied 10-20 GBytes of space.

\subsection{Detector Calibration}

Each detector package in the CLAS was calibrated before track reconstruction began. The TDC offsets for each paddle of the time of flight detector were optimized to give the correct flight time values, and thus the correct $\beta$, for each particle. The electrocalorimeter photomultiplier gains were adjusted so that the PMT signal was proportional to the energy of the electron. This was achieved by comparing a GEANT simulation with the EG1 data. The drift chamber calibration fits the calculated distance-of-closest-approach (DOCA) function with the measured drift times, allowing for drift distances to be extracted.

Approximately $10 \%$ of the EG1 data set was used for these calibration procedures. The data runs used were required to have optimal detector, data acquisition, beam and target conditions. Once calibration was completed, and track coefficients optimized, the code was "frozen" and track reconstruction for the total data set could begin.

\subsection{Track Reconstruction}

The CLAS reconstruction software, RECSIS, consists of several detector analysis packages, including the polarized target and torus field maps. RECSIS performs two important tasks, track reconstruction and preliminary particle identification. Cooking refers to the processing of raw data banks by the RECSIS code. 


\subsubsection{Hit Based Tracking}

Track reconstruction is initially performed by looking at signals in each sector of the drift chamber. The signals, or wire hits, are created when a charged particle ionizes

the gas and creates a current in the sense and field wires. A library of possible track segments, generated from simulations, are used to match individual superlayer hits and then construct small sections of a track. These segments are connected into a complete particle track if there are hits in at least five superlayers. If there is only one negative track per event then this is defined to be the scattered electron. If there are two or more negative tracks in the event then the electron is defined to be the one which most closely matches the characteristic electron response in the detectors. The ideal electron candidate is one which triggers the $\breve{C}$ erenkov Counters, has the desired showering pattern in the calorimeter as well as triggering the scintillators. This procedure is called hit based tracking (HBT) and results in a momentum resolution of $3-5 \%[51]$.

\subsubsection{Start Time Determination}

This resolution is greatly improved by the use of time based tracking (TBT). Before implementing the TBT procedure it is necessary to know the time of interaction at the target, called the start time, for each scattered particle. The start time is deduced from the the time of flight detectors. Once a particle has been identified and it's momentum defined using HBT, it is possible to reconstruct the time of interaction at the vertex by subtracting the particle flight time from the TOF time, $t_{t o f}$.

$$
t_{\text {start }}=t_{t o f}-\frac{d_{\text {track }}}{c \beta}
$$


The start time accuracy is improved by applying an RF correction. The RF signal of the accelerator is recorded in the data stream. This signal, plus a constant factor due to the distance the bunch must travel to the target, defines the interaction time. Ideally the $t_{\text {start }}$ value calculated from the TOF will correspond with arrival of the RF signal. The time of flight has a finite resolution, causing the values of $t_{\text {start }}$ to be centered around the beam signal with a width corresponding to the TOF resolution of 163 ps. If the mean of the $t_{\text {start }}$ distribution is different from the time of the bunch arrival then $t_{r f}$ is added in order to align the two events in time.

$$
t_{r f}=t_{\text {start }}-t_{\text {beam }}
$$

Applying the RF correction defines the start time as:

$$
t_{\text {start }}^{\prime}=t_{\text {tof }}-\frac{d_{\text {track }}}{c \beta}+t_{r f}
$$

Plotting $t^{\prime}-t_{r f}$ should give a Gaussian distribution centered around zero. This distribution is shown in Figure 4.1.

\subsubsection{Time Based Tracking}

The start time can now be used to improve the momentum resolution of the hit based tracking procedure. The problem with HBT is that the particle most likely did not actually pass through the wire which registers a hit. In fact the particle most likely traversed at a distance, $\mathrm{d}_{d r i f t}$, from the sense wire. It is possible to calculate $d_{d r i f t}$ by finding the drift time, $t_{d r i f t}$, and using this additional information to create more 


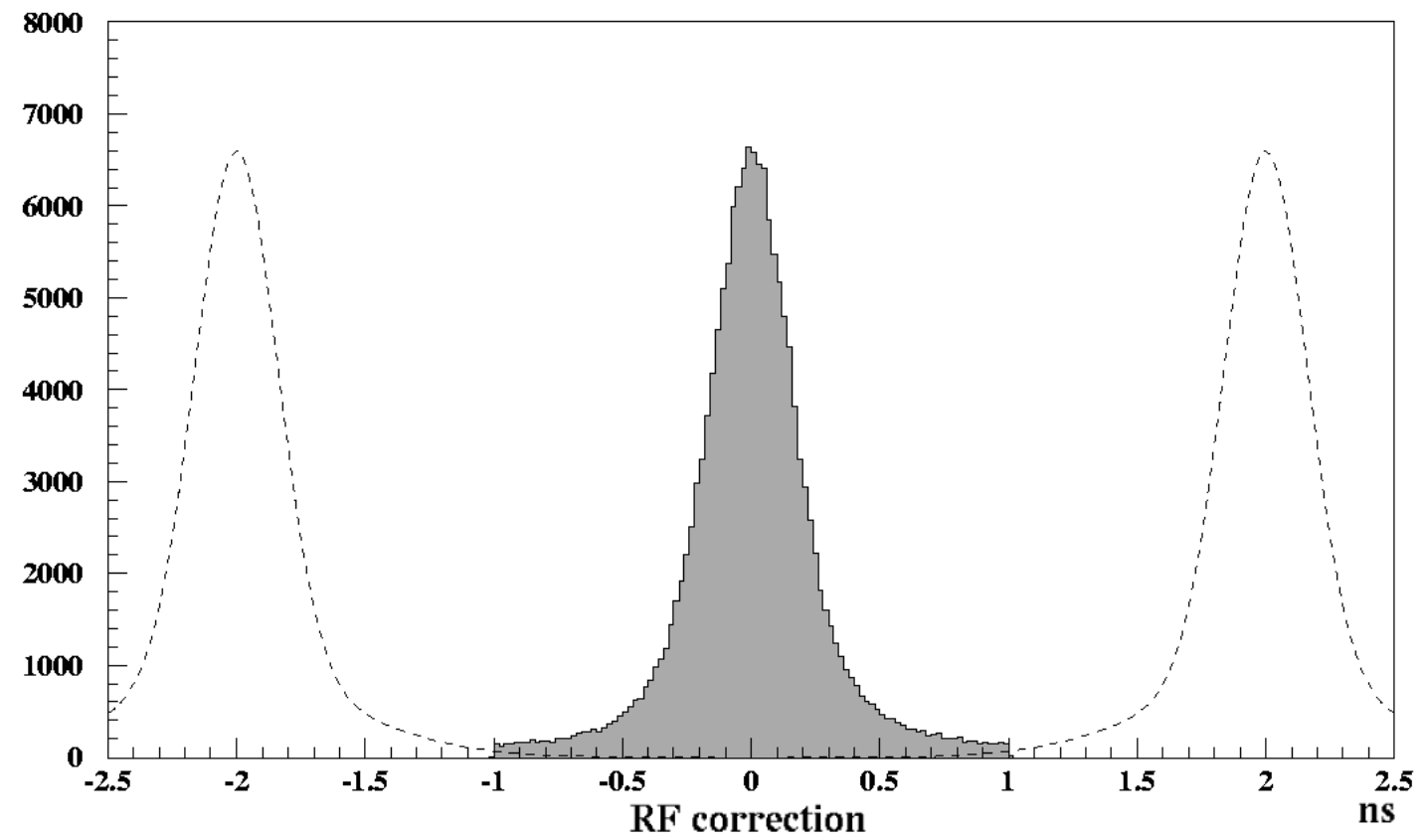

Figure 4.1: The Gaussian distribution given by $t_{\text {beam }}^{\prime}-t_{r f}$, where $t_{\text {beam }}^{\prime}$ is the rf corrected start time taken from the time of flight paddles. The preceding and following electron bunches in the beam are shown to the left and right.

accurate track and thus momentum reconstruction[51].

$$
t_{d r i f t}=-t_{t d c}+t_{\text {start }}-t_{\text {flight }}-t_{\text {prop }}+t_{0}-t_{\text {walk }}
$$

The raw time read out by the drift chamber TDC is $t_{t d c}$, the time of interaction at the vertex is $t_{\text {start }}$, the time it takes for the particle to go from the vertex to the ionization point is $t_{\text {flight }}$, the time required for signal propagation down the wire is $t_{\text {prop }}$, the time delay for the wire is $t_{0}$ and the time-walk correction made for short drift times due to different ionizations for slow and fast particles is $t_{\text {walk }}$. A table containing position dependent drift velocities, which are on the order of 20-40 10 
$\mathrm{m} / \mathrm{s}$, were used to convert $t_{\text {drift }}$ into $d_{\text {drift }}$. The drift distance only gives a radius of possible interaction around the hit wire however, resulting in ambiguity on whether the particle passed to the right or the left of the sense wire. This is resolved by choosing the track with the smallest $\chi^{2}$ value.

The path length of the particle, as determined by TBT, along with the time of flight given by the scintillators defines $\beta$, where $\beta=\frac{v}{c}$. The fastest negative track is defined as the scattered electron with a $\beta=1$. The momentum and $\beta$ of the remaining negative and positive tracks defines the mass of these particles. The average mass resolution, with time base tracking, is $0.5 \%$.

\subsection{Helicity Information and HelP Tables}

Spin asymmetries are small and require a careful accounting of electron helicity states in order to avoid systematic, helicity dependent errors. The helicity structure of the beam was tied to a "sync" signal, which is an oscillating square wave with a period of 1 second. A new electron helicity state was delivered to the hall every second. Helicity states are organized in pairs, the original and the complement. The original helicity state is chosen in a pseudo-random way at the injector. The complement state, which immediately follows the original state, is always of the opposite helicity. The helicity state which follows the complement is again chosen in a pseudo-random way and the process is repeated (see Fig. 4.2).

The helicity state of the beam is recorded in two separate banks in the BOS data files. Each bank contains a helicity bit which is either 1 or 0 , and a sync bit, also 1 or 0 , as well a helicity scaler whose value is incremented by one every time a new 
helicity state is introduced. Each of these banks is updated for every event (every second).

\section{Sync Pulse}

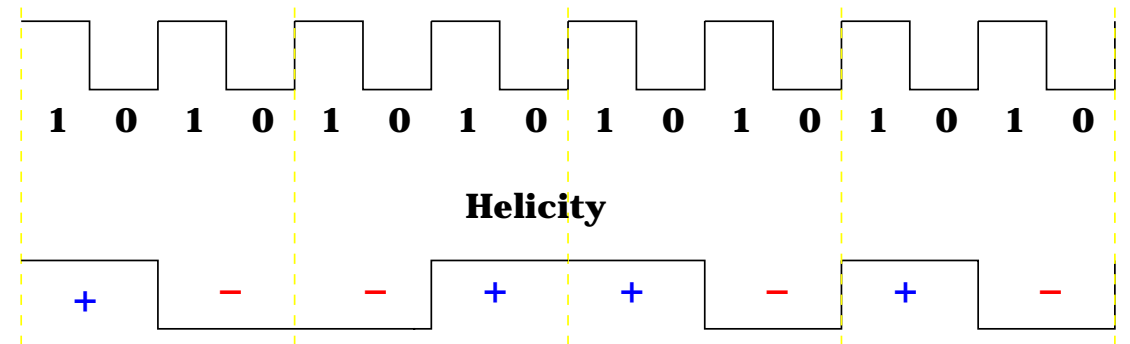

Original/ Complement State

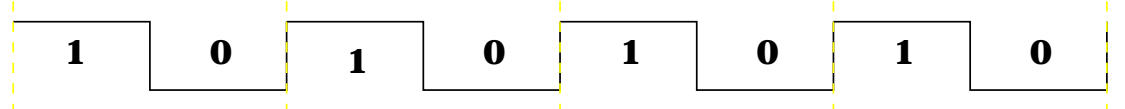

Figure 4.2: Beam helicity and sync bit configuration

It is possible for the recorded data to have broken sequences of helicity pairs, which may be due to dead time problems with the DAQ. These broken sequences result in several types of irregularities: the helicity scaler doesn't increment by one with each helicity state, one of the banks is missing an original or complement state, the Read Out Controllers (ROCS) are not in sync for the entire helicity state or the helicity states stored in the two banks disagree. Broken sequences can cause false asymmetries to occur and for this reason the HelP program was developed. The HelP program produces HelP files, which contain a flag for each helicity state. The flag is 1 for the original and 2 for the complement state. If the flag is $\leq 0$ then one of the above problems occurred and the events that occurred in that helicity pair should be thrown out. The tables contain information on the helicity of each state, as well as the 
integrated charge from the Faraday Cup and the number of electrons scattered into each sector per helicity event. These tables are used at the initial stages of analysis to remove "bad" helicity pairs.

Accurate book-keeping is not enough to remove helicity dependent false asymmetries. It is possible, and in fact the case for EG1, that there are not equal numbers of electrons in the positive and negative helicity bunches. This is easily accounted for by normalizing each helicity spectra with the total Faraday cup charge. The Faraday cup charge is read out once a second and stored in scaler memory. After five helicity pairs have been delivered (10 seconds) the memory is integrated and read out into the data stream. HelP also ensures that each helicity pair is correlated with the correct Faraday cup reading as well as placing a negative flag on runs with no Faraday cup reading. The charge asymmetry for the EG1 run was $\sim 0.3 \%$.

\subsection{DST Production}

The files produced as a result of the cooking process are large, $\sim 10 \mathrm{MB}$ of disk space for a single run. Smaller files, historically called data storage tapes (DSTs), were created in order to allow a large portion of the data set to be transfered to individual institutions for analysis. The DST files incorporated the results of the HelP Tables and included only events which passed all helicity tests. In addition a limited set of parameters in the BOS files were included in the DSTs. These parameters are only those necessary for determining kinematics, helicity and electron cuts. 


\subsection{Momentum Corrections}

The accuracy of the momentum reconstruction can be checked by the requirement that the $\mathrm{e} \rightarrow \mathrm{p}$ elastic peak in the $\mathrm{W}$ spectrum is centered around the proton mass $(0.938 \mathrm{GeV})$ for all electron scattering angles. Due to inaccuracies in the survey data of the drift chamber location and errors in the magnetic field map there is a shift in the elastic peak, the magnitude of which depends on the beam energy, torus field, $\theta$, $\phi$, and the sector of CLAS in which the electron is detected. Momentum corrections [65], can be used to align the elastic peak of all the sectors around the proton elastic peak, must be performed for all data. The momentum corrections take the following form:

$$
P_{\text {corrected }}=F(\theta, \phi) \cdot \frac{I_{0}}{I_{\text {readout }}} \cdot P_{\text {reconstructed }}
$$

where $F(\theta, \phi)$ is the correction factor, $I_{0}$ is the actual torus field, and $I_{\text {read }}$ is the torus field in the database and the one used in the track reconstruction. $F(\theta, \phi)$ take the following form:

$$
F(\theta, \phi)=\left(a+b \phi+c \phi^{2}\right) \frac{d \theta-f}{g \theta-h}
$$

The seven parameters are determined by fitting the position and minimizing the width of the elastic peak for each sector of the CLAS. The corrections to the electron

momentum are of the order of $\sim 0.1 \%$ and decreases for increasing electron polar angle. The effects of these corrections is illustrated by Figs. 4.3 and 4.4. 


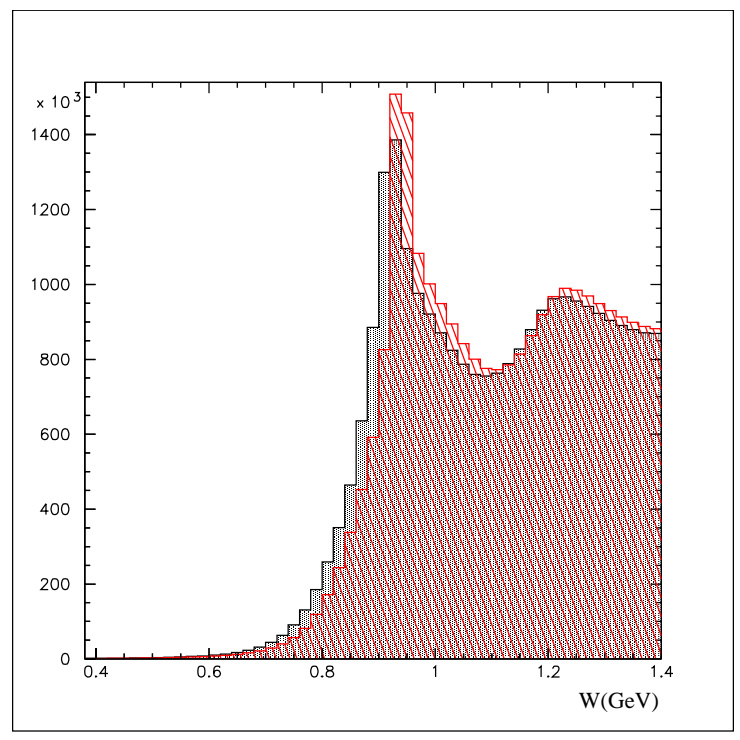

Figure 4.3: The W spectrum before (black) and after (red shaded region) momentum corrections for beam energy of $2.565 \mathrm{GeV}$ and inbending torus setting.

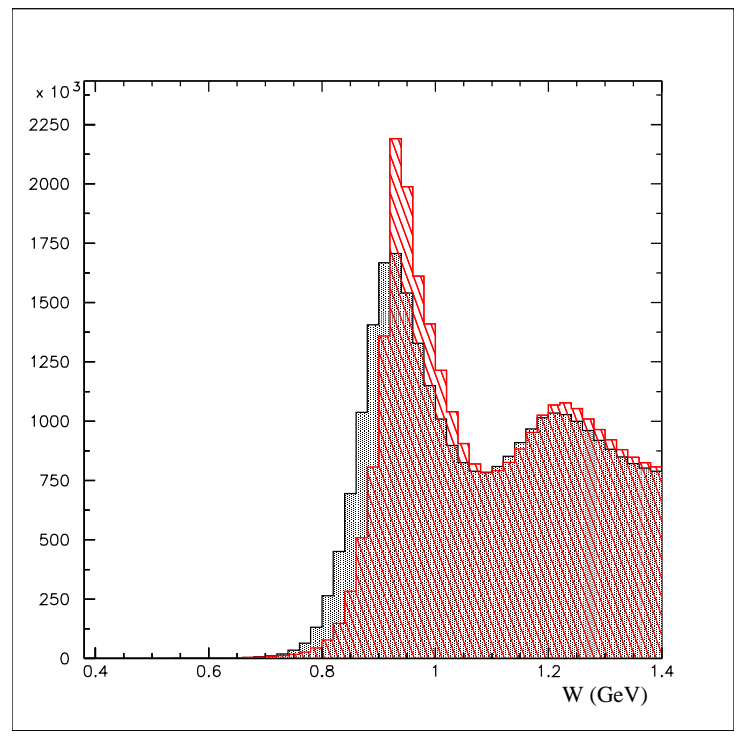

Figure 4.4: The W spectrum before (black) and after (red shaded region) momentum corrections for beam energy of $2.562 \mathrm{GeV}$ and outbending torus setting. 


\subsection{Electron Identification}

Cuts are applied to the data primarily to eliminate $\pi^{-}$particles which may have been mis-identified as electrons. Another purpose for cuts is to remove electrons which scattered from non-target material such as windows and radiation shields. It is possible to remove all background events with strong cuts, but this purity is achieved at the high price of removing many "good" electrons as well, which in turn increases the statistical error. At the other extreme, loose cuts provide the lowest statistical error, but a larger systematic error due to contamination. Optimal electron cuts lie between these two paths, where the statistical and systematic errors are minimized.

\subsubsection{Vertex Cut}

The track resolution of CLAS allows a distinction in scattering from the target cell, outer heat shield and cryostat window, see Fig. 4.5.

In practice this means that a relatively tight vertex cut can be imposed in order to remove electrons which may have scattered from materials in the cryostat other than the target cell. This cut clearly depends on the accuracy of the RECSIS track reconstruction. This resolution varies with beam energy and torus field. In order to minimize the number of electrons removed, a relatively loose cut was made. Electrons with a vertex reconstruction of $\mathrm{z}$ where, $-60 \geq z \geq-52 \mathrm{~cm}$, were discarded. The center of the target was located at approximately $-56.0 \mathrm{~cm}$ along the beamline.

Before the vertex cut was applied, a small correction was performed on the reconstructed z position of the scattered electron. Due to an unanticipated horizontal shift in the target position relative to CLAS there is a sector dependence in the track recon- 


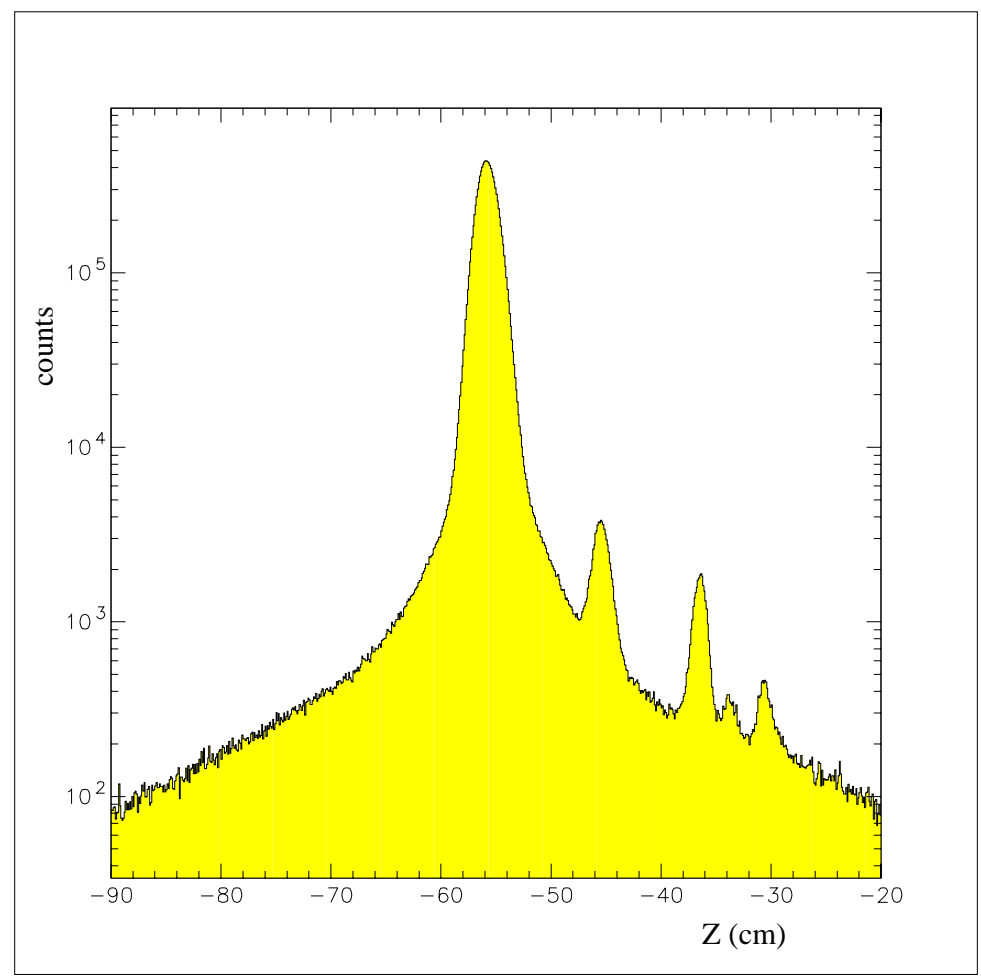

Figure 4.5: The above plot shows the $\mathrm{z}$ position of scattering along the beamline as a function of the number of electrons scattered. The central peak represents scattering from the target material as well as the target cell and banjo windows. The remaining peaks to the right represent scattering from the radiation shields and OVC exit window. 
struction. A correction [66] which removes the phi dependence of the $\mathrm{z}$ reconstruction has been applied and the results are shown in Fig 4.6.

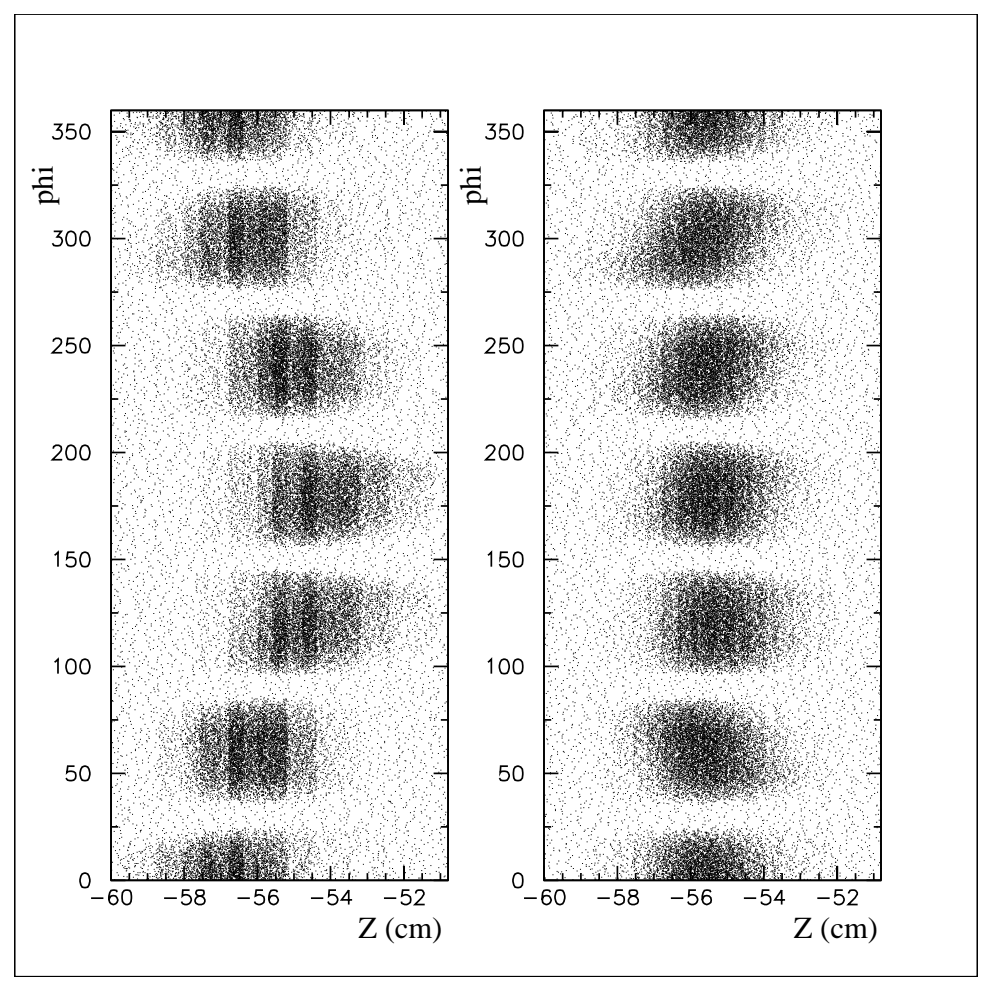

Figure 4.6: The left and right pictures show the vertex reconstruction in the $\mathrm{Z}$ direction as a function of phi before and after the vertex correction. The clusters of points show the six sectors which make up the CLAS detector.

\subsubsection{C̆}

The $\breve{C}$ erenkov Detector provides a clear separation of electrons and the majority of pions with momentum less than $2.8 \mathrm{GeV}$. Above $2.8 \mathrm{GeV}$ the velocity of the pion is high enough to emit $\breve{C}$ erenkov light, causing data with beam energies above this threshold to possibly have a higher pion contamination. In order to minimize the 
contamination of the electron sample for the $4 \mathrm{GeV}$ data the $\breve{C}$ erenkov cut rejected all electrons with a photo-electron signal less than 1.5 photo-electrons.

A study [67] of the effect of the $\breve{C}$ erenkov cut on the pion contamination determined that a cut at 1.5 photoelectrons was optimal. This study demonstrated a steady decline in the $e^{+} / e^{-}$ratio as a function of $\breve{C}$ erenkov cut. The ratio becomes cut independent at 2.0 photoelectrons, see Fig. 4.7. The reason for this behavior is that the production rates of $\pi^{+}$and $\pi^{-}$are believed to be equal. As the $\breve{C}$ erenkov cut increases pions are systematically removed from the sample and the $e^{+}$rates, which are much smaller than the $e^{-}$rates to begin with, will be reduced dramatically.

\subsubsection{Electromagnetic Calorimeter Cuts}

The calorimeter is comparable to the $\breve{C}$ erenkov detector in its ability to separate electrons and pions. As discussed in section 3.2.5, the primary energy loss mechanism for electrons in the calorimeter is through radiation. The majority of electrons are completely stopped in the EC and the amount of energy they deposit, $E_{E C}$, is a constant fraction, $\alpha$, of their momentum.

$$
E_{E C}=\alpha \times P_{e}
$$

where the sampling fraction $\alpha$ is 0.265 for the CLAS EC. Because of this characteristic behavior all electrons with a $p_{e} / E_{e}$ ratio greater than three standard deviations from 0.265 were removed (see Fig. 4.8). Pions are too heavy to have a significant bremsstralung cross-section and instead lose most of their energy through atomic electron excitation and ionization. The rate of energy loss in lead, $\mathrm{dE} / \mathrm{dx}$, for pions 
is dependent on their momentum. Pions are considered minimizing ionizing particles (MIPS) when their energy corresponds to a minimum in the $\mathrm{dE} / \mathrm{dX}$ function. The value of $\mathrm{dE} / \mathrm{dx}$ is well known for MIPS in the EC and therefore it is possible to calculate the energy, $E_{E C i n}$, that will be deposited by these pions in the inner layer of the detector. The $15 \mathrm{~cm}$ of scintillator and $3 \mathrm{~cm}$ of lead result in an EC inner signal of $\sim 0.05 \mathrm{GeV}$. For this reason all electrons with a signal less than $0.05 \mathrm{GeV}$ in the inner layer of the calorimeter were considered to be mis-identified pions and were removed.

In summary, the pion contamination was minimized by removing electrons which fulfill the following conditions:

$$
\begin{gathered}
E_{E C i n} \leq 0.05 \mathrm{GeV} \\
E_{E C}<0.265\left(-0.05+P_{e}-0.3 \sqrt{P_{e}}\right) \\
E_{E C}>0.265\left(+0.05+P_{e}+0.3 \sqrt{P_{e}}\right)
\end{gathered}
$$

The total energy deposited in the calorimeter is defined two different ways. The first way, $E_{E C}$, is one in which the track reconstruction treats the calorimeter as one detector and fits the track accordingly. The second definition, $E_{E \text { Cin }}+E_{E C o u t}$, fits tracks to the inner and outer calorimeter separately. It is possible that a signal in $E_{E C o u t}$ does not exceed the threshold and therefore no track is fitted, whereas the same track may be fitted to the outer if the inner is taken into account. This fitting anomaly required $E_{E C}$ to be defined as the maximum $E_{E C}$ and $E_{E C i n}+E_{E C o u t}$.

$$
E_{E C}=\max \left(E_{E C}, E_{E C i n}+E_{E C o u t}\right)
$$




\subsection{Fiducial Cuts}

The asymmetry analysis has the advantage of not being as dependent on detector acceptance and efficiency as other types of analysis (such as cross-section calculations). The asymmetry analysis does require however that the carbon runs, which are used for background subtraction, have the same acceptance as the ammonia runs. The carbon data are in general taken in the middle of the ammonia runs and therefore this qualification is usually met. This was verified by looking at the $\mathrm{W}$ spectra of carbon and ammonia for each $Q^{2}$ bin and sector and checking that the shape was similar. The number of carbon runs is limited for EG1 and the outbending data seem to suffer from a different acceptance for the carbon and ammonia runs. The majority of the problems occur in sector five, and for that reason all data from sector five has been excluded from the outbending analysis. In addition to removing sector five, fiducial cuts have been applied to the $2 \mathrm{GeV}$ outbending data. This setting extends to low $Q^{2}$ and therefore very small scattering angles. These small angles, $\sim 8-10^{\circ}$, are at the limits of the detector packages and it is possible that electrons detected at this angle have undergone multiple scattering. Fiducial cuts [68] have been developed which cut out all electrons that scatter into edges of the detectors. These cuts do not reject a significant amount of data because they do not require scattering into regions of constant efficiency.

\subsection{Quality Checks}

Several types of quality checks were performed on a run by run basis. These checks were designed to remove runs which deviate too greatly from the rest of the sample. 
Checks which test the integrity of an entire data setting were also implemented. These checks will be discussed in the following sections.

\subsection{1 ${ }^{15} \mathrm{NH}_{3}$ Elastic Asymmetry}

The elastic asymmetry for the ammonia is plotted as a function of run in Fig. 4.9. This check is designed to make sure that runs are labeled with the correct sign for the target polarization. Although the elastic asymmetry for $\mathrm{NH}_{3}$ is small it is possible to detect a change of sign or a systematic problem.

\subsubsection{Beam Charge Asymmetry}

The beam charge asymmetry is plotted as a function of run in Fig. 4.9. The beam charge asymmetry for EG1 should be $\sim 0.3 \%$. Runs with large deviations from this value were discarded because they indicate a possible problem with the helicity scalers in the DAQ or in the HelP Table.

\subsubsection{Detector Stability}

Two types of checks were used to test the stability of the detector over a run period. The number of particles per event and the the ratio of elastic to inelastic events is plotted in the bottom graphs of Fig. 4.9. Again any strong deviation from the average was discarded to avoid systematics to detector or DAQ stability. 

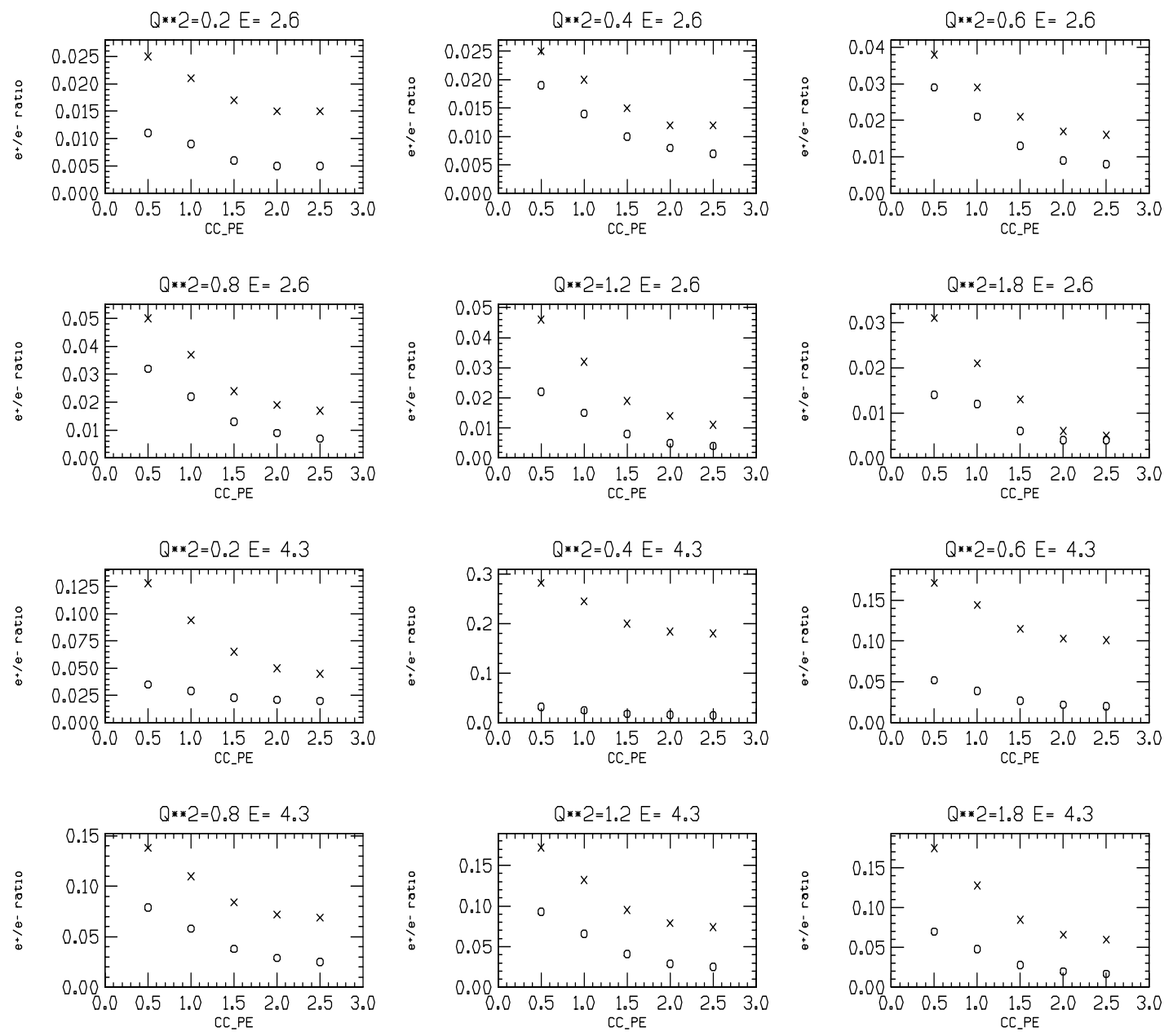

Figure 4.7: The $e^{+} / e^{-}$ratio is plotted as a function of $\breve{C}$ erenkov cut for several beam and torus settings and integrated for all $\mathrm{W}>1.5 \mathrm{GeV}$. The circles are inbending torus setting and crosses are for outbending runs. 


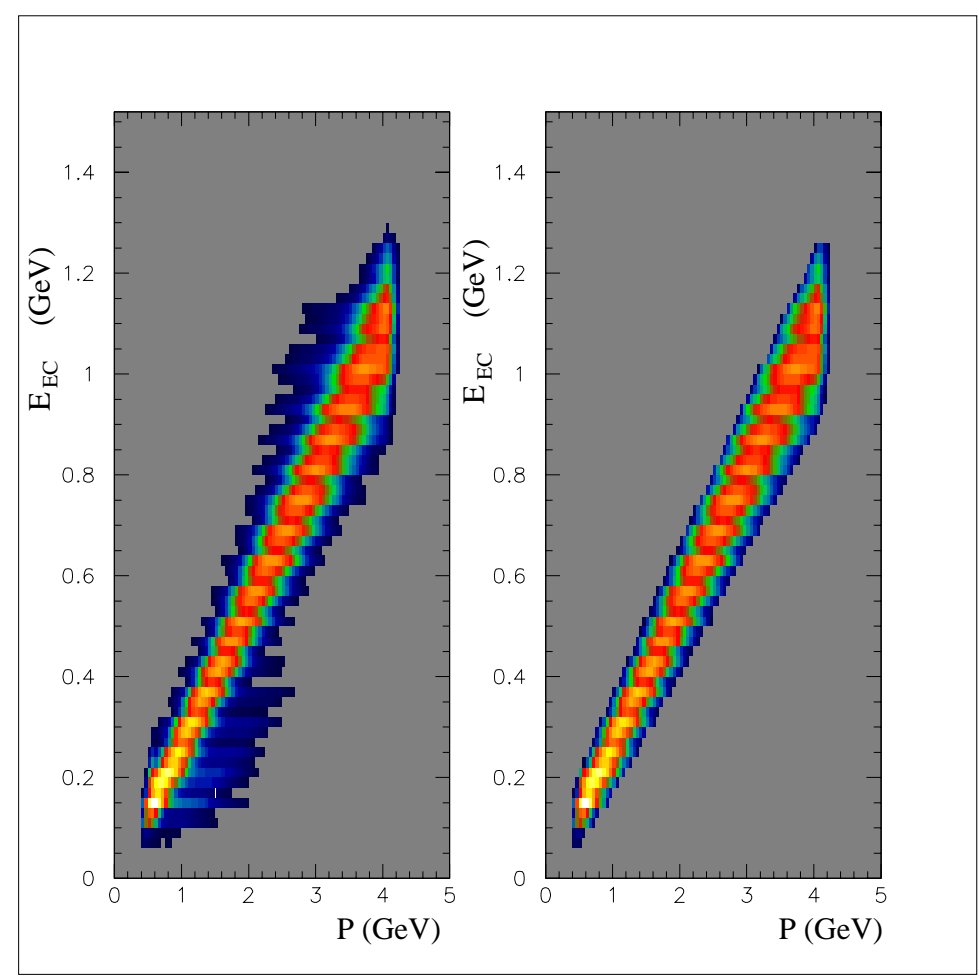

Figure 4.8: The above plots of the total energy deposited in the calorimeter versus the momentum of the particles show the distribution before and after the three sigma cut for the $4 \mathrm{GeV}$ beam setting. 


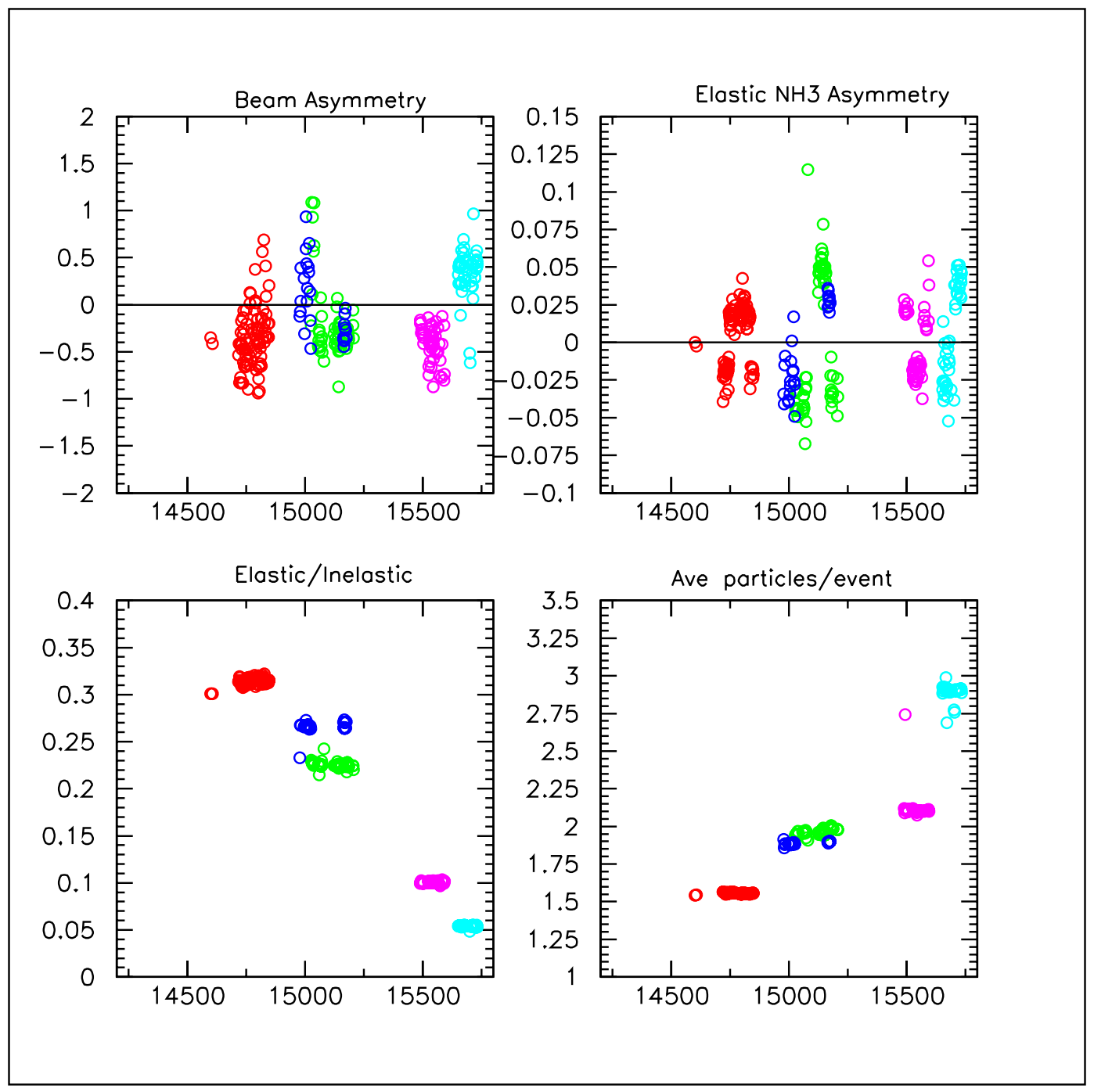

Figure 4.9: Graphs showing the quality checks on a run by run basis. The red dots correspond to $2 \mathrm{GeV}$ outbending data. Dark blue and green dots are $2 \mathrm{GeV}$ inbending, violet is $4 \mathrm{GeV}$ outbending and light blue represents $4 \mathrm{GeV}$ inbending runs. 


\subsection{4 $C_{12}$ and $N H_{3}$ asymmetry below elastic peak}

The asymmetry at $\mathrm{W}$ values below the elastic peak should be consistent with zero for both the carbon and ammonia spectra. The small polarization of the ${ }^{15} \mathrm{~N}$ will result in asymmetries less than the statistical accuracy in that kinematic region. The carbon and nitrogen spectra for each beam and torus setting were checked to ensure that the asymmetry below the elastic peak was consistent with zero. This also permitted a secondary check on the beam charge normalization.

\subsection{5 $C_{12}$ and $\mathrm{NH}_{3}$ Fiducial Regions}

The fiducial regions for the carbon and ammonia were checked by looking at the normalized total $\mathrm{W}$ spectra for each $Q^{2}$ bin and sector. If a part of the detector changed in the time between ammonia and carbon settings one would expect a change in the shape of the W spectra. Performing this check showed that all settings have the same shape in $\mathrm{W}$ for the carbon and ammonia except for the outbending settings. As discussed in the previous section this data showed different spectra for sector five as well as for the lower $Q^{2}$ regions for the $2 \mathrm{GeV}$ data. In order to correct for these differences, fiducial cuts were applied to the $2 \mathrm{GeV}$ setting and sector five was eliminated totally from all outbending runs.

\subsection{Models}

Every experiment builds on previous experiments and it is often necessary to use results from other areas in the field in order to extract new and improved quantities. EG1 is no exception to this and the following sections will note and discuss in 
detail the quantities that were not measured during this run and therefore had to be estimated or parametrized from previous experiments. Specifically the following structure functions and cross-sections were not measured: $\mathrm{R}, F_{1}^{p}, F_{1}^{n}, A_{2}$, the deep inelastic contribution to $g_{1}$ as well as the elastic form factors $G_{E P}$ and $G_{M P}$. In order to continue with the extraction of spin asymmetries it was necessary to use a model for these quantities. A set of subroutines originally used for SLAC experiment E155 radiative corrections [69] has been modified by incorporating the world data for $\mathrm{R}$, $F_{1}, F_{2}, g_{1}$, and $g_{2}$ at their corresponding $\mathrm{x}$ and $Q^{2}$ [70]. The program, "Models", fits this world data with smooth functions that can be used to predict values for inclusive cross-sections and asymmetries at all $\mathrm{x}$ and $Q^{2}$. Some of these cross-sections are measured more precisely than others and thus a combination of data and theoretical limits must be used to constrain the fits. $A_{2}$ in particular is not well know experimentally and yet it is necessary to define it for the resonance region if $A_{1}$ and $g_{1}$ are to be extracted. Theoretical constraints such as the Soffer [71] positivity limit, the Burkhardt-Cottingham Sum Rule [72] and results from MAID2000 provide guidelines for $A_{2}$. The results of the model for $A_{2}^{P}$ are shown in Fig. 4.10. The inclusion of preliminary $A_{1}$ results from EG1 for the region $W>2$ enables the models to be run iteratively, incorporating new $A_{1}$ results with each iteration. These models are also used in the calculation of the radiative corrections.

\subsection{Background subtraction}

The inclusive analysis requires that scattering contributions from the ${ }^{15} \mathrm{~N}$, helium, target cup, foils and windows be removed. The resolution of the CLAS detector 


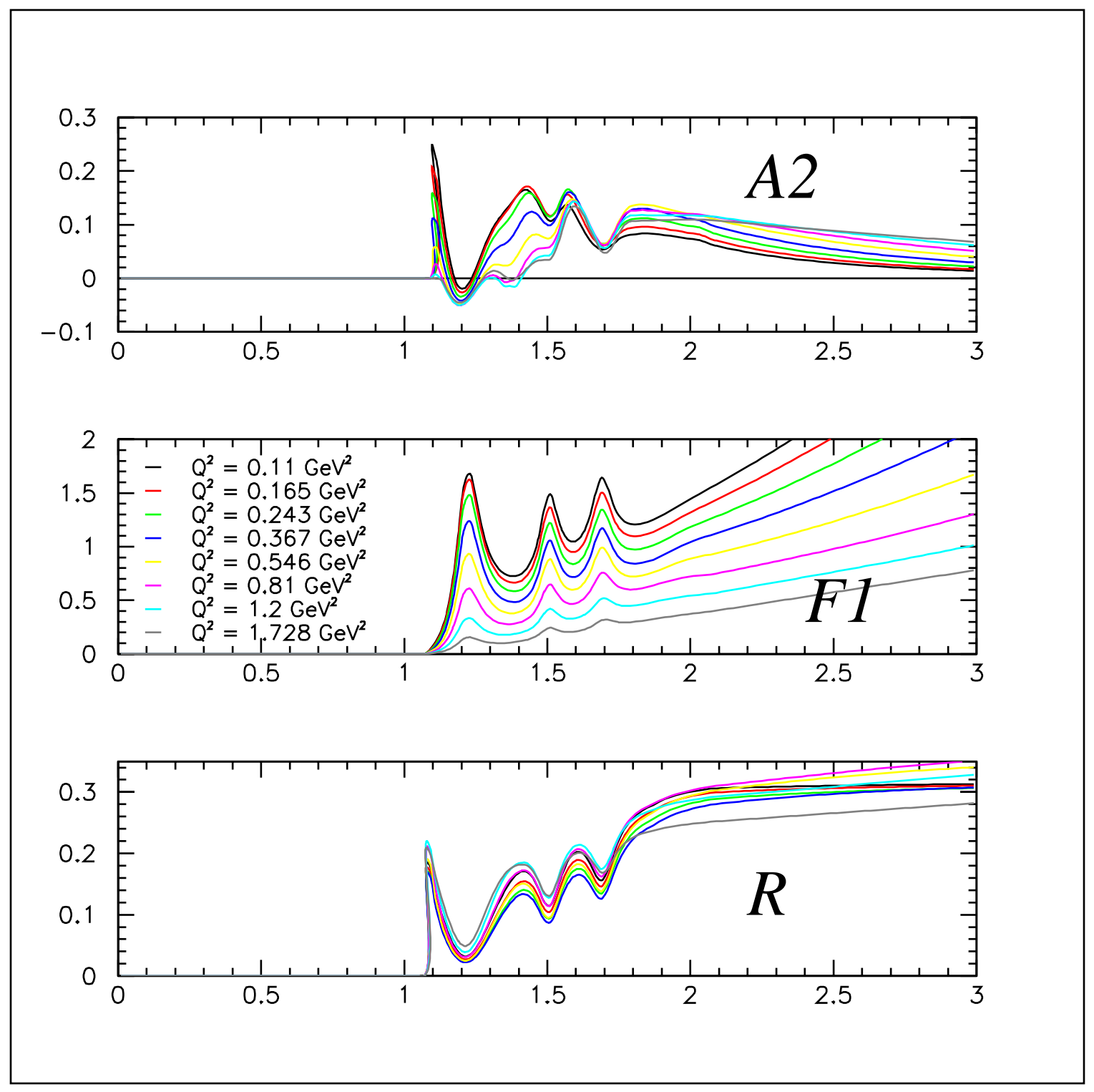

Figure 4.10: Structure functions $A_{2}, F_{1}$ and $\mathrm{R}$ plotted as a function of invariant mass and $Q^{2}$. These curves were produced by "Models" and used directly in the extraction of spin asymmetry $A_{1}$ and structure function $g_{1}$. 
limits the effectiveness of the vertex cut, which only removes scattering contributions from the external heat shields and cryostat windows. The remaining background is subtracted by using the carbon target spectra to simulate the helium, windows and 15 in the ammonia target.

Devising an accurate model of the background contributions requires precise knowledge of the cross-sections for ${ }^{15} N$, helium, and kapton for all beam energies and $Q^{2}$ ranges. Another possibility is to take data on a material that is close in structure to ${ }^{15} \mathrm{~N}$, such as ${ }^{12} \mathrm{C}$. To this end the carbon target was constructed to mimic the $\mathrm{NH}_{3}$ target as closely as possible. The external foil and window thicknesses and total radiation length are similar. The carbon target was not polarized and so it is possible to use this data to simulate scattering from ${ }^{15} \mathrm{~N}$ as well as for systematic checks on beam helicity asymmetries.

Scattering from ${ }^{12} \mathrm{C}$ and ${ }^{15} \mathrm{~N}$ is not expected to be identical. The difference in atomic number is easily accounted for but the unknown contribution from the extra neutron in ${ }^{15} \mathrm{~N}$ is difficult to simulate. There was also more helium in the carbon target due to fact that it is more dense than ammonia and therefore the target must be thinner in order to preserve comparable radiation thickness. There were no data on electron scattering from ${ }^{15} \mathrm{~N}$ as the first world measurement was only taken in January 2001 during the EG2000 run. Two different methods have been proposed in order to use carbon to simulate ${ }^{15} \mathrm{~N}$. The first method normalizes the ${ }^{12} \mathrm{C}$ and $\mathrm{NH}_{3}$ data in the region of $\mathrm{W}$ below the elastic peak. This procedure is reasonable because it is in essence normalizing the quasi-elastic peaks of each spectra, which should only differ to first order by the difference in atomic number. The problem with this method is that there are limited statistics in this region, especially for the carbon runs, and 
therefore the normalization has a large error associated with it. This method also has a large and unexplainable $Q^{2}$ dependence, up to $30 \%$, for some beam and torus settings. Likewise the dilution factor extracted using this method of normalization is highly dependent on the beam and torus setting. Although the ${ }^{15} \mathrm{~N}$ scattering data cannot be used for the EG1 analysis it was possible to see how this method of carbon normalization compares with ${ }^{15} \mathrm{~N}$ rates. This comparison revealed that the elastic peak is simulated very well but that in the inelastic region the normalized ${ }^{12} C$ falls short of the ${ }^{15} N$, see Fig 4.11. This results in an underestimated background and subsequently suppressed asymmetries.

The second proposed method for using ${ }^{12} \mathrm{C}$ to simulate ${ }^{15} \mathrm{~N}$ is also the method used in the final analysis presented in this thesis. This method uses the density and thickness of all target materials as well as the unpolarized structure functions $F_{1}^{n}, F_{1}^{p}$ and $G_{M P}, G_{E P}$ to modify the carbon spectra to look like ${ }^{15} N$. The charge normalized scattering rates, $N_{\text {target }}$, from each target are determined by the effective target thickness, $\chi_{\text {target }}$, density, $\rho_{\text {target }}$, cross-section, $\sigma_{\text {target }}$ and atomic number, $A_{\text {target }}$, of each material in the beam.

$$
\begin{gathered}
N_{N H_{3}}=\frac{N_{A}}{e}\left[\frac{\rho_{N H_{3}} \chi_{N H_{3}} \sigma_{N H_{3}}}{A_{N H_{3}}}+\frac{\rho_{H e}\left(L-\chi_{N H_{3}}\right) \sigma_{H e}}{A_{H e}}+\frac{\rho_{A l} \chi_{A l} \sigma_{A l}}{A_{A l}}+\frac{\rho_{K} \chi_{K} \sigma_{K}}{A_{K}}\right] \\
N_{C_{12}}=\frac{N_{A}}{e}\left[\frac{\rho_{C_{12}} \chi_{C_{12}} \sigma_{C_{12}}}{A_{C_{12}}}+\frac{\rho_{H e}\left(L-\chi_{C_{12}}\right) \sigma_{H e}}{A_{H e}}+\frac{\rho_{A l} \chi_{A l} \sigma_{A l}}{A_{A l}}+\frac{\rho_{K} \chi_{K} \sigma_{K}}{A_{K}}\right]
\end{gathered}
$$

$L=1.5 \mathrm{~cm}$ is the physical length of all the target cells and is defined as the distance between the banjo entrance and exit window. $N_{A}$ is Avagadro's number and $\mathrm{e}$ is the electron charge. The unknown quantities in expressions 4.12 and 4.13 are $\chi_{N_{3}}$, which describes the amount of $\mathrm{NH}_{3}$ in the $1.0 \mathrm{~cm}$ long proton target, and 


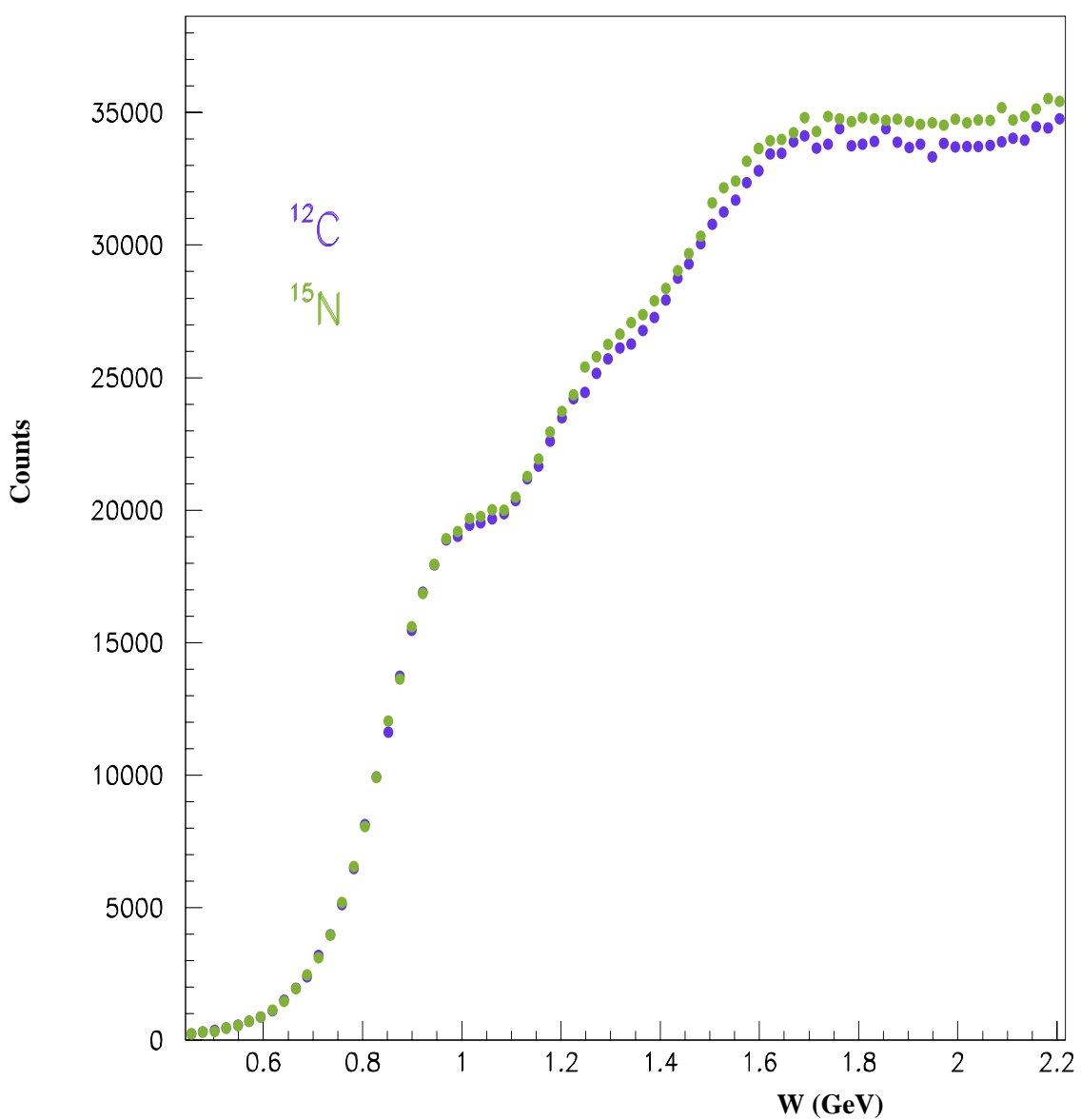

Figure 4.11: The figure above compares the ${ }^{15} \mathrm{~N}$ W spectra to the carbon spectra, which has been normalized to the ammonia spectra below the elastic peak. The normalized carbon spectra matches the ${ }^{15} \mathrm{~N}$ closely in the elastic region but a noticeable discrepancy exits for the higher $\mathrm{W}$ region. 
the cross-sections for aluminum, kapton, $\mathrm{NH}_{3}, C_{12}$ and helium. The effective length for the carbon target is well known since it is a solid disk, whereas the ammonia is composed of tiny beads which cannot fill up the entire space of the target cell completely. These cross-sections can be expressed in terms of the composite number of proton and neutrons in the material. For example:

$$
\frac{\sigma_{N H_{3}}}{A_{N H_{3}}}=\frac{8 \sigma_{N}+10 \sigma_{P}}{18}=\frac{\sigma_{P}\left(4 \sigma_{N / P}+5\right)}{9}
$$

It is possible to express all the cross-sections in this way:

$$
\frac{\sigma_{C_{12}}}{A_{C_{12}}}=\frac{\sigma_{H e}}{A_{H e}}=\frac{\sigma_{K}}{A_{K}}=\frac{\sigma_{A l}}{A_{A l}}=\frac{\sigma_{P}\left(\sigma_{N / P}+1\right)}{2}
$$

Substituting expressions 4.14 and 4.15 into 4.12 and 4.13 and then dividing the latter leaves only $\chi_{N H_{3}}$ and $\sigma_{N / P}$ as the unknowns. The ratio of the neutron to proton cross-sections can be determined by the ratio of the unpolarized structure functions $F_{1}^{N} / F_{1}^{P}$ in the inelastic region. In the elastic region the ratios of the Rosenbluth crosssections for proton and neutron are used. The parameterizations for these structure functions and form factors were taken from "Models". One can solve for $\chi_{N_{H}}$, which should be constant over the total W spectra. This is shown in Fig. 4.12 to be true only in the inelastic region, specifically at $\mathrm{W}>1.4 \mathrm{GeV}$.

The fluctuations are due to the fact that the bound and free protons in ${ }^{15} \mathrm{NH}_{3}$ have been treated exactly the same in the target model described above. The assumption is not valid in the elastic and $\triangle$ regions, but should be rather good in the higher $\mathrm{W}$ regions where many overlapping resonances make the spectra of both the free and bounds proton much smoother. The value for $\chi_{N_{H}}$ is taken from a fit to the W region above $1.4 \mathrm{GeV}$. This value varies only by $\sim 0.1 \%$ with $Q^{2}$. 


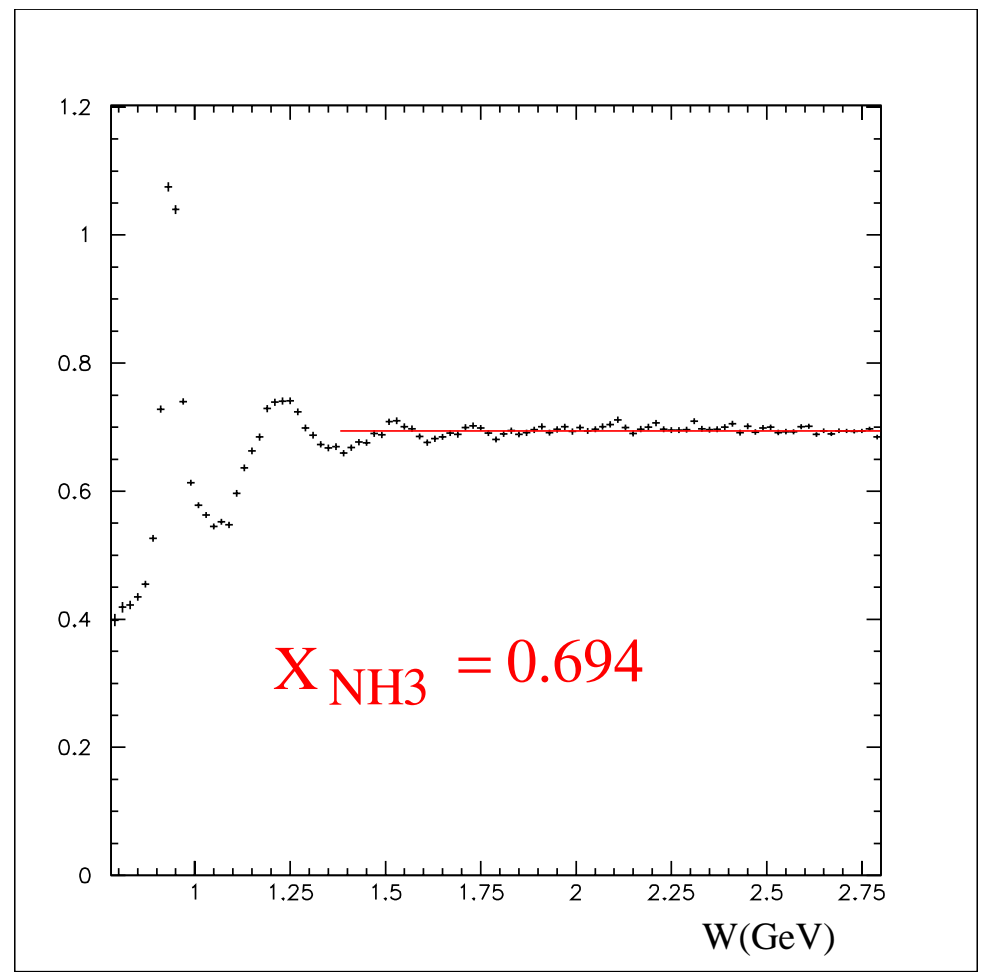

Figure 4.12: The packing fraction for $4 \mathrm{GeV}$ outbending data plotted as a function of the invariant mass, W. The red line shows the accuracy of the fit to $\mathrm{W}>1.4 \mathrm{GeV}$. 
The $C_{12}$ spectra and $\chi_{N_{H}}$ were used to simulate the ${ }^{15} \mathrm{~N}$ spectra:

$$
N_{15 N}=\frac{N_{C_{12}}\left[\left(\frac{7+8 \sigma_{N / P}}{9+9 \sigma_{N / P}}\right) \rho_{N H_{3}} \chi_{N H_{3}}+\rho_{H e}\left(L-\chi_{N H_{3}}\right)+\rho_{A l} \chi_{A l}+\rho_{K} \chi_{K}\right]}{\rho_{C_{12}} \chi_{C_{12}}+\rho_{H e}\left(L-\chi_{C_{12}}\right)+\rho_{A l} \chi_{A l}+\rho_{K} \chi_{K}}
$$

The resulting ${ }^{15} N$ spectra was subtracted from the sum of the positive and negative helicity $\mathrm{NH}_{3}$ spectra to obtain the denominator of the proton asymmetry.

$$
A_{P}=\frac{N_{N H_{3}}^{+}-N_{N H_{3}}^{-}}{N_{N H_{3}}^{+}+N_{N H_{3}}^{-}-{ }^{15} N}
$$

Table 4.1 shows the packing fraction and dilution factor for each beam energy and magnet setting. This method is consistent across settings and ensures a good simulation of the ${ }^{15} \mathrm{~N}$ in the inelastic region where the physics asymmetries are extracted.

\subsection{Beam and Target Polarization}

The proton asymmetry is normalized to the product of the beam and target polarizations. In most polarized target experiments the polarization is measured using NMR techniques, but in EG1 the NMR coils are located outside the target cell and therefore are more sensitive to the outermost layer of the target material. The EG1 raster did not cover the entire target, so that this sensitive outer layer was not exposed to the beam and may have had a much higher polarization than the inner most ammonia beads. For these reasons the NMR was used primarily as a monitoring tool. The beam polarization was measured independently by the Møller Polarimeter in Hall-B and by the Mott detector in the injector region of the accelerator. The average beam 
Table 4.1: Average Dilution Factor and Packing Fraction in region of $\mathrm{W}>1.4$ for all Beam, Target, and Torus Settings

\begin{tabular}{|c|c|c|c|c|}
\hline Beam $(\mathrm{GeV})$ & Torus $(\mathrm{A})$ & Target Pol & DF $(\%)$ & PF $(\mathrm{cm})$ \\
\hline \hline 2.562 & -1500 & plus & $14.2 \pm 0.07$ & $0.66 \pm 0.0008$ \\
\hline 2.562 & -1500 & minus & $14.2 \pm 0.08$ & $0.65 \pm 0.0008$ \\
\hline 2.565 & 2250 & plus & $14.6 \pm 0.075$ & $0.71 \pm 0.0009$ \\
\hline 2.565 & 2250 & minus & $14.7 \pm 0.075$ & $0.72 \pm 0.0009$ \\
\hline 2.565 & 1500 & plus & $14.3 \pm 0.106$ & $0.68 \pm 0.0012$ \\
\hline 2.565 & 1500 & minus & $14.1 \pm 0.106$ & $0.65 \pm 0.0011$ \\
\hline 4.278 & 2250 & plus & $14.3 \pm 0.127$ & $0.60 \pm 0.0012$ \\
\hline 4.278 & 2250 & minus & $14.2 \pm 0.130$ & $0.58 \pm 0.0012$ \\
\hline 4.278 & -2250 & plus & $14.0 \pm 0.58$ & $0.65 \pm 0.00062$ \\
\hline 4.278 & -2250 & minus & $14.4 \pm 0.58$ & $0.71 \pm 0.00069$ \\
\hline
\end{tabular}




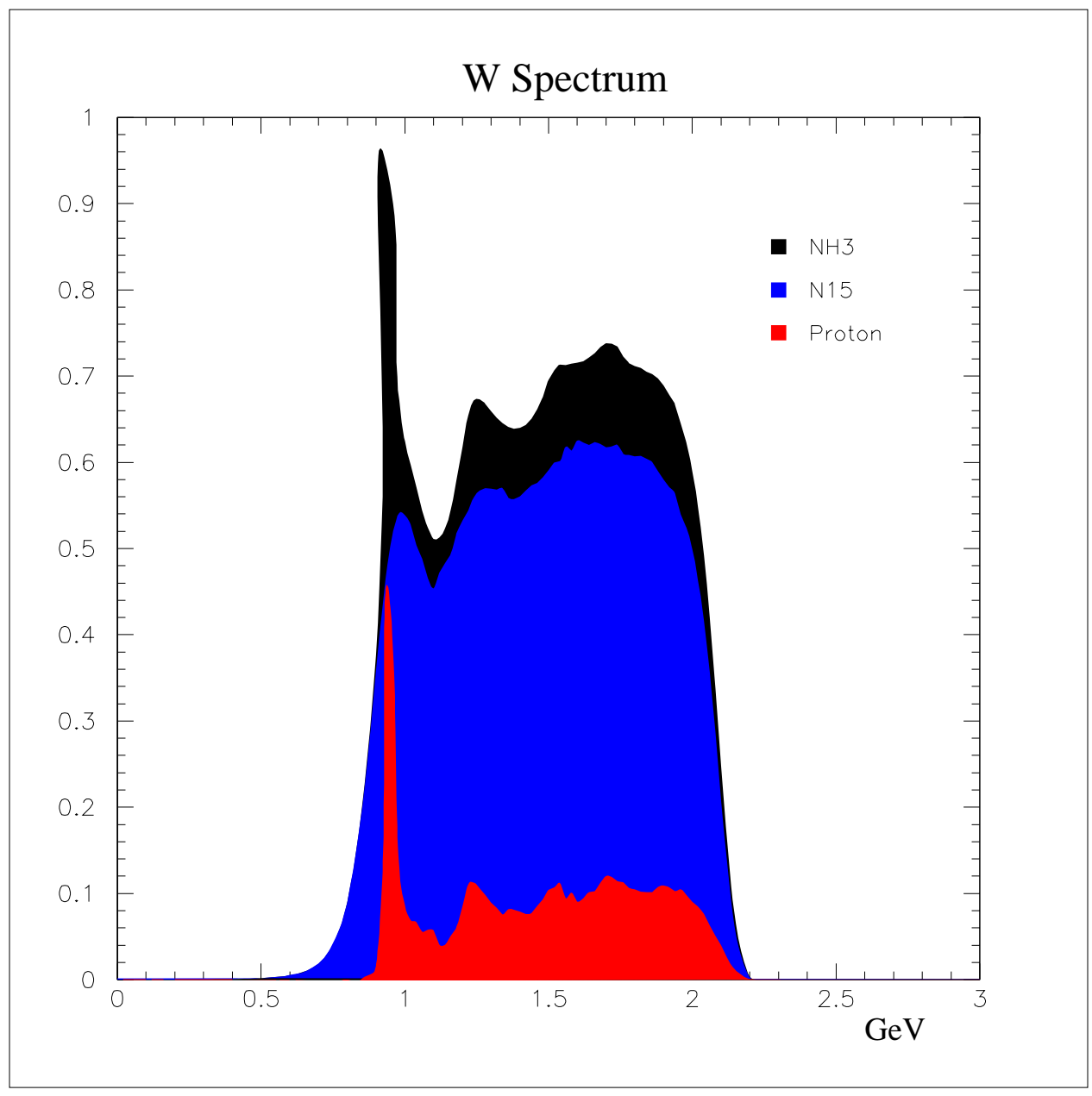

Figure 4.13: ${ }^{15} \mathrm{NH}_{3},{ }^{15} \mathrm{~N}$ and proton invariant mass spectra for the $2 \mathrm{GeV}$ inbending data. 
polarization for the EG1 run was $~ 70 \%$.

Only the product of the beam and target polarization is needed for the asymmetry correction and this can be determined directly by comparing the measured elastic asymmetry to the known values. This product is found by dividing the experimental elastic asymmetry, $A_{\text {elas }}^{\text {data }}$, by the expected elastic asymmetry, $A_{\text {elas }}^{\text {theo }}$ :

$$
P_{\text {beam }} P_{\text {target }}=\frac{A_{\text {elas }}^{\text {data }}}{A_{\text {elas }}^{\text {theo }}}
$$

The expected asymmetry is calculated from kinematics and the known values of the magnetic and electric form factors $G_{m}^{p}$ and $G_{E}^{p}$. $A_{\text {elas }}^{\text {theo }}$ is calculated as follows:

$$
A_{\text {elas }}^{\text {theo }}=D_{1} \cos \theta_{\gamma}+D_{2} \sin \theta_{\gamma}
$$

where $\theta_{\gamma}$ is defined as the angle between the incoming virtual photon and the beam direction. The asymmetries $D_{1}$ and $D_{2}$ are defined by the following equations, in which $\theta$ is the electron scattering angle and $q^{2}=Q^{2}+\nu^{2}$.

$$
\begin{gathered}
D_{1}=\frac{2 \psi v_{T}^{\prime}}{v_{L}(1+\psi) \cdot\left(G_{E}^{p} / G_{M}^{p}\right)^{2}+2 \psi v_{T}} \\
D_{2}=\frac{2 \sqrt{2 \psi(1+\psi)} \cdot\left(G_{E}^{p} / G_{M}^{p}\right) v_{T L}^{\prime}}{v_{L}(1+\psi) \cdot\left(G_{E}^{p} / G_{M}^{p}\right)^{2}+2 \psi\left(v_{T}\right)} \\
\psi=\frac{Q^{2}}{4 M_{p}^{2}} \\
v_{L}=\left(\frac{Q^{2}}{q^{2}}\right)^{2}
\end{gathered}
$$




$$
\begin{gathered}
v_{T}=\frac{Q^{2}}{2 q^{2}}+\tan ^{2} \frac{\theta}{2} \\
v_{T}^{\prime}=\tan \frac{\theta}{2} \sqrt{\tan ^{2} \frac{\theta}{2}+\frac{Q^{2}}{q^{2}}} \\
v_{T L}^{\prime}=-\frac{Q^{2} \tan \frac{\theta}{2}}{2 q^{2}}
\end{gathered}
$$

$A_{\text {elas }}^{\text {theo }}$ was calculated for the kinematics of every electron that passed the required electron cuts. This value was placed in the corresponding $Q^{2}$ bin and finally the mean $A_{\text {elas }}^{\text {theo }}$ for that $Q^{2}$ bin was used in the analysis. Similarly the elastic asymmetry for the data was determined by combining all the runs in a setting and extracting the asymmetry in the $\mathrm{W}$ range of $0.92-0.96 \mathrm{GeV}$. The experimental elastic asymmetry was determined for several $Q^{2}$ bins and divided by the average calculated asymmetry, see Fig 4.14. An average over the chosen $Q^{2}$ bins gave the final value of $P_{b} P_{t}$. Table 4.2 represents the extracted $P_{\text {beam }} P_{\text {target }}$ for each beam and target setting.

Due to the fiducial problems in the $2 \mathrm{GeV}$ outbending setting the elastic peak in the $Q^{2}$ bins below 0.27 was not used in the beam and target polarization product. These low $Q^{2}$ bins correspond to small scattering angles for the electron. Small angle scattering is detected by the edges of the CLAS detector and because of this the elastic peaks are not stable and do not follow the expected $Q^{2}$ dependence. The lack of $Q^{2}$ dependence in the dilution factor and packing fraction suggest that the inelastic data in these low $Q^{2}$ bins are reasonable and may be used in the rest of the analysis. 
Table 4.2: Beam Polarization $\times$ Target Polarization

\begin{tabular}{|c|c|c|c|}
\hline Beam $(\mathrm{GeV})$ & Torus $(\mathrm{A})$ & Target Pol & $P_{B} P_{T}(\%)$ \\
\hline \hline 2.562 & -1500 & plus & $-37.32 \pm 1.30$ \\
\hline 2.562 & -1500 & minus & $39.91 \pm 1.05$ \\
\hline 2.565 & 2250 & plus & $-27.88 \pm 0.69$ \\
\hline 2.565 & 2250 & minus & $36.59 \pm 0.84$ \\
\hline 2.565 & 1500 & plus & $-27.90 \pm 1.52$ \\
\hline 2.565 & 1500 & minus & $34.14 \pm 1.02$ \\
\hline 4.278 & 2250 & plus & $-18.18 \pm 1.67$ \\
\hline 4.278 & 2250 & minus & $25.53 \pm 1.99$ \\
\hline 4.278 & -2250 & plus & $33.28 \pm 1.77$ \\
\hline 4.278 & -2250 & minus & $-33.97 \pm 1.37$ \\
\hline
\end{tabular}




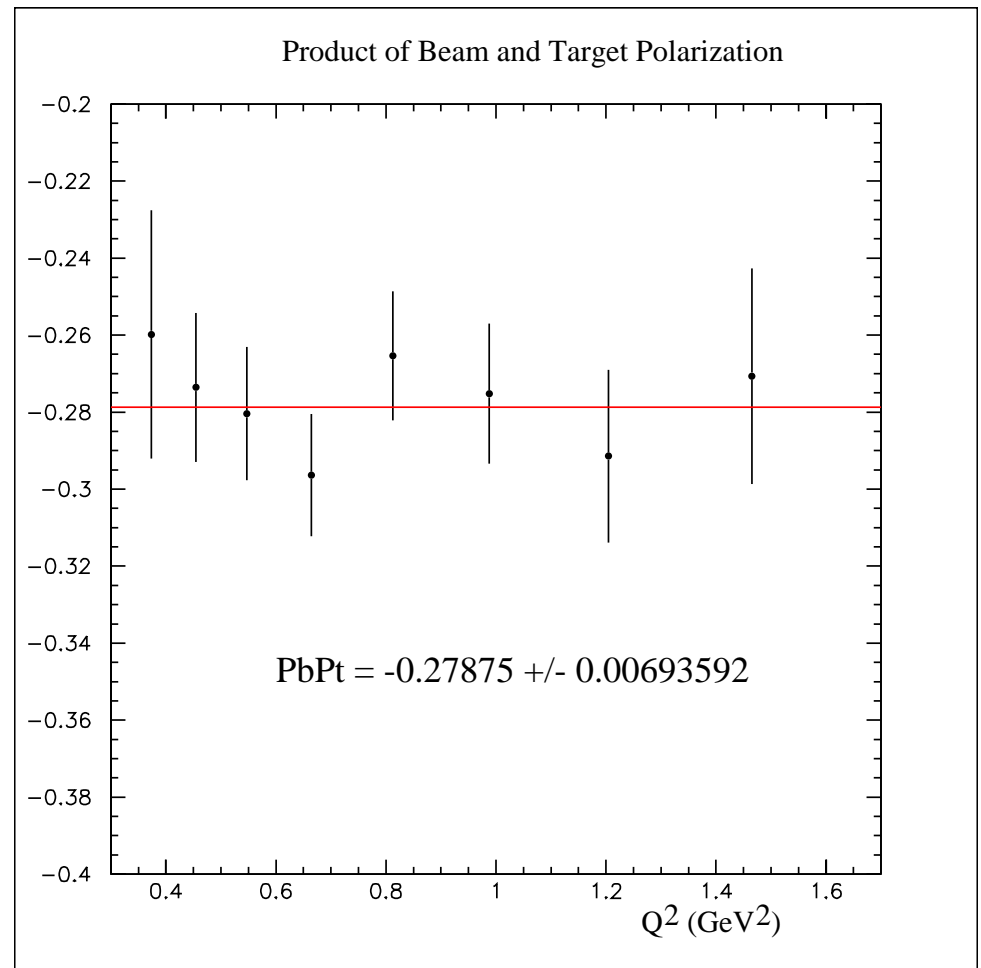

Figure 4.14: The product of the beam and target polarization is extracted by fitting several $Q^{2}$ bins for each beam, target and torus setting. The above plot shows $P_{B} P_{T}$ as a function of $Q^{2}$ for the setting with $2 \mathrm{GeV}$ beam, inbending torus and positive target orientation.

\subsection{Pair Symmetric Correction}

The electrons detected by the CLAS are either scattered from the target or produced in decay reactions. The latter source contaminates the scattered electron sample and must be corrected for. A large portion of these decay electrons have been removed by the vertex cut since their tracks would not have been traced back to the target by the reconstruction code. The primary source of these decay product electrons are neutral pions, which are produced copiously in photoproduction. It is possible then 
that the vertex cut is not sufficient and that a significant correction is required for the high $W$ regions.

There are two neutral pion decays which contribute to the pair symmetric background. The reaction $\pi^{0} \rightarrow e^{+} e^{-} \gamma$ occurs with a $1.2 \%$ decay probability. The dominant channel, $\pi^{0} \rightarrow \gamma \gamma$ produces $e^{+} e^{-}$from pion production. It is conceivable as well that lepton production from bremsstrahlung photons could contribute to the pair symmetric background by $\gamma \rightarrow e^{+} e^{-}$. Calculations using formulas from Tsai[67], show that the probability of decay from these forward angle photons is negligible and therefore this channel was disregarded.

The pair symmetric correction to the inclusive raw asymmetry $\mathrm{A}_{\|}^{\text {raw }}$ is written as:

$$
A_{\|}=A_{\|}^{\text {raw }} \frac{\left(1-R_{r} R_{a}\right)}{1-R_{r}}
$$

where $R_{r}$ is the ratio of $e^{+}$to $e^{-}$rates, and $R_{a}$ is the asymmetry, $A_{\|}^{+} / A_{\|}^{-}$, for $e^{+}$ and $e^{-}$. An analysis note written by Peter Bosted[67] describes the procedure used to find the ratios $R_{a}$ and $R_{r}$ using the EG1 data. Since a positron trigger was not included in the EG1 run the first step was to re-run RECSIS on a small set of runs. Reprocessing permitted tracks from positive particles to be reconstructed. The $e^{+}$ rates for specific $Q^{2}$ and $\mathrm{W}$ bins were then compared to electron rates for runs with the same energy but opposite torus setting, see Fig. 4.15. Opposite torus settings ensured that the rates for both particles reflected the same kinematic and fiducial regions of CLAS. In the same way the asymmetries for both sets of runs were extracted. It is clear that the pair symmetric background contribution increased dramatically for the higher $\mathrm{W}$ bins. These results were compared to a model which calculates the ratio of 
pair symmetric electrons to the total number observed:

$$
R_{a}^{\text {model }}=\frac{\sigma_{\text {pair }}}{\sigma_{\text {pair }}+\sigma_{\text {born }}}
$$

The data reflect radiated cross sections and not Born so it should be expected that the model will over-estimate the $R_{A}$ in the data. This is true for the lower $Q^{2}$ bins but the effect is reversed for higher $Q^{2}$. The reason for this could be due to electron or positron samples which are contaminated by the corresponding pions.
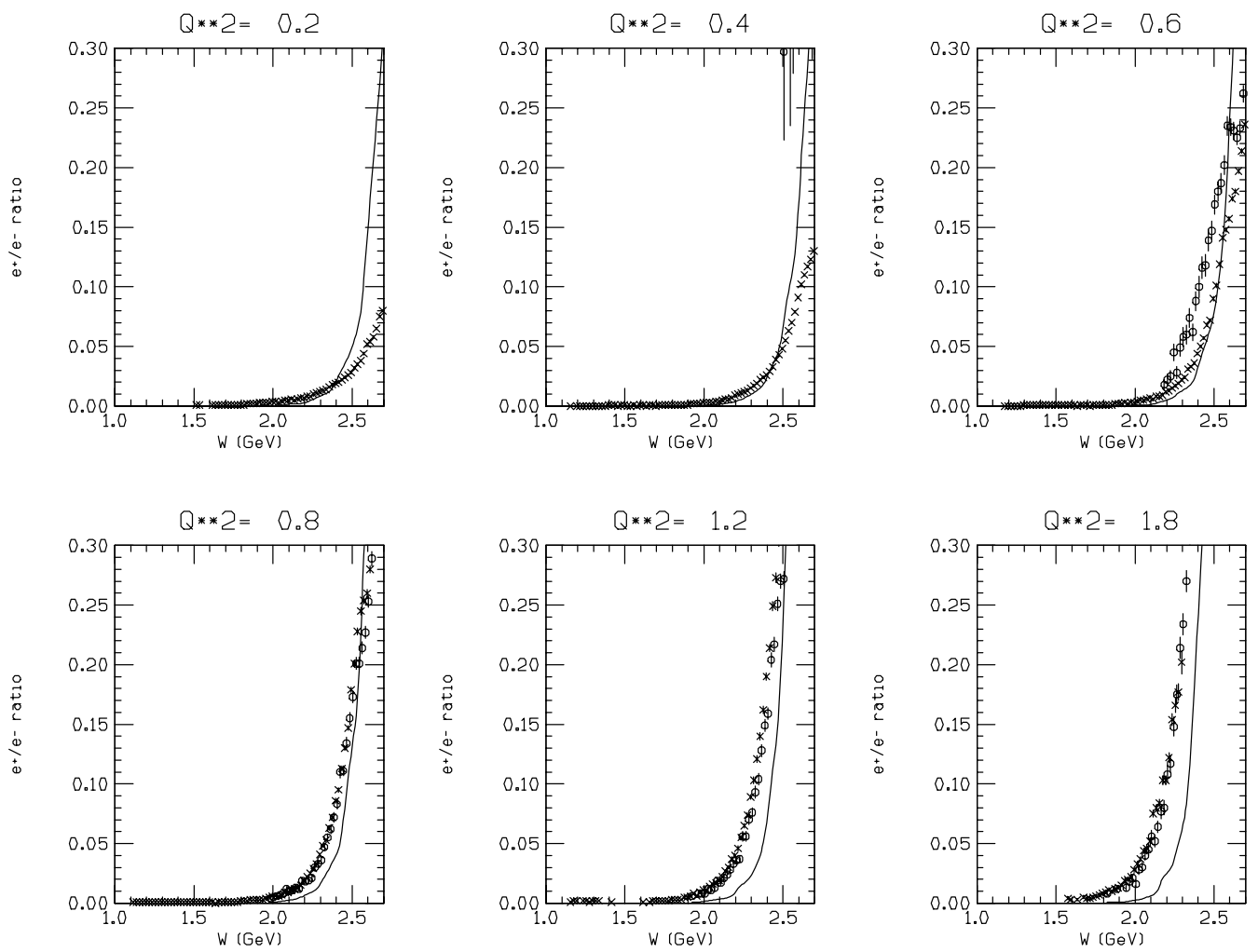

Figure 4.15: The ratio of positrons to electrons for the $4 \mathrm{GeV}$ data. The crosses(circles) represent inbending(outbending) torus field configurations.

The inclusive proton analysis applied the pair-symmetric correction by accessing 
the file eg1_posrat.tbl, which contains $R_{r}$ for the desired beam energy, $Q^{2}$ and $\mathrm{W}$ bin. Studies of $R_{a}$ show the value to be consistent with zero for all beam energies, $-0.3 \pm 0.4$ for $2 \mathrm{GeV}$, and $-0.3 \pm 0.25$ for $4 \mathrm{GeV}$.

\subsection{Radiative Corrections}

The formulas for the asymmetries used in deriving $g_{1}$ are based on the assumption of one photon exchange, which is the Born process to first order in $\alpha$. There are higher order contributions involved, four of which are shown in Figures $4.16-4.19$. The radiative corrections remove the contribution from these higher order diagrams.

There are two types of radiative corrections, internal and external. External corrections account for processes that remove energy from the incoming and outgoing electron. Figures 4.16 and 4.17 show the bremsstrahlung of photons by the electron before and after the interaction, which alters the inital and final energies. Similar diagrams exist for the proton but are disregarded because $M_{p}>>M_{e}$ and therefore the corrections are much smaller. Higher order diagrams which consist of two or more photon emission are also disregarded because they are suppressed by a factor of $\left(1 / \alpha_{e m}\right)^{\text {order }}$. The effect of external radiation is most noticeable in the asymmetric widening of the elastic peak, which is called the elastic radiative tail.

Internal corrections account for higher order processes which occur during the electron-hadron interaction. The vertex correction, Fig. 4.18, accounts for the emission of a photon by the incoming electron and reabsorption of the same photon by the scattered electron. This diagram also exists for the proton but is disregarded. Figure 4.19 accounts for the vacuum polarization of the photon exchanged between 


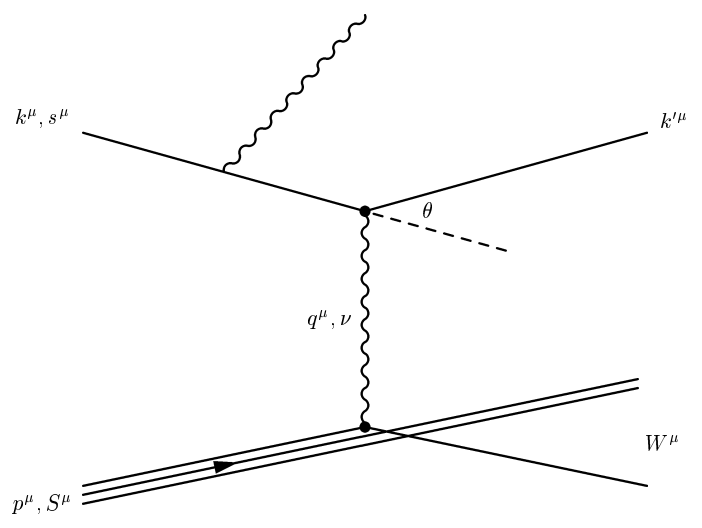

Figure 4.16: Bremsstrahlung radiation emitted from incoming electron

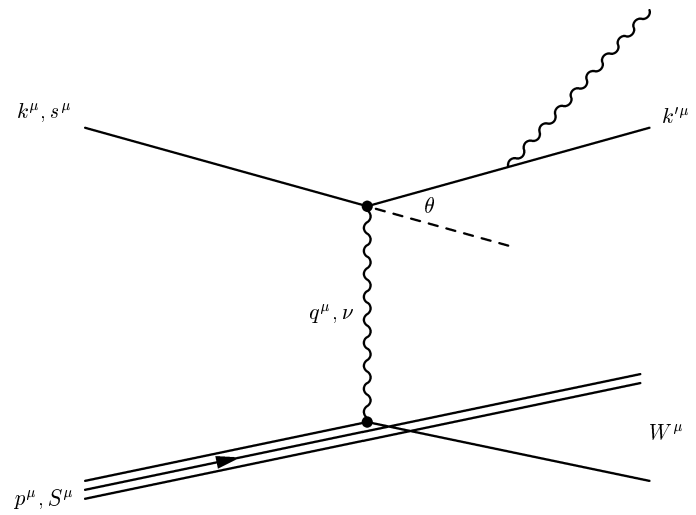

Figure 4.17: Bremsstrahlung radiation emitted from scattered electron

the lepton and hadron. Figure 4.16 and 4.17 also contribute to internal corrections since it is possible for the bremsstrahlung to also occur during the interaction.

The code used for the EG1 inclusive radiative corrections is based on RCSLACPO [69], which utilizes methods developed by Shumeiko and Kuchto[73] for the internal corrections, and Tsai[74] for the external corrections. The code also uses the polarized and unpolarized cross-section models to determine the Born asymmetry. These models are the same ones as used in the "Models" program and extend the kinematic 


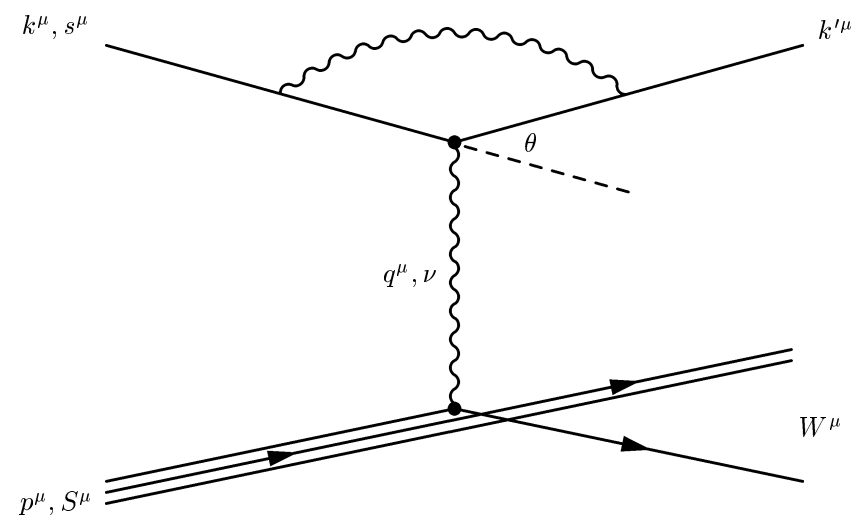

Figure 4.18: Vertex Correction

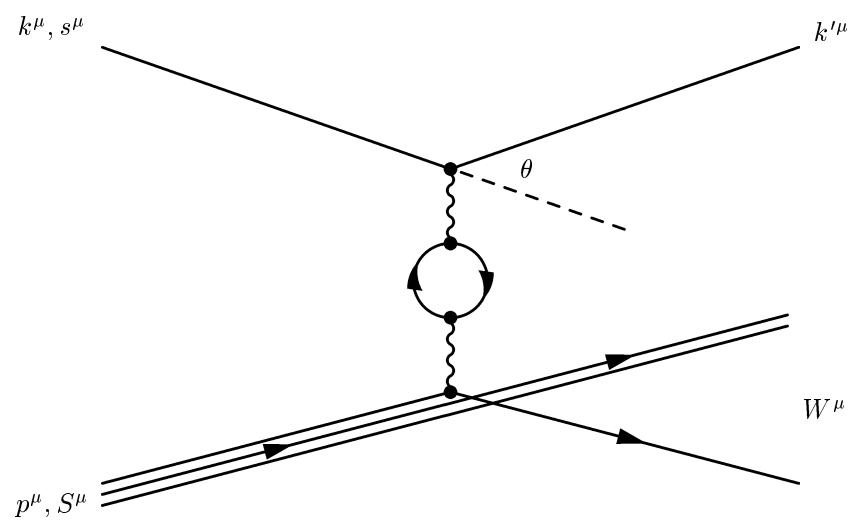

Figure 4.19: Vacuum Polarization

range of RCSLACPOL down to the resonance region.

It is necessary to calculate both the Born and radiated, polarized and unpolarized cross-sections in order to derive the radiative corrections needed for asymmetries.

$$
A_{\|}=\frac{\sigma^{\uparrow \downarrow}-\sigma^{\uparrow \uparrow}}{\sigma^{\uparrow \downarrow}+\sigma^{\uparrow \uparrow}}=\frac{\sigma^{\text {pol }}}{\sigma^{\text {unpol }}}
$$

The calculation of the externally radiated cross-sections, for both the polarized 
and unpolarized case, is composed of an elastic, quasi-elastic and inelastic piece.

$$
\sigma_{\text {total }}=\sigma_{\text {born }}(1+\delta)+\sigma_{\text {elas }}+\sigma_{\text {quasi }}+\sigma_{\text {inelas }}
$$

The internal corrections are calculated in the Born approximation and take into account the electron vertex corrections, $\delta_{\text {vert }}$, and the leptonic and hadronic vacuum polarization corrections, $\delta_{v a c}^{l}$ and $\delta_{v a c}^{h}$.

$$
\delta=\delta_{v e r t}+\delta_{v a c}^{l}+\delta_{v a c}^{h}
$$

The correction applied to the EG1 data is $A_{\text {diff }}$, which is the difference between the Born and the radiated asymmetry produced by the code.

$$
A_{\text {diff }}=A_{\text {Born }}-A_{\text {rad }}
$$

Raw asymmetries are corrected by adding $A_{\text {diff }}$,

$$
A_{\|}^{\text {corr }}=A_{\|}+A_{\text {diff }}
$$

\subsection{Live-time Correction}

Hardware limitations require that the detector stop registering data while the information from an event is being collected by the Data Aquisition System (DAQ). This results in a dead-time which was $\sim 10 \%$ on average during the EG1 run. Systematic errors may occur in an asymmetry analysis if the dead-time, and thus the live-time, 
is helicity dependent. Ideally the helicity dependent live-time could be calculated directly from scalers which contain gated and ungated helicity information. Unfortunately there were no gated scalers with helicity information recorded in the EG1 run. Instead the live-time is deduced by taking the ratio of the gated clock to the ungated Faraday cup. This live-time varies with the trigger rate in a linear way:

$$
\text { live }- \text { time }=A+\left(B \times N_{T}\right)
$$

$N_{T}$ is the number of triggers in a 1 second interval and the constants $\mathrm{A}$ and $\mathrm{B}$ are fit parameters which are characteristic of the particular data set. The live-time correction is applied by multiplying the ungated Faraday cup values in the DST by live-time for that beam and torus setting. This procedure in essence recreates the gated helicity scaler. The sum of the live-time corrected Faraday cup readings for each helicity are then used in the beam charge asymmetry normalization. Table 4.3 contains the average live-time correction as well as the constants A and B for each beam and torus setting.

\subsection{Polarized ${ }^{15} \mathrm{~N}$ Correction}

Polarized background nuclei in the target can dilute or enhance the proton asymmetry and therefore must be corrected for. The ${ }^{15} \mathrm{~N}$ in the ammonia as well as the $2 \%$ contamination of ${ }^{14} N$ were both polarizable. In this analysis the ${ }^{14} N$ contamination was considered negligible and no correction was applied. Target data taken during SLAC experiment E143[77] mapped out the ${ }^{15} N$ polarization as a function of the 


\begin{tabular}{|c|c|c|c|c|c|}
\hline Beam $(\mathrm{GeV})$ & Torus $(\mathrm{A})$ & Target Pol & Live-time $(\%)$ & $\mathrm{A}$ & $\mathrm{B}$ \\
\hline \hline 2.562 & -1500 & plus & 0.003 & 1.0004 & $-2.78 \times 10^{-5}$ \\
\hline 2.562 & -1500 & minus & 0.036 & 1.0004 & $-2.78 \times 10^{-5}$ \\
\hline 2.565 & 2250 & plus & 0.032 & 1.0055 & $-3.75 \times 10^{-5}$ \\
\hline 2.565 & 2250 & minus & 0.002 & 1.0055 & $-3.75 \times 10^{-5}$ \\
\hline 2.565 & 1500 & plus & 0.032 & 1.006 & $-3.61 \times 10^{-5}$ \\
\hline 2.565 & 1500 & minus & 0.002 & 1.006 & $-3.61 \times 10^{-5}$ \\
\hline 4.278 & 2250 & plus & 0.019 & 1.011 & $-4.23 \times 10^{-5}$ \\
\hline 4.278 & 2250 & minus & 0.048 & 1.011 & $-4.23 \times 10^{-5}$ \\
\hline 4.278 & -2250 & plus & 0.007 & 1.0063 & $-3.41 \times 10^{-5}$ \\
\hline 4.278 & -2250 & minus & 0.044 & 1.0063 & $-3.41 \times 10^{-5}$ \\
\hline
\end{tabular}

Table 4.3: The live-time correction in percent for each beam, torus and target setting.

$\mathrm{NH}_{3}$ polarization. The unpaired proton in the ${ }^{15} \mathrm{~N}$ contributes to the measured proton asymmetry proportionally to the nitrogen polarization and with a negative sign because of the negative magnetic moment of ${ }^{15} \mathrm{~N}$. The following empirical relation was used to determine the ${ }^{15} \mathrm{~N}$ polarization during EG1:

$$
P_{15 N}=0.136\left|P_{p}\right|-0.183\left|P_{p}\right|^{2}+0.335\left|P_{p}\right|^{3}
$$

The ${ }^{15} N$ polarization is used in the multiplicative correction, $C_{1}$, to the raw asymmetry:

$$
C_{1}=1-\frac{1}{3} \frac{1}{3} \frac{P_{15} N}{\left|P_{P}\right|} g_{E M C}(x)
$$

Here $g_{E M C}(x)$ is the correction for the European Muon Collaboration(EMC) effect[79] taken at atomic mass number 15. Clebsh-Gorden coefficients for the nitrogen 
wave function require the first factor of $-\frac{1}{3}$. The second factor of $\frac{1}{3}$ reflects the ratio of hydrogen to nitrogen atoms in the target material. The ${ }^{15} N$ polarization correction is applied by multiplying the raw proton asymmetry by $C_{1}$, with the effect of reducing the asymmetry by $1-2 \%$.

\subsection{Systematic Errors}

Systematic errors are the result of two types of uncertainties. The first is the uncertainty associated with models used to analyse the data, such as the parameterizations of the structure functions $A_{2}, F_{1}$ and R by "Models". The second type of systematic error arises from uncertainties in the experimental apparatus, for example the actual distance between the banjo entrance and exit windows during the run. The following sections will discuss the types of systematic errors that were considered and applied to the results of this analysis. Table 4.4 lists these systematic tests and the notation used to represent them in the text. A graphical representation of these errors is shown for $g_{1}, \Gamma_{1}$ and the Generalized GDH sum rule in Figs. 4.20 - 4.22.

The general procedure for the systematic error analysis involves changing a model or parameter to an alternate value, which is reasonable but not believed to be as valid as the standard value, and then recalculating the results $A_{\|}, A_{1}+\eta A_{2}, g_{1}\left(W, Q^{2}\right)$, $\Gamma_{1}^{P}$ and the generalized GDH integral. The difference between the altered result and the "standard" one is then used as the systematic error. The appropriate systematic errors are added in quadrature and displayed as a systematic error bar. For the results involving $g_{1}$ the systematic error is added in quadrature with the statistical error. 


\begin{tabular}{|c|c|}
\hline Systematic Error Parameter & Description \\
\hline \hline $\mathrm{L}$ & vary L by $10 \%$ \\
\hline$F_{1}^{N} / F_{1}^{P}$ & vary $F_{1}^{N} / F_{1}^{P}$ by $10 \%$ \\
\hline $\mathrm{PS}$ & remove pair symmetric correction \\
\hline $\mathrm{F} 1$ & ${\text { Bodek parameterization for } F_{1}}^{A_{2} \text { systematic as detailed in section } 4.17 .4}$ \\
\hline $\mathrm{A} 2$ & $\mathrm{R}$ systematic as detailed in section 4.17 .4 \\
\hline $\mathrm{R}$ & vary $\mathrm{W}$ region used for $P_{B} P_{T}$ \\
\hline elastic & Remove live-time calculation \\
\hline $\mathrm{LT}$ & Remove electroweak calculation \\
\hline $\mathrm{EW}$ & Remove ${ }^{15} N$ correction \\
\hline $\mathrm{N} 15$ &
\end{tabular}

Table 4.4: Systematic Error Summary and Notation

\subsubsection{Background Subtraction}

The systematic error in the simulation of the background $\left(15^{N}\right.$, helium and windows) comes from two parameters. The first is the unknown distance between the banjo entrance and exit windows, L, during normal operating procedures. The standard value for $\mathrm{L}$ is $1.5 \mathrm{~cm}$ and it was varied by $10 \%$ in order to estimate the systematic error. The second parameter is the ratio of $F_{1}^{N} / F_{1}^{P}$ in the inelastic region. This ratio was varied by $10 \%$ and again the difference from the standard used as the systematic. Several models for the elastic form factors $G_{E N}, G_{E P}, G_{M N}$ and $G_{M P}$ were used to look at the systematic in the elastic region. The variation of these models made absolutely no difference in the results, which was expected because the elastic form factors are known very well for the kinematic region of interest. 


\subsubsection{Beam $\times$ Target Polarization}

The systematic associated with the extraction of the product of the beam and target polarization is heavily correlated with the systematic associated with the background subtraction. The only additional systematic was the $\mathrm{W}$ region used to find the average elastic asymmetry. In the standard analysis all settings, except the $2 \mathrm{GeV}$ outbending data, the elastic asymmetry was averaged over $\mathrm{W}=0.92-0.96 \mathrm{GeV}$. This was widened to $0.9-0.98$ for systematic studies. For the $2 \mathrm{GeV}$ outbending data the standard range is $0.9-0.98$ and this was reduced to $0.92-0.96$ for systematic studies.

\subsubsection{Pair Symmetric, Live-time and ${ }^{15} N$ Correction}

The pair symmetric correction is small and therefore the systematic error was estimated by completely eliminating the correction. The same procedure was used for the live-time correction.

\subsubsection{Models}

The largest source of systematic uncertainty is due to the parameterizations of the structure functions from "Models". All the results make use of the function, R. The systematic tests for R included using the parameterization by Ricco [76], as well as setting $\mathrm{R}$ to a constant value of 0.25 . The systematic for the unpolarized structure func-

tion, $F_{1}$, was found by using the parameterization given by Bodek [75]. The function $A_{2}$ has the least amount of data input and is highly model dependent. The standard output used for $A_{2}$ is the fit from "Models" which incorporates the MAID2000[41] model. The systematic was estimated by using the output from "Models" which does 
not incorporate the MAID2000 output. This model produces a more negative function in the region from the $\triangle$ to pion threshold. Another systematic study set $A_{2}$ to zero for the resonance region up to $\mathrm{W}=2 \mathrm{GeV}$.

Systematic errors for the deep inelastic contribution of $g_{1}$ to the first moment were estimated in three ways. The first method uses extrapolations for $g_{1}$ from the parameterization by Simula [34]. In addition two variations on the "Models" standard parameterization were used. The standard output contains a fit to all deep inelastic scattering world data on $A_{1}$, including EG1 data (for W $2 \mathrm{GeV}$ ) and the most recent low- $Q^{2}$ and high-x Heremes [29] data. The first variation, "Models0", is the published parameterization [80] of world data for $A_{1}$ with minimal low $Q^{2}$ and high-x constraints. "Models0" only contains deep inelastic scattering data published before 1999. The second variation, "Models2", includes all deep inelastic data published before 2000 and improved behavior of $A_{1}$ in the low $Q^{2}$, high x region.

Systematic error for the deep inelastic contribution to the Generalized GDH integral without the longitudinal-transverse component was calculated using three different models for the term $A_{1} F_{1}$. The first two variations used the standard "Models" output for $F_{1}$ while using "Models0" and "Models2" output for $A_{1}$. The third model used the standard $A_{1}$ "Models" output but instead used the Bodek fit for $F_{1}$.

\subsubsection{Radiative Correction}

The radiative correction systematic was calculated by varying the models used in the code. The following is a list of the variations:

A. Vary elastic model

B. Set $\mathrm{A}_{\perp}=0$ 
C. Set $\mathrm{A}_{2}=0$

D. Set $g_{2}=0$

D. Use Ricco R instead of R from "Models"

E. Use "Models0" for $\mathrm{A}_{1}$

F. Use "Models2" for $\mathrm{A}_{1}$

G. Use standard "Models" output without EG1 data for W>2 GeV

The root mean square differences between these variations and the standard output from "Models" define the systematic error. The average error on the correction was found to be $\sim 10 \%$. The radiative correction contribution to the systematic error is not included in the plots shown in Chapter 5 .

\subsubsection{Electroweak Interaction}

The parity violating electroweak interaction involving exchange of $\mathrm{W}$ and $\mathrm{Z}$ bosons can result in a beam polarization dependent asymmetry so that:

$$
\sigma^{\uparrow \Uparrow} \neq \sigma^{\downarrow \Downarrow}
$$

This effect can be removed by taking exactly the same amount of data for each target polarization, assuming the magnitude of each polarization direction were equal. The probability an electroweak interaction occurring is greatly reduced at small $Q^{2}$ so no attempt was made to measure it in EG1. The derivation of the correction in the literature[81] applies to isoscalar targets and is applicable to the proton target 
used in EG1. The electroweak correction is estimated to be [82]:

$$
C_{E W}=10^{-4} \times Q^{2} \times P_{\text {beam }}
$$

This correction is $\sim 3.5 \times 10^{-5}$, orders of magnitude smaller than the statistical error of the raw asymmetries calculated in this analysis. For this reason the electroweak correction is not included in this analysis. Instead the correction value, $C_{E W}$, was added to all raw asymmetries, and the deviation from the standard calculation was used as the an indication of the systematic error due to the electroweak interaction. 


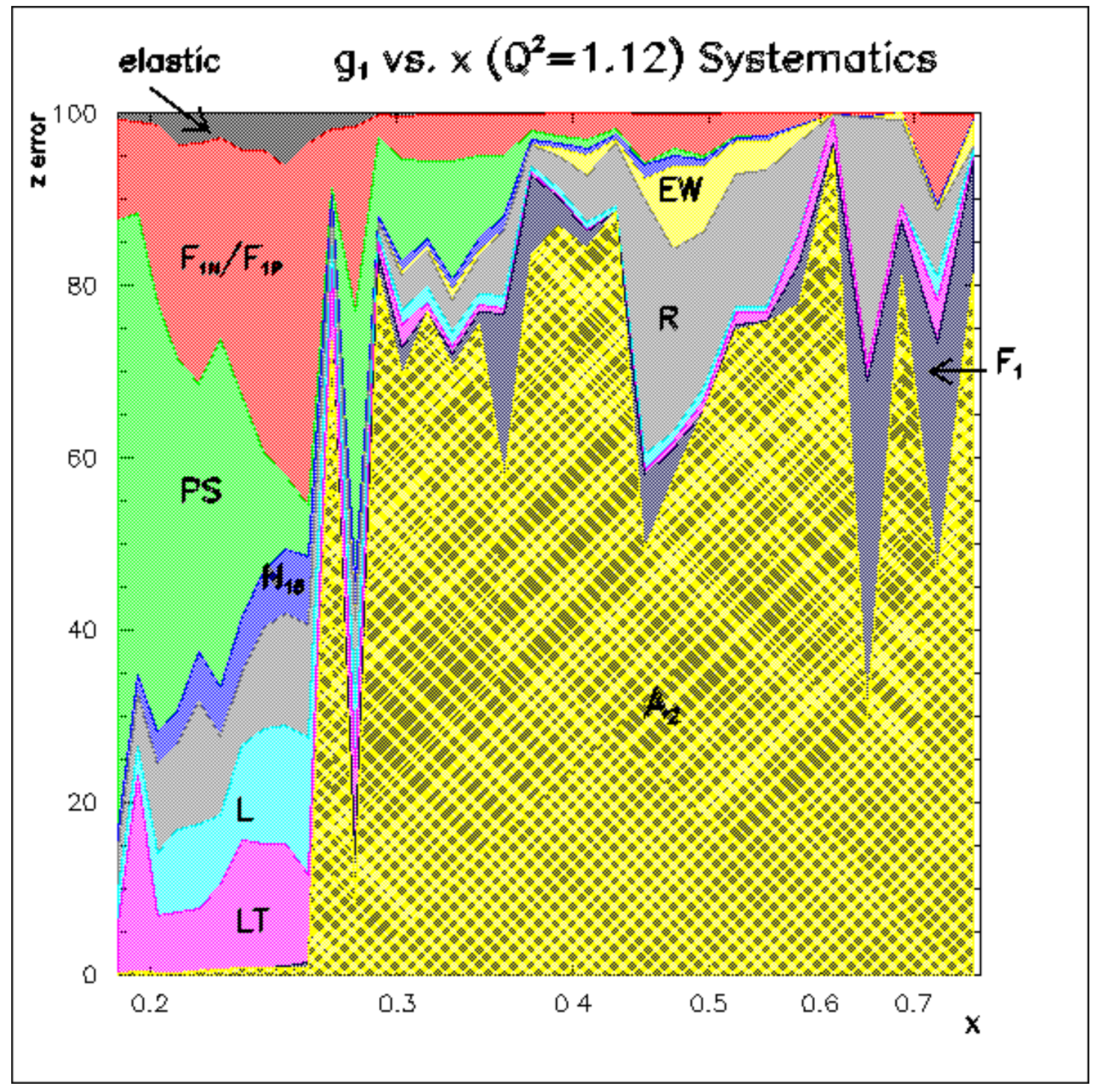

Figure 4.20: 


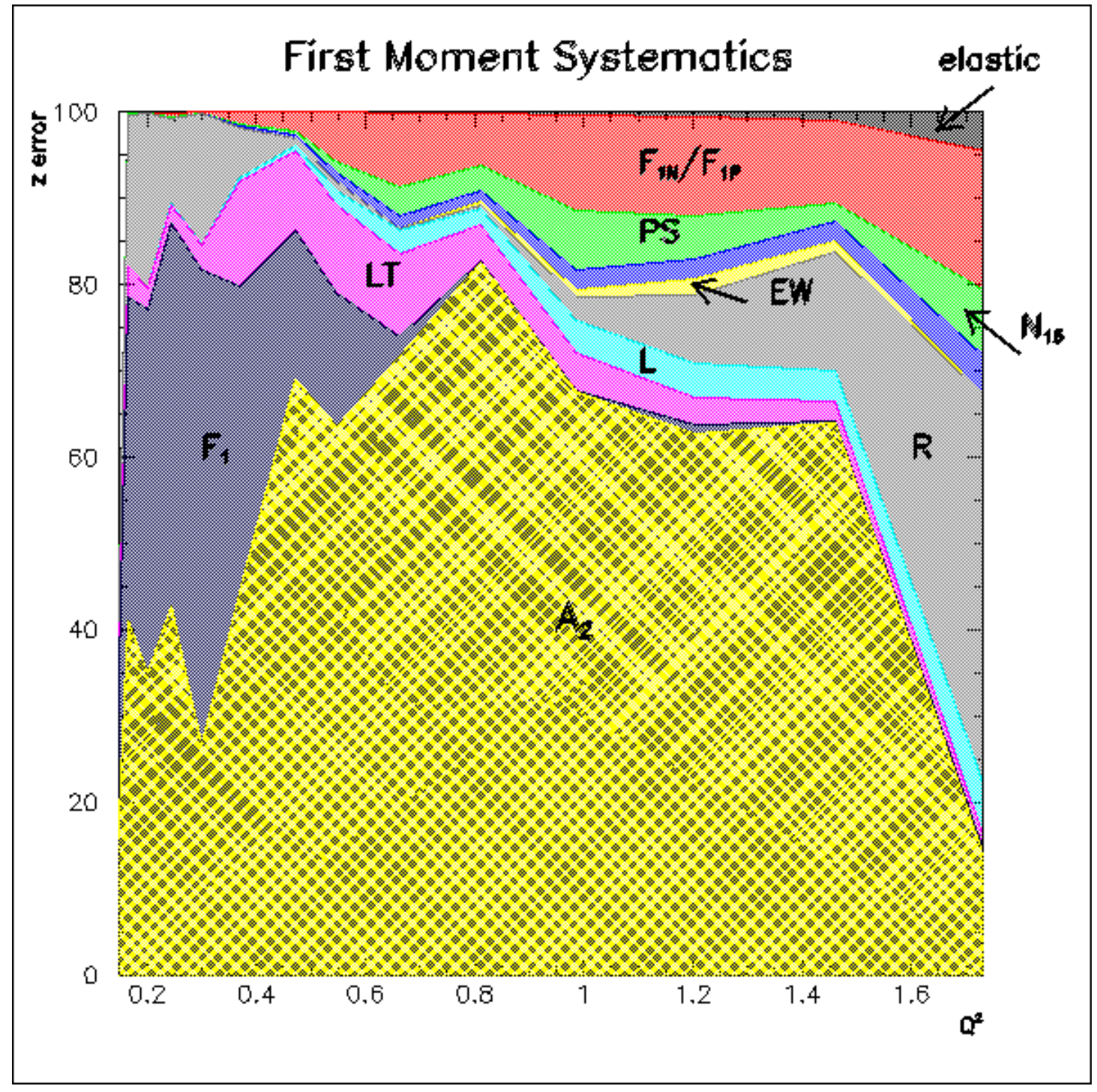

Figure 4.21: 


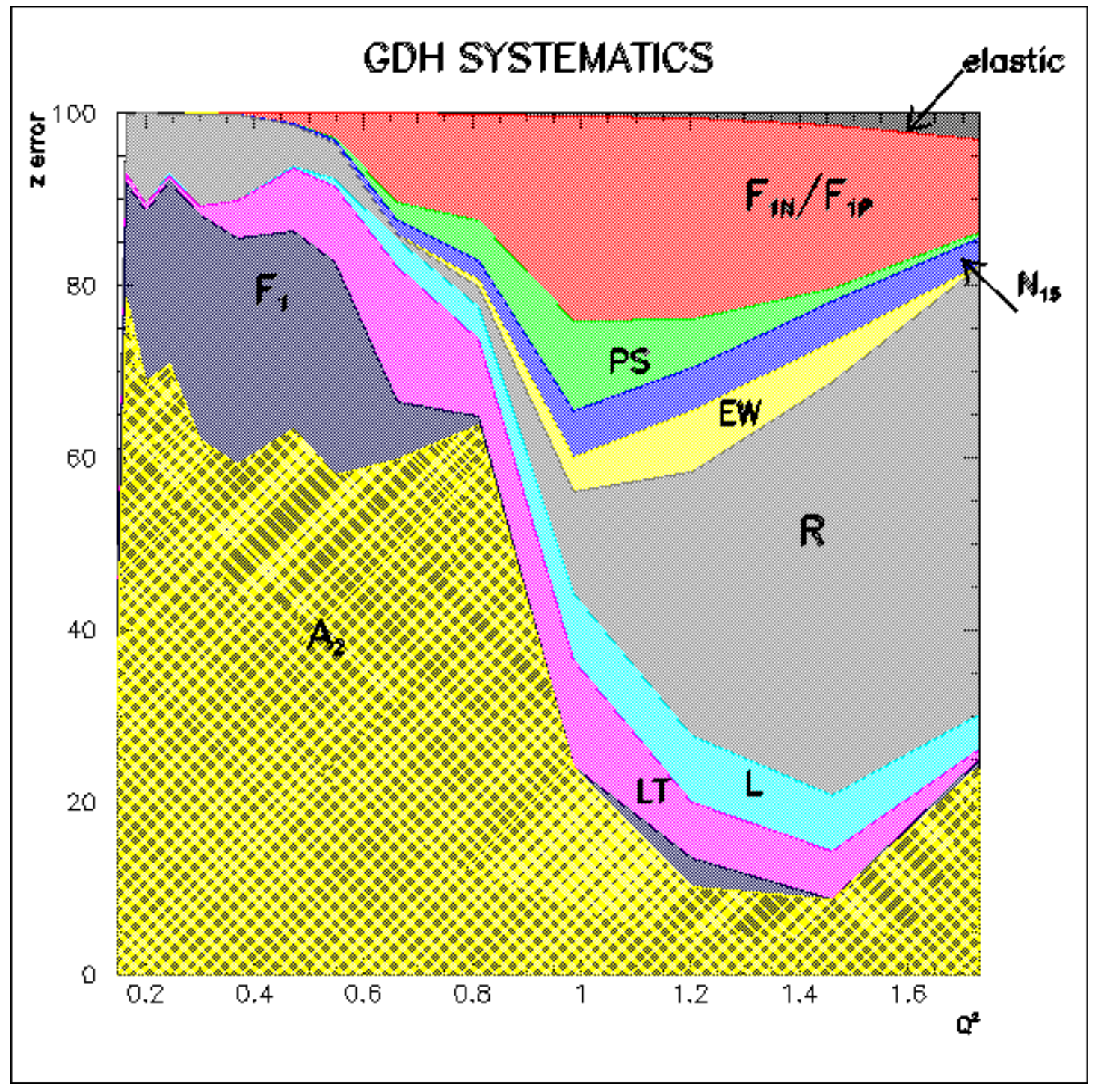

Figure 4.22: 


\section{Chapter 5}

\section{Results}

Table 5.1 lists the number of events obtained on the proton target for each setting of the beam, torus current and target polarization. The fully corrected $A_{\|}$was calculated for each of these ten settings. From these measurements, along with input from "Models", quantities such as $A_{1}+\eta A_{2}, g_{1}^{p}$ and the first moment $\Gamma_{1}^{P}$ can be obtained. Throughout the analysis it was necessary to use the parameterizations computed by "Models" to calculate the quantities, $A_{2}, F_{1}$, and $\mathrm{R}$ in the resonance region as well as $g_{1}$ in the region of $\mathrm{W} \geq 2 \mathrm{GeV}$. A description of the analysis, including a detailed accounting of where the model was used, and results will be shown in the following sections.

\section{$\mathbf{5 . 1} A_{1}+\eta A_{2}$}

The asymmetry $A_{1}+\eta A_{2}$ is relatively simple to extract from the measured $A_{\|}$.

$$
A_{1}+\eta A_{2}=A_{\|} / \mathcal{D}
$$




\begin{tabular}{|c|c|c|c|c|c|c|}
\hline Setting & Pol. & \# trig & ${\text { \# DST } \mathrm{e}^{-}}$ & ${\text {\# good } \mathrm{e}^{-}}$ & $\% e^{-} /$trig & $\% e^{-} / \mathrm{DST}$ \\
\hline \hline $2.562-1500$ & + & 120.95 & 89.90 & 59.74 & 49.39 & 66.45 \\
\hline $2.562-1500$ & - & 188.13 & 113.73 & 77.03 & 40.95 & 67.73 \\
\hline 2.5652250 & + & 143.00 & 58.33 & 34.37 & 24.03 & 58.92 \\
\hline 2.5652250 & - & 82.31 & 43.19 & 25.44 & 30.91 & 58.90 \\
\hline 2.5651500 & + & 106.64 & 19.04 & 11.13 & 10.43 & 58.46 \\
\hline 2.5651500 & - & 86.55 & 46.26 & 26.91 & 31.09 & 58.13 \\
\hline 4.2782250 & + & 256.86 & 60.99 & 24.41 & 9.50 & 40.02 \\
\hline 4.2782250 & - & 165.15 & 43.87 & 17.48 & 10.58 & 39.84 \\
\hline $4.278-2250$ & + & 280.30 & 72.6 & 42.94 & 15.32 & 59.15 \\
\hline $4.278-2250$ & - & 127.87 & 118.08 & 69.99 & 54.74 & 59.27 \\
\hline
\end{tabular}

Table 5.1: This tables outlines the number of triggers taken for each beam and torus setting, as well as the percentage of these triggers stored in the DSTs and eventually used in the final data sample. All units are $10^{6}$ electrons. 
The depolarization factor $\mathcal{D}$ accounts for the fact that the virtual photon is not aligned with the direction of the nucleon spin. Using the parameter $\mathrm{R}$ as determined in "Models":

$$
\mathcal{D}=\frac{1-\epsilon \frac{E^{\prime}}{E}}{1+\epsilon R}
$$

$A_{1}+\eta A_{2}$ is dependent on beam energy so that only settings with the same beam energy can be combined. Figure 5.1 shows $A_{1}+\eta A_{2}$ for the $2.56 \mathrm{GeV}$ beam settings. These graphs demonstrate the resolution of the CLAS detector as well the expected $Q^{2}$ evolution of these asymmetries. At small momentum transfer the lower lying resonances, such as the $\triangle(1232)$ are more excited and at larger $Q^{2}$ the transition probability for the higher resonances is greater, resulting in a larger magnitude.

\section{$5.2 g_{1}^{p}$}

The separation of $A_{1}$ and $A_{2}$ is the next step in the extraction of $g_{1}$. Experiment 91-023 was designed to allow a kinematic separation of these two asymmetries by taking advantage of the dependence of $\mathcal{D}$ and $\eta$ on beam energy and $Q^{2}$. The results presented here are based solely on the data from the first run of 91-023 (EG1), which represents about $\sim 1 / 8$ of the total data set. Limited statistics precludes a meaningful kinematic separation and so instead "Models" was used once again to calculate the small contribution of $\eta A_{2}$ to $A_{\|}$, see Fig 5.2.

After subtracting $\eta A_{2}$ it is possible to use both the resulting $A_{1}$ and the calculated $A_{2}$ to construct $g_{1}$.

$$
g_{1}=\frac{\tau}{1+\tau}\left(A_{1}+\frac{1}{\sqrt{\tau}} A_{2}\right) F_{1}
$$




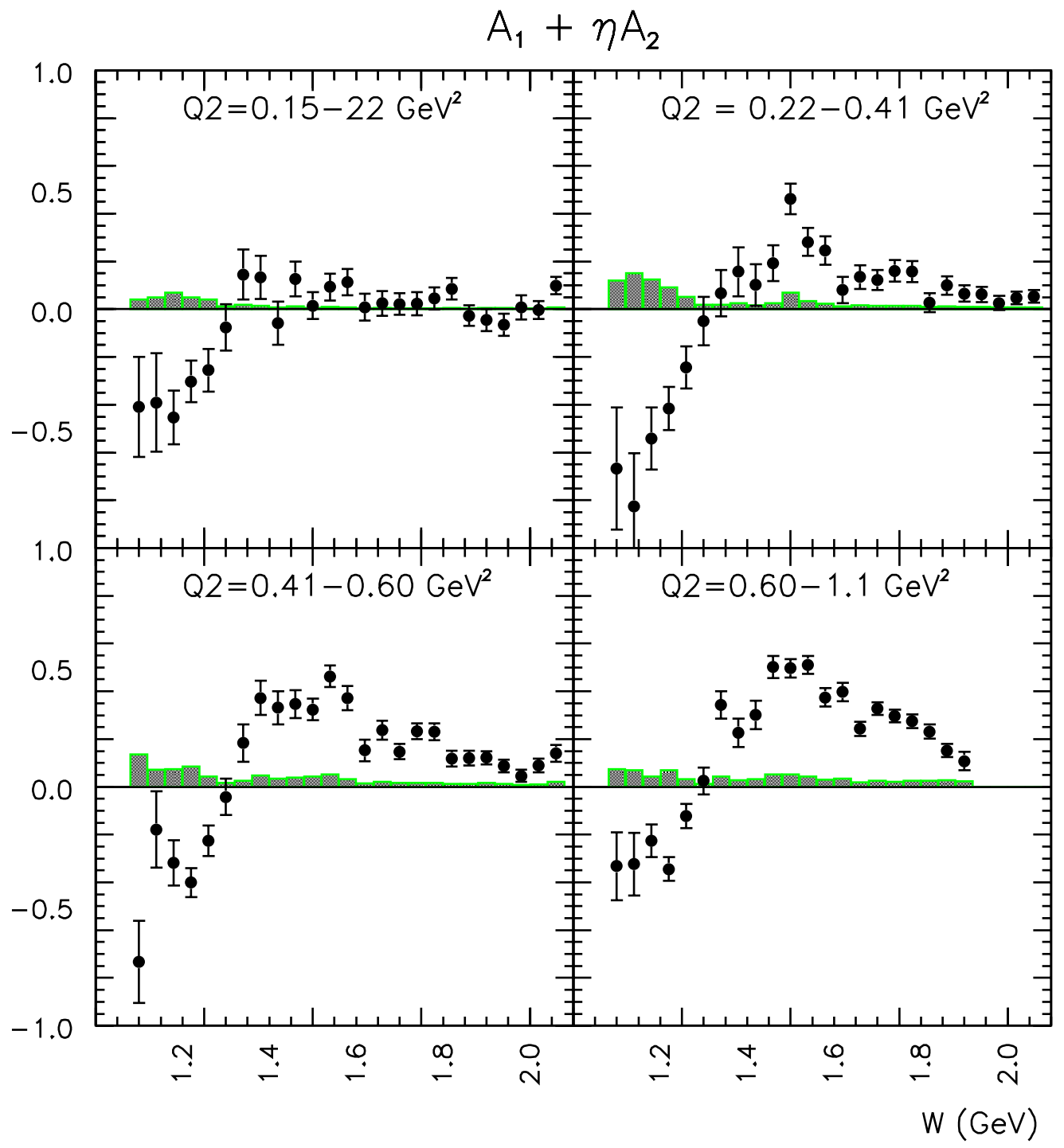

Figure 5.1: $A_{1}+\eta A_{2}$ as a function of $\mathrm{W}$ and $Q^{2}$ for the $2.56 \mathrm{GeV}$ data 


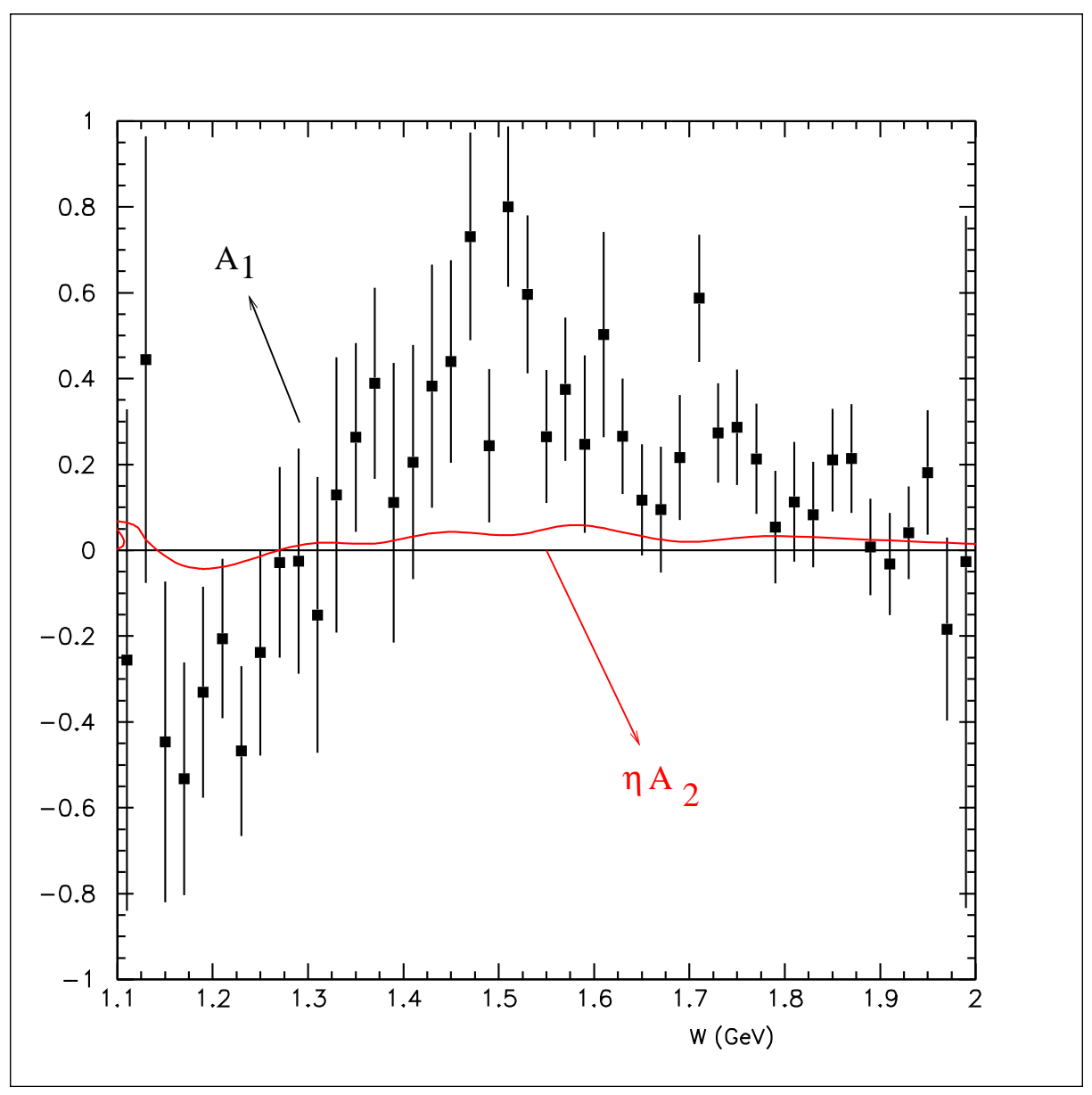

Figure 5.2: Comparison of the term $\eta A_{2}$ (red) to $A_{1}$ (black) for the $Q^{2}$ range of 0.5-0.6 $\mathrm{GeV}^{2}$. 
where $\tau=\nu^{2} / Q^{2}$. In this analysis $g_{1}$ was calculated as a function of $Q^{2}$ and $\mathrm{W}$ for all 5 data settings and then combined using statistical weighting. Figure 5.3 demonstrates the $Q^{2}$ evolution of $g_{1}\left(x, Q^{2}\right)$ for the complete EG1 data set. The lowest $Q^{2}$ shows a significant negative contribution to $g_{1}$ in the $\triangle$ region. As expected this contribution becomes smaller as $Q^{2}$ increases. At $Q^{2}=1.83$ the structure of the resonances are suppressed and $g_{1}$ starts to approach the smooth behavior expected in the scaling region.

Figure 5.4 compares the EG1 data at $Q^{2}=1.12 \mathrm{GeV}^{2}$ to the phenomological models described in Section 2.7.1. The Simula[34] and "Models"[70] curves are in close agreement with the data. This is to be expected, as these models reflect the resonance contributions as well as incorporate the latest experimental data.

\section{$\mathbf{5 . 3} \quad \Gamma_{1}^{P}$}

The contribution of the EG1 data to the first moment, $\Gamma_{1}^{P}$, can be determined from

$$
\Gamma_{1}^{P}=\int_{x} g_{1}\left(x, Q^{2}\right) d x=-\int_{W} g_{1}\left(W, Q^{2}\right) d W *\left(\frac{d x}{d W}\right)
$$

The Jacobian, $J_{n}$, is defined as

$$
J_{n}=\frac{d x}{d W}=-\frac{2 W x}{W^{2}+Q^{2}-M_{p}^{2}}
$$

This integral was first evaluated only for the resonance region, from the pion threshold ( $\mathrm{W}=1.08)$ to $\mathrm{W}=2 \mathrm{GeV}$. This limited integral is of particular interest because there are models which predict the contribution of the resonance region to $\Gamma_{1}^{P}$. 


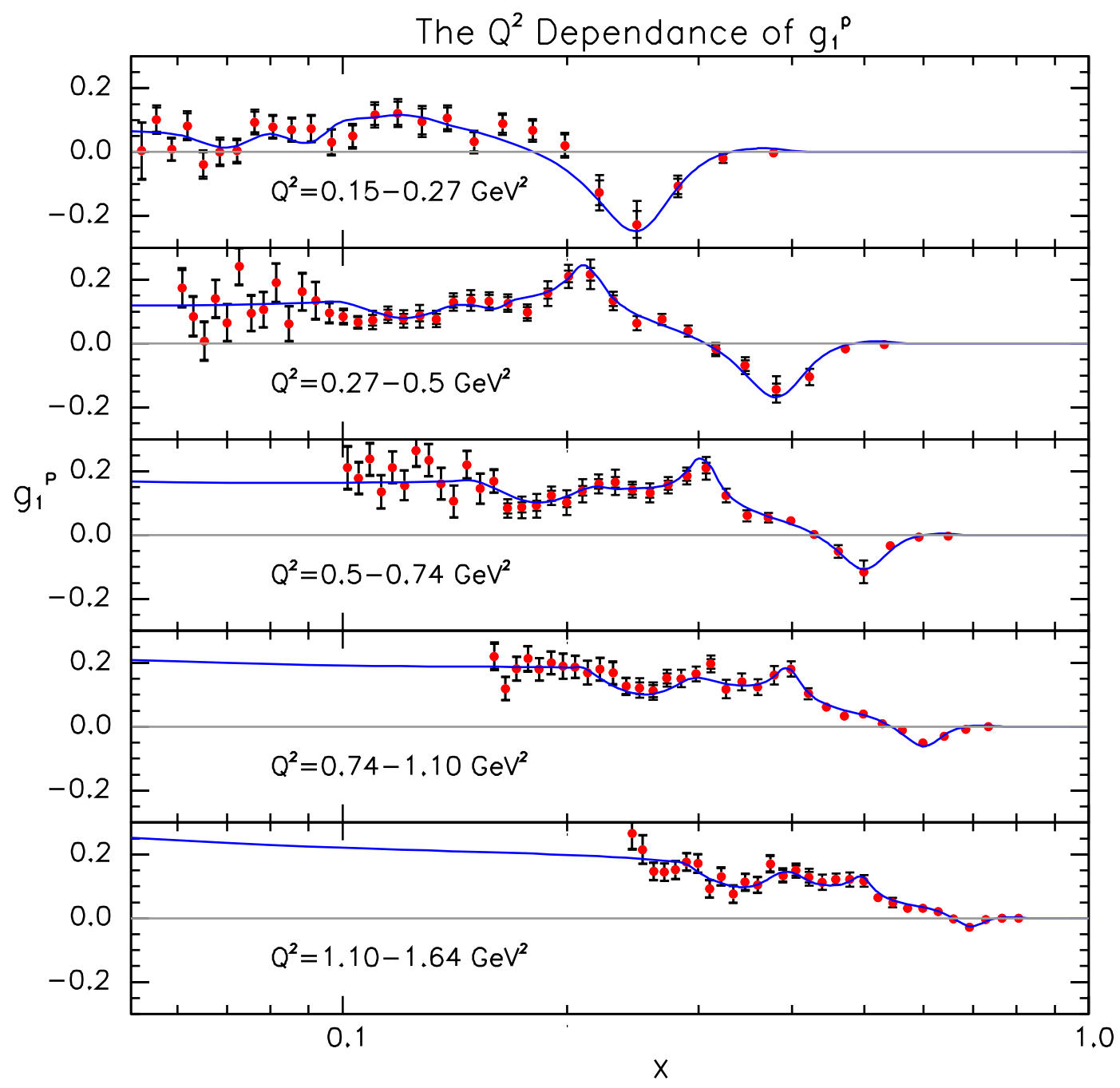

Figure 5.3: The above plots show the $Q^{2}$ evolution of $g_{1}^{p}$ for the EG1 data. The blue curve is the parameterization from "Models". 


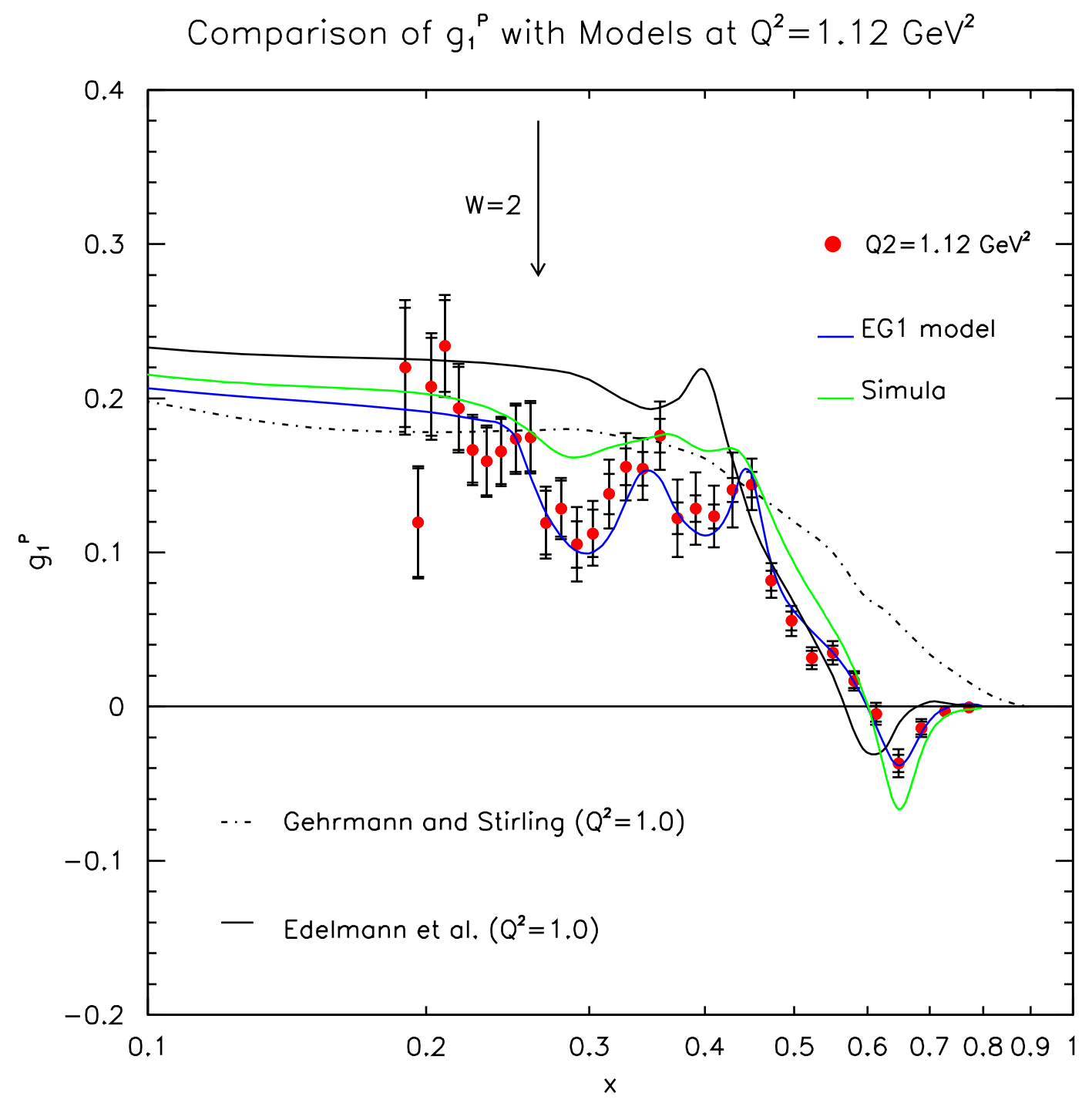

Figure 5.4: The above plot compares the EG1 data for $Q^{2}=1.12 \mathrm{GeV}^{2}$ with models from Simula[34], Gehrmann and Stirling[31] and Edelmann[32]. The blue curve is the $g_{1}^{p}$ parameterization from "Models". The difference between the blue and the green curve is used to calculate the systematic error for the estimation of the deep inelastic contribution to $\Gamma_{1}^{P}$. 
The proton data and models from AO [40] and MAID2000 [41] are shown in Fig. 5.5.

The complete integral for $\Gamma_{1}^{P}$ was obtained by first integrating $g_{1}$ up to the maximum $W$ value, $W_{\text {max }}$, defined by the kinematic phase space of the data. The values for $W_{\max }$ vary as a function of $Q^{2}$ and are shown in Table 5.2. The remaining part of the integral, including the deep inelastic contribution, is determined by using "Models" to predict values of $g_{1}$ down to an $\mathrm{x}=10^{-5}$. These values are integrated and added onto the EG1 data values. The total integral, without the elastic peak, is shown in Fig 5.6. Shown along with the data are models by Ji and Osbourne[37], Burkert and Ioffe[36] and Soffer and Teryayev[39] which are discussed in Section 2.7.2. The slope at $Q^{2}=0$ predicted by the GDH sum rule is shown, as is the prediction of perturbative QCD evolved down to $Q^{2}=1 \mathrm{GeV}[38]$. A fit to the full first moment places the zero crossing at $Q^{2}=0.24 \mathrm{GeV}$, a value which falls between the Soffer/Teryayev and Burkert/Ioffe predictions.

\subsection{The Gerasimov, Drell and Hearn Sum Rule}

The Gerasimov-Drell-Hearn Sum Rule can be tested by using EG1 data. A generalization of the GDH integral, $I_{G D H}$, to the virtual photon regime can be written as:

$$
\begin{aligned}
I^{G D H} & =\int_{\nu_{0}}^{\infty}\left[\sigma_{1 / 2}\left(\nu, Q^{2}\right)-\sigma_{3 / 2}\left(\nu, Q^{2}\right)\right] \frac{d \nu}{\nu} \\
& =\int_{0}^{x_{0}} \frac{g_{1}\left(x, Q^{2}\right)-\frac{Q^{2}}{\nu^{2}} g_{2}\left(x, Q^{2}\right)}{Q^{2} \sqrt{1+\frac{Q^{2}}{\nu^{2}}}} d x
\end{aligned}
$$

which reduces to: 


\begin{tabular}{|c|c|}
\hline$Q^{2}\left(\mathrm{GeV}^{2}\right)$ & $W_{\text {max }}(\mathrm{GeV})$ \\
\hline \hline $0.15-0.18$ & 2.0 \\
\hline $0.18-0.22$ & 2.0 \\
\hline $0.22-0.27$ & 2.1 \\
\hline $0.27-0.33$ & 2.1 \\
\hline $0.33-0.41$ & 2.6 \\
\hline $0.41-0.5$ & 2.6 \\
\hline $0.5-0.6$ & 2.5 \\
\hline $0.6-0.74$ & 2.5 \\
\hline $0.74-0.90$ & 2.4 \\
\hline $0.9-1.10$ & 2.4 \\
\hline $1.10-1.35$ & 2.3 \\
\hline $1.35-1.64$ & 2.2 \\
\hline $1.64-2.01$ & 2.2 \\
\hline
\end{tabular}

Table 5.2: Integration Limit in $\mathrm{W}$ for $Q^{2}$ bins 


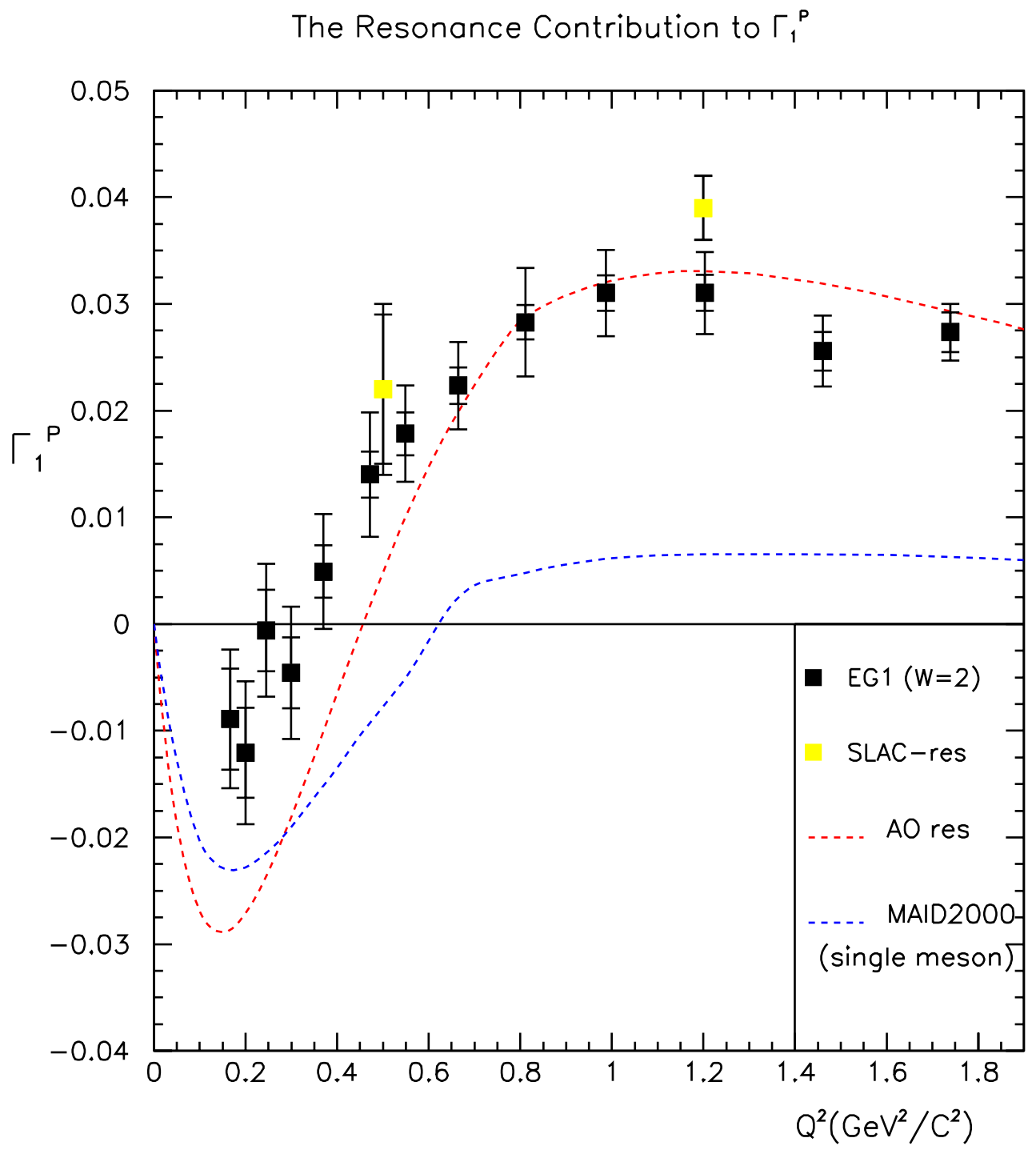

Figure 5.5: The full black squares represent the resonance contribution to $\Gamma_{1}^{P}$ for the EG1 data. 


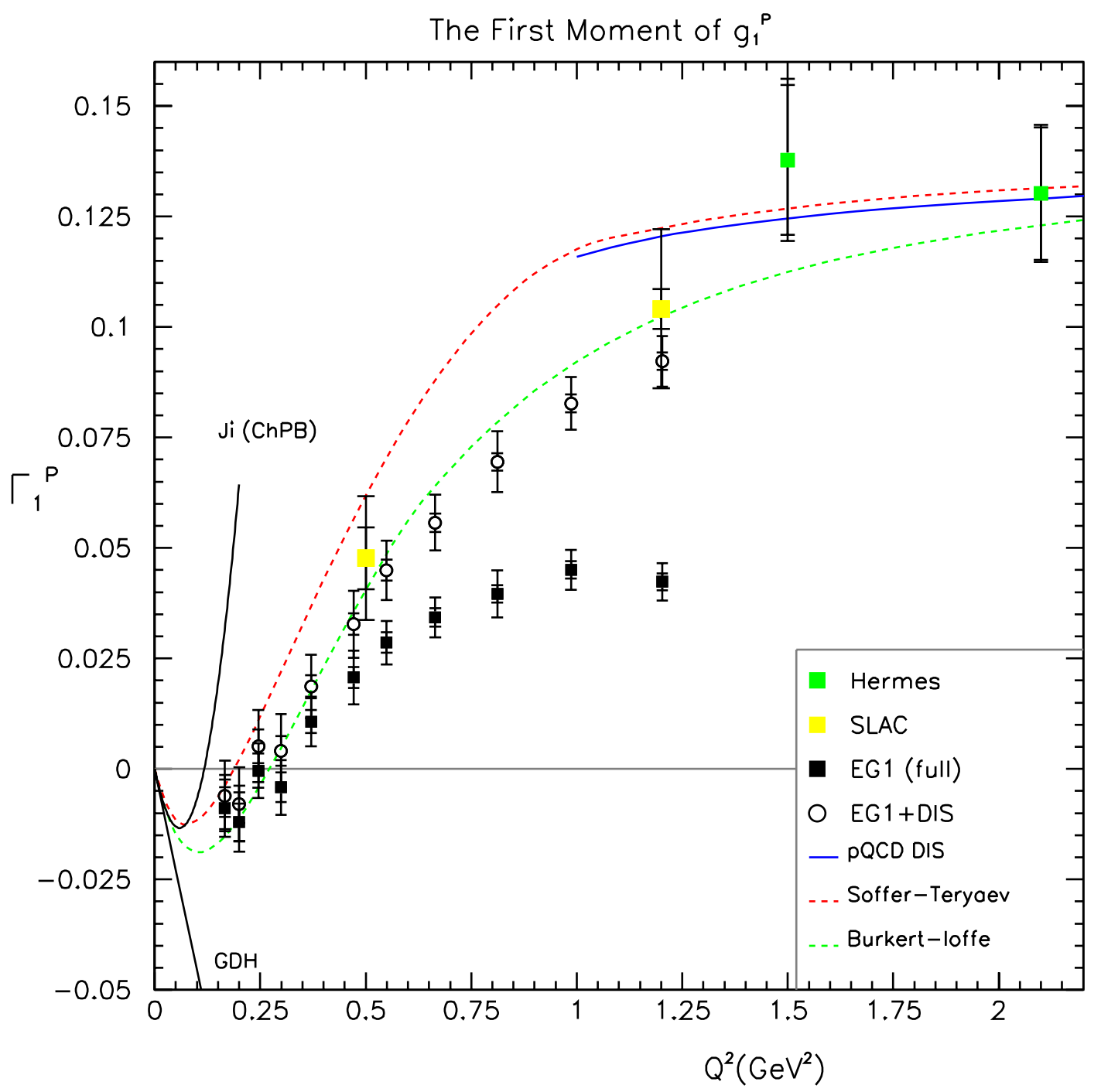

Figure 5.6: The black squares show $\Gamma_{1}^{P}$ for EG1 data integrated up to the values in $\mathrm{W}$ shown in table 5.2. The open circles represent the EG1 data plus the deep inelastic contribution given by "Models" 


$$
I_{1}^{G D H}=\int_{0}^{x_{0}} \frac{A_{1}(x) F_{1}(x)}{x K} d x
$$

where $K=\sqrt{\nu^{2}+Q^{2}}$ and $x_{0}$ is the pion threshold. In this analysis the generalized GDH integral was calculated by combining the virtual photon asymmetry, $A_{1}$, from each of the data settings for all $Q^{2}$ and $\mathrm{W}<2 \mathrm{GeV}$. Parameterizations for $F_{1}$ as well as the deep inelastic contribution to $A_{1}$ were obtained from "Models". The lowest $Q^{2}$ point reached with this data set is $0.165 \mathrm{GeV}^{2}$. Figure 5.7 shows the EG1 results for the Generalized GDH Sum Rule. The data are not in contradiction with the GDH prediction at the photon point, $-205 \mu b$. It is clear however, that significantly lower $Q^{2}$ data points will be needed in order draw definite conclusions. The crossing point for the total integral was found by a $6^{\text {th }}$ order polynomial fit to be at $Q^{2}=0.38 \mathrm{GeV}^{2}$.

The definition in Eq. (5.8) subtracts out any contribution from $\sigma_{L T}$, which disappears completely as $Q^{2} \rightarrow 0$. The longitudinal-transverse interference term is not small however. This is to be expected because of the increasingly large contribution from $A_{2}$ at low $Q^{2}$. In order to demonstrate this another generalized GDH integral has been calculated using only the values of $g_{1}$ extracted from our data.

$$
\begin{gathered}
I_{2}^{G D H}=\frac{16 \pi^{2} \alpha}{Q^{2}} \int_{0}^{x_{0}} g_{1} d x \\
I_{2}^{G D H}=\int_{0}^{x_{0}} \frac{1-x}{1-\frac{Q^{2}}{\nu^{2}}}\left[\sigma_{1 / 2}-\sigma_{3 / 2}-\frac{Q}{\nu} \sigma_{L T}\right] \frac{d \nu}{\nu}
\end{gathered}
$$

$I_{2}^{G D H} \rightarrow-\frac{2 \pi^{2} \alpha \kappa_{N}^{2}}{M^{2}}$, the real photon GDH integral value, as $Q^{2} \rightarrow 0$. A $6^{t h}$ order polynomial fit to Fig 5.8 shows the zero crossing has decreased to $Q^{2}=0.25 \mathrm{GeV}$. 
The Generalized GDH Integral I

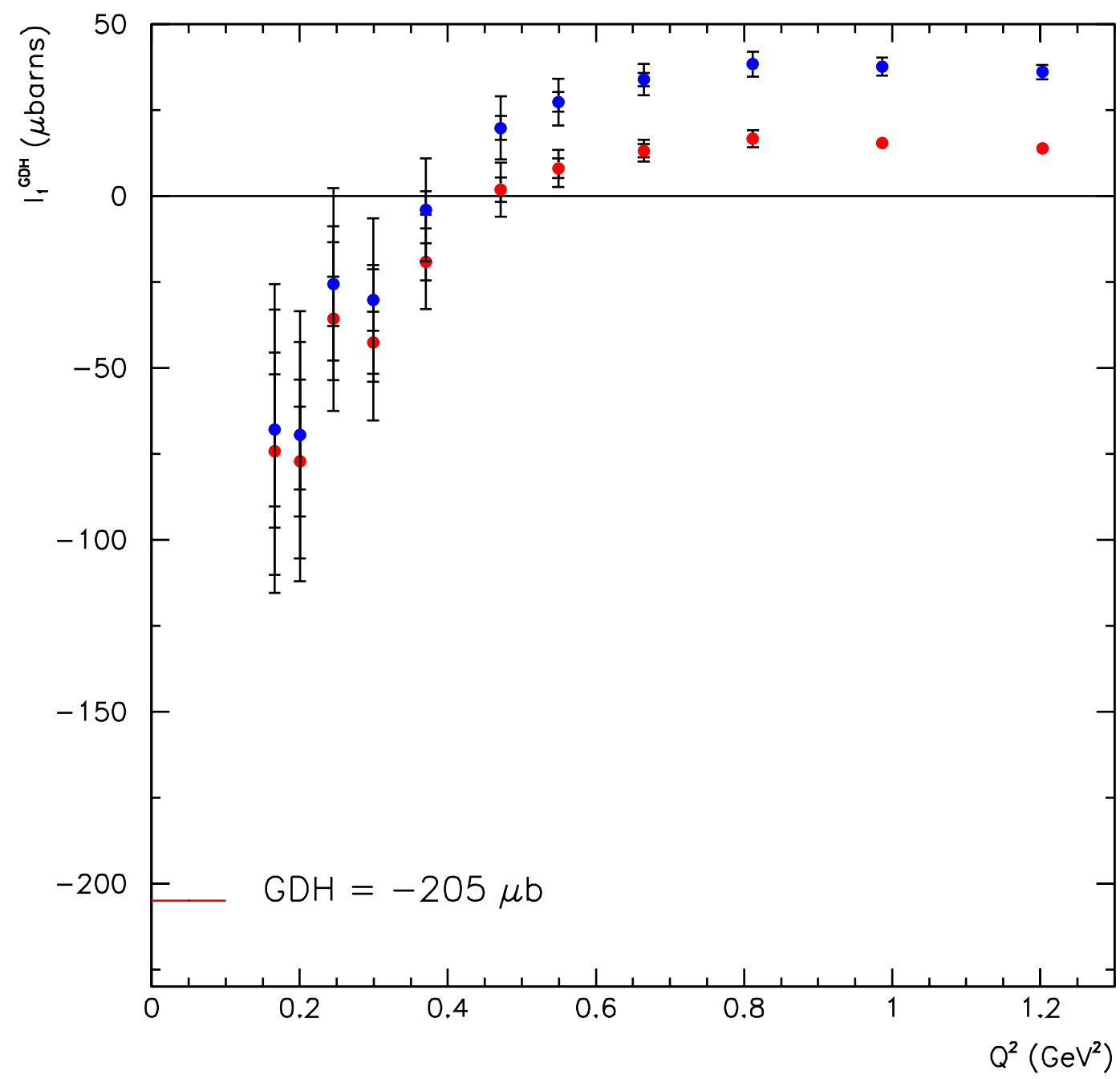

Figure 5.7: The Generalized Gerasimov-Drell-Hearn Integral for the EG1 data. The red points correspond to integration from pion threshold to the full $\mathrm{W}$ phase space available. The blue points contain the deep inelastic contribution to the integral with parameterizations from "Models". 


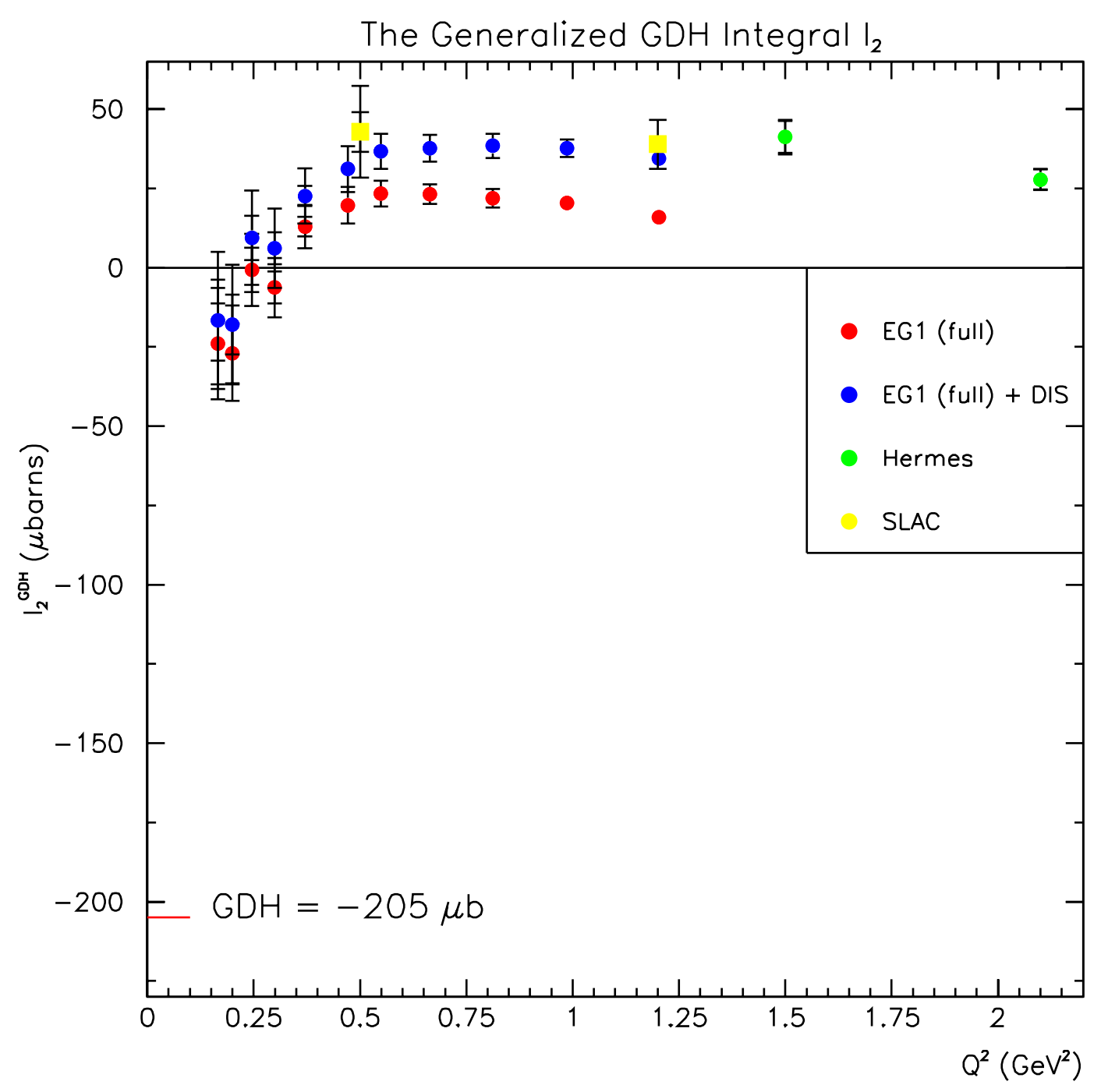

Figure 5.8: The Generalized Gerasimov-Drell-Hearn Integral with the $\sigma_{L T}$ contribution included. The red points correspond to only the resonance region and the blue points include the deep inelastic contribution. The Hermes[29] (green) and SLAC[21] (yellow) data for the first moment have been transformed to $I_{2}^{G D H}$ and are shown on the plot above. The crossing point now has a value of $W^{2}=0.25 \mathrm{GeV}^{2}$ 
Rewriting $I_{2}^{G D H}$ in terms of photo-absorption cross-sections and comparing Figs. 5.8 and 5.7 demonstrates that the $\sigma_{L T}$ term contributes significantly to the Generalized GDH Integral at the lower $Q^{2}$ points. 


\section{Chapter 6}

\section{Conclusion}

The first run of experiment 91-023 (EG1) successfully measured the $Q^{2}$ evolution

of the virtual photon asymmetries $A_{1}+\eta A_{2}$, the structure function $g_{1}^{P}$, and the first moment of the proton, $\Gamma_{1}^{P}$, for the $Q^{2}$ range $0.15-2.0 \mathrm{GeV}$. The virtual photon asymmetries and $g_{1}$ show considerable structure at low $Q^{2}$, largely due to negative contributions from the $\triangle(1232)$ resonance. As expected, the higher resonances become more excited as $Q^{2}$ increases, while the lower ones become suppressed. The first moment also reflects the increasing $\triangle$ contribution at low $Q^{2}$, eventually becoming totally negative at $Q^{2}<0.24 \mathrm{GeV}^{2}$. The values of $\Gamma_{1}^{P}$ at high $Q^{2}$ disagree with perturbative $\mathrm{QCD}[38]$ predictions, while at the same time remaining consistent with SLAC data [21] in the same $Q^{2}$ region.

EG1 has provided the highest resolution spin structure data in the resonance region to date. However, the second run of 91-023 (EG2000) has taken more than eight times the amount of data available to EG1. Increased statistical accuracy provides the opportunity for the kinematic separation of asymmetries $A_{1}$ and $A_{2}$. This separation, 
the importance of which cannot be emphasized enough, will provide some of the world's first data on $A_{2}$ in the resonance region. This in turn will produce more accurate extractions of $g_{1}, \Gamma_{1}$, and the Generalized GDH sum Rule, while eliminating the largest single systematic error present in the EG1 analysis. The independent extraction of $A_{2}$ should be the primary focus of the EG2000 analysis. Secondary areas of development include the testing of duality in spin structure functions and the calculation of higher moments of $g_{1}$ in order to extract higher twist terms.

EG2000 also has the capability of improving several analysis techniques. The background subtraction should be more accurate due to the use of frozen ${ }^{15} \mathrm{~N}$ target scattering data. In addition data from EG1 will provide stronger constraints in the resonance region for the "Models" fits. This in turn will allow a more accurate determination of radiative corrections, especially in the region of pion threshold. Finally the range of beam energies used during EG2000 will extend the measurement of all spin structure quantities to lower and higher $Q^{2}$, allowing more rigorous testing of the Generalized GDH Sum Rule as well as pQCD predictions which extend to lower $Q^{2}$ 


\section{Appendix A}

\section{Error Analysis}

\begin{tabular}{|c|c|}
\hline Symbol & Description \\
\hline \hline$\eta^{+}$ & number of counts in one bin of the $\mathrm{W}^{+}$spectra \\
\hline$\eta^{-}$ & number of counts in one bin of the $\mathrm{W}^{-}$spectra \\
\hline$F C^{+}$ & number of Faraday Cup counts for + helicity state \\
\hline$F C^{-}$ & number of Faraday Cup counts for - helicity state \\
\hline $\mathrm{R}$ & ratio of $\sigma_{N}$ to $\sigma_{P}$ \\
\hline
\end{tabular}

The numerator of $\mathrm{NH}_{3}, \mathrm{C}_{12}$ and proton asymmetry is:

$$
N=\frac{\eta^{+}}{F C^{+}}-\frac{\eta^{-}}{F C^{-}}
$$

The denominator of $\mathrm{NH}_{3}, \mathrm{C}_{12}$ and proton asymmetry is:

$$
D=\frac{\eta^{+}}{F C^{+}}+\frac{\eta^{-}}{F C^{-}}
$$


The error for $\mathrm{N}$ and $\mathrm{D}$ :

$$
\triangle N=\triangle D=\sqrt{\frac{\eta^{+}}{F C^{+2}}+\frac{\eta^{-}}{F C^{-2}}}
$$

The proton asymmetry $A_{P}$ is defined as follows:

$$
A_{P}=\frac{N_{N H_{3}}}{D_{N H_{3}}-D_{N 15}}=\frac{N_{N H_{3}}}{D_{P}}
$$

The error for the proton asymmetry is calculated as follows:

$$
\triangle A_{P}=\frac{1}{D_{P}} \sqrt{A_{P}{ }^{2} Z^{2} \triangle D_{C_{12}}{ }^{2}+\left(1-A_{P}\right)^{2} \frac{\eta_{N H_{3}}^{+}}{F C^{+2}}+\left(1+A_{P}\right)^{2} \frac{\eta_{N H_{3}}^{-}}{F C^{-2}}}
$$

The constant $\mathrm{Z}$ is the ratio of the ${ }^{15} \mathrm{~N} /{ }^{12} \mathrm{C}$ spectra. $D_{C_{12}}$, the carbon spectra, is multiplied by $\mathrm{Z}$ in order to reconstruct the ${ }^{15} \mathrm{~N}$ background. The length of the $\mathrm{NH}_{3}$ target, $\chi_{N H_{3}}$, is embedded in $\mathrm{Z}$.

$$
Z=\frac{\left(\frac{7+8 R}{9+9 R}\right) \rho_{N H_{3}} \chi_{N H_{3}}+\rho_{H e}\left(L-\chi_{N H_{3}}\right)+\rho_{A l} \chi_{A l}+\rho_{K} \chi_{K}}{\rho_{C_{12}} \chi_{C_{12}}+\rho_{H e}\left(L-\chi_{C_{12}}\right)+\rho_{A l} \chi_{A l}+\rho_{K} \chi_{K}}
$$

The statistical error on the Faraday Cup readings, FC, is defined as [83]:

$$
\sigma=\frac{\sqrt{2}}{20 \sqrt{n} I}
$$

where $\mathrm{n}$ is the number of helicity pairs and $\mathrm{I}$ is the average current for that setting. Table A.1 details the Faraday cup asymmetry error for each setting. This error is several orders of magnitude smaller than the statistical errors outlined above and therefore was not included in the complete statistical error calculation. 


\begin{tabular}{|c|c|c|c|c|c|}
\hline Beam $(\mathrm{GeV})$ & Torus $(\mathrm{A})$ & Target Pol & $\mathrm{I}(\mathrm{nA})$ & $\mathrm{n}$ & $\sigma_{A_{F C}} n A^{-1}$ \\
\hline \hline 2.562 & -1500 & plus & 0.5 & 33647 & 0.00077 \\
\hline 2.562 & -1500 & minus & 0.5 & 47620.5 & 0.00065 \\
\hline 2.565 & 2250 & plus & 2.0 & 41586.5 & 0.00029 \\
\hline 2.565 & 2250 & minus & 2.0 & 35323 & 0.00019 \\
\hline 2.565 & 1500 & plus & 1.8 & 12472 & 0.00035 \\
\hline 2.565 & 1500 & minus & 1.8 & 26560 & 0.00024 \\
\hline 4.278 & 2250 & plus & 3.8 & 43608.5 & 0.000089 \\
\hline 4.278 & 2250 & minus & 3.8 & 314245 & 0.000033 \\
\hline 4.278 & -2250 & plus & 1.9 & 23394 & 0.00024 \\
\hline 4.278 & -2250 & minus & 1.9 & 39164 & 0.00019 \\
\hline
\end{tabular}

Table A.1: The Faraday Cup asymmetry error for individual beam and target settings.

Once the proton asymmetry has been extracted the product of the beam and target polarization is calculated. This calculation results in a combination of statistical and systematic errors. The statistical error comes from $\triangle A_{p}$ in the elastic peak and from the subsequent fit to the $P_{b} P_{t}$ vs. $Q^{2}$ distribution. This statistical error has not been incorporated into the total error in this analysis. The systematic error associated with this calculation comes from the error of the theoretical calculation of the elastic asymmetry, $A_{p}$, as well as the systematic error inherent in the extraction of the proton elastic peak.

$$
\triangle P_{b} P_{t}=P b P t * \frac{\triangle A_{p}^{\text {sum }}}{A_{p}^{\text {sum }}}
$$

$A_{p}^{\text {sum }}$ is the statistically weighted sum, and $\triangle A_{p}^{\text {sum }}$ is the error of the two bins which make up the elastic peak. These two bins span a $\mathrm{W}$ range of $0.92-0.96 \mathrm{GeV}$. The error values quoted in the text are from a zeroth order fit to several $P_{b} P_{t}$ values. The 
errors on the individual values are calculated in the above manner.

After the asymmetry is corrected for the beam and target polarizations the pair symmetric background correction is applied. The error corresponding to this calculation is:

$$
\triangle A_{\|}=\frac{1}{1-R_{r}} \sqrt{\triangle A_{p}^{2}+A_{p}^{2} R_{r}^{2} \triangle R_{a}^{2}+\frac{A_{p}^{2} \triangle R_{r}^{2}}{\left(1-R_{r}\right)^{2}}}
$$

$\triangle A_{\|}$is the error on the corrected asymmetry, $\triangle A_{p}$ is the error on the raw asymmetry, $R_{r}$ is the measured ratio of positrons to electrons, and $R_{a}$ is the ratio of positron-electron raw asymmetries.

Radiative corrections are applied after the pair symmetric correction and there are no statistical errors associated with these corrections. There is no multiplicative factor used in this version of radiative corrections so the errors are identical to the ones beforehand.

After radiative corrections are implemented the statistical errors are only affected by the act of combining $\mathrm{W}$ and $Q^{2}$ bins or multiplication by factors.

$$
\begin{aligned}
& \triangle\left(\frac{A_{\|}}{D}\right)=\frac{\triangle A_{\|}}{D}=\triangle A_{1} \\
& \triangle g_{1}=\triangle A_{1} *\left(\frac{F_{1} \tau}{\tau+1}\right)
\end{aligned}
$$

The manipulation from the experimental asymmetry, $A_{\|}$, to $A_{\|} / D, A_{1}$ and $g_{1}$ requires the use of models from Strucfunc.F, the errors from which are accounted for in the systematic error analysis.

The first moment calculation requires each $\mathrm{W}$ bin of $g_{1}$ to be multiplied by a 
Jacobian, $J_{W}$, and then added together. The corresponding error calculation is:

$$
\triangle \Gamma_{1}^{P}=\sqrt{\sum_{W}\left(\triangle g_{1_{W}} * J_{W}^{i n t}\right)^{2}}
$$

The same procedure is used for the calculation of the GDH integral error.

$$
\triangle G D H=\sqrt{\sum_{W}\left(\triangle A_{1_{W}} * J_{W}^{G D H}\right)^{2}}
$$

Notice the Jacobians for the first moment and GDH integral are different and are defined in the main text. 


\section{Appendix B}

\section{GDH derivation}

The forward Compton scattering amplitude of a real photon on a nucleon is:

$$
F(\nu, \theta=0)=\chi_{f}^{\dagger}\left[\overrightarrow{\epsilon_{f}^{*}} \cdot \vec{\epsilon}_{i} f_{1}(\nu)+i \vec{\sigma} \cdot\left(\overrightarrow{\epsilon_{f}^{*}} \times \overrightarrow{\epsilon_{i}}\right) f_{2}(\nu)\right] \chi_{i}
$$

where $\chi_{i(f)}$ are the initial (final) Pauli spinors, $\epsilon_{i(f)}$ denote the polarization vectors of the inital and final photon and $\vec{\sigma}$ is the spin of the nucleon. The functions $f_{1}(\nu)$ and $f_{2}(\nu)$ refer to the spin non-flip and flip amplitudes, which can be determined by looking at at the $F_{3 / 2}$ and $F_{1 / 2}$ amplitudes. $F_{3 / 2}$ refers to a spin 1 photon scattering from an aligned spin $\frac{1}{2}$ nucleon and $F_{1 / 2}$ from an anti-aligned nucleon. This results in the following expressions:

$$
F_{3 / 2}=f_{1}-f_{2} \quad F_{1 / 2}=f_{1}+f_{2}
$$


Solving for $f_{1}$ and $f_{2}$ results in

$$
f_{1}=\frac{\left(F_{3 / 2}+F_{1 / 2}\right)}{2} \quad f_{2}=\frac{\left(F_{1 / 2}-F_{3 / 2}\right)}{2}
$$

As in the previous section the optical theorem can be used to relate the imaginary parts of $F_{3 / 2}, F_{1 / 2}$ to the total Compton crossections.

$$
\Im f_{1}(\nu)=\frac{\nu}{8 \pi}\left(\sigma_{1 / 2}+\sigma_{3 / 2}\right) \quad \Im f_{2}(\nu)=\frac{\nu}{8 \pi}\left(\sigma_{1 / 2}-\sigma_{3 / 2}\right)
$$

At this point it is possible to write down a dispersion relationship between the imaginary and real parts of $f_{2}$. It is of course also possible for $f_{1}$ but for the GDH we only need to focus on the latter. $f_{2}(\nu)$ is an analytical function in the region of the positive imaginary axis and therefore can be expressed using the Cauchy Integral Formulas.

$$
f_{2}(\nu)=\frac{1}{2 i \pi} \oint_{C} \frac{f_{2}(z) d z}{z-\nu} \Rightarrow \frac{1}{2 i \pi} \int_{-\infty}^{+\infty} \frac{f_{2}(z) d z}{z-\nu}
$$

If it is true that $f_{2}(z)$ goes to zero when z goes to $\infty$ faster than $\frac{1}{z}$ then the contribution from the half circle part of the contour vanishes leaving only an integration of the real axis. Rewriting $f_{2}$ as

$$
f_{2}(\nu)=\lim _{\epsilon \rightarrow 0} f_{2}(\nu+i \epsilon)
$$

and using the Cauchy relation

$$
\lim _{\epsilon \rightarrow 0} \frac{1}{x \pm i \epsilon}=\mathcal{P}\left(\frac{1}{x}\right) \mp i \pi \delta(x)
$$


allows equation B.5 to be expressed as:

$$
f_{2}(\nu)=\lim _{\epsilon \rightarrow 0} \frac{1}{2 i \pi} \int_{-\infty}^{+\infty} \frac{f_{2}(z)}{z-(\nu+i \epsilon)} d \nu=\frac{1}{2 i \pi} \mathcal{P} \int_{-\infty}^{+\infty} \frac{f_{2}(z)}{z-\nu}+\frac{1}{2} f_{2}(\nu)
$$

The dispersion relation between the imaginary and real parts of the above expression is written as:

$$
\Re f_{2}(\nu)=\frac{1}{\pi} \mathcal{P} \int_{-\infty}^{+\infty} \frac{\Im f_{2}(z)}{z-\nu} d z=\frac{2 \nu}{\pi} \mathcal{P} \int_{0}^{+\infty} \frac{\Im f_{2}(z)}{z^{2}-\nu^{2}} d z
$$

Referring back to the expression for imaginary amplitudes that was obtained using the optical theorem it is clear that

$$
\Re f_{2}(\nu)=\frac{\nu^{2}}{8 \pi} \mathcal{P} \int_{0}^{+\infty} \frac{\sigma_{1 / 2}-\sigma_{3 / 2}}{z^{2}-\nu^{2}} d z
$$

Gell-Mann, Goldberger and Low [26] showed that $f_{2}(\nu)$ can be expanded into a power series for small $\nu$, where the leading terms are determined by low energy theorems which are based only on Lorentz and gauge invariance.

$$
f_{2}(\nu)=-\frac{e^{2} \kappa^{2}}{2 m^{2}} \nu+\gamma_{N} \nu^{3}+\mathcal{O}\left(\nu^{5}\right)
$$

The final step requires expanding the denominator of equation B.10, differentiating with respect to $\nu$, setting $\nu=0$ and then equating it to equation ?? which is also differentiated.

$$
f_{2}(\nu)=\frac{1}{4 \pi^{2}} \int_{0}^{\infty}\left(\sigma_{1 / 2}-\sigma_{3 / 2}\right)\left(\frac{\nu}{z}+\frac{\nu^{3}}{z^{3}}+\ldots\right) d z
$$




$$
\left(\frac{\partial}{\partial \nu}\right)\left[f_{2}(\nu)\right]_{\nu=0}=\frac{1}{4 \pi^{2}} \int_{0}^{\infty} \frac{\sigma_{1 / 2}-\sigma_{3 / 2}}{z} d z=-\frac{\alpha \kappa^{2}}{2 m^{2}}
$$

The only weakness in the derivation is the assumption of an unsubtracted dispersion relation. In equation B.10 there is a constant that all standard derivations assume to be zero. The "subtracted" dispersion relation takes the following form.

$$
\Re f_{2}(\nu)=\Re\left(f_{2}(0)\right)+\frac{\nu}{\pi} \mathcal{P} \int_{-\infty}^{\infty} \frac{\Im f_{2}(z)}{z(z-\nu)} d z
$$

It is often argued in the literature that this term exists in order to make the integral more convergent. If a subtraction is necessary to make the integral convergent then it would appear in the form of this constant. However it is completely possible that even if the integral converges the constant could be non-zero and hence violate the sum rule. This is also why high energy measurements of $\sigma_{3 / 2}$ and $\sigma_{1 / 2}$ cannot determine the existence of the constant. 


\section{Appendix $\mathrm{C}$}

\section{Data Tables}

The following tables contain data points as well as statistical and systematic errors

for the plots of $A_{1}+\eta A_{2}, g_{1}^{P}$ vs $\mathrm{X}_{B J}, \Gamma_{1}^{P}$ and the Generalized GDH integral $I_{1}$ found in Chapter 5. 
Table C.1: $A_{1}+\eta A_{2}$ for $Q^{2}=0.15-0.22 \mathrm{GeV}^{2}$ (see Fig. 5.1).

\begin{tabular}{|cccc|}
\hline $\mathrm{W}(\mathrm{GeV})$ & $A_{1}+\eta A_{2}$ & $\sigma$ & sys \\
\hline 1.10000 & $-0 . .40921$ & 0.21042 & 0.04056 \\
1.14000 & $-0 . .39048$ & 0.20573 & 0.04905 \\
1.18000 & $-0 . .45321$ & 0.11246 & 0.06936 \\
1.22000 & $-0 . .30241$ & 0.08677 & 0.04930 \\
1.26000 & $-0 . .25552$ & 0.08974 & 0.04057 \\
1.30000 & $-0 . .07544$ & 0.09706 & 0.01276 \\
1.34000 & 0.14489 & 0.10485 & 0.01904 \\
1.38000 & 0.13396 & 0.09028 & 0.01303 \\
1.42000 & $-0 . .05836$ & 0.08969 & 0.00811 \\
1.46000 & 0.12695 & 0.07233 & 0.01190 \\
1.50000 & 0.01482 & 0.05576 & 0.00432 \\
1.54000 & 0.09313 & 0.05601 & 0.00901 \\
1.58000 & 0.11333 & 0.05612 & 0.00743 \\
1.62000 & 0.00855 & 0.05649 & 0.00415 \\
1.66000 & 0.02426 & 0.05103 & 0.00465 \\
1.70000 & 0.02180 & 0.04610 & 0.00339 \\
1.74000 & 0.02286 & 0.04885 & 0.00341 \\
1.78000 & 0.04486 & 0.04668 & 0.00364 \\
1.82000 & 0.08536 & 0.04567 & 0.00522 \\
1.86000 & $-0 . .02744$ & 0.04282 & 0.00259 \\
1.90000 & $-0 . .04532$ & 0.04618 & 0.00471 \\
1.94000 & $-0 . .06566$ & 0.04583 & 0.00451 \\
1.98000 & 0.00765 & 0.05108 & 0.00408 \\
2.02000 & $-0 . .00374$ & 0.03690 & 0.00259 \\
\hline .06000 & 0.09868 & 0.03648 & 0.00654 \\
\hline
\end{tabular}


Table C.2: $A_{1}+\eta A_{2}$ for $Q^{2}=0.22-0.41 \mathrm{GeV}^{2}$ (see Fig. 5.1).

\begin{tabular}{|cccc|}
\hline $\mathrm{W}(\mathrm{GeV})$ & $A_{1}+\eta A_{2}$ & $\sigma$ & sys \\
\hline 1.10000 & $-0 . .66645$ & 0.25542 & 0.11961 \\
1.14000 & $-0 . .82496$ & 0.22236 & 0.15005 \\
1.18000 & $-0 . .54109$ & 0.13081 & 0.12525 \\
1.22000 & $-0 . .41558$ & 0.09000 & 0.09236 \\
1.26000 & $-0 . .24481$ & 0.08827 & 0.05128 \\
1.30000 & $-0 . .04955$ & 0.10093 & 0.01875 \\
1.34000 & 0.06689 & 0.09752 & 0.01790 \\
1.38000 & 0.15684 & 0.10227 & 0.02567 \\
1.42000 & 0.10132 & 0.08680 & 0.01740 \\
1.46000 & 0.19359 & 0.07489 & 0.02592 \\
1.50000 & 0.46181 & 0.06400 & 0.06822 \\
1.54000 & 0.28113 & 0.05825 & 0.03338 \\
1.58000 & 0.24518 & 0.05989 & 0.02339 \\
1.62000 & 0.08081 & 0.05550 & 0.01143 \\
1.66000 & 0.13447 & 0.04877 & 0.01524 \\
1.70000 & 0.12112 & 0.04196 & 0.01390 \\
1.74000 & 0.16024 & 0.04541 & 0.01481 \\
1.78000 & 0.15749 & 0.04414 & 0.01387 \\
1.82000 & 0.02837 & 0.03970 & 0.00895 \\
1.86000 & 0.09967 & 0.03819 & 0.01181 \\
1.90000 & 0.06535 & 0.03396 & 0.00900 \\
1.94000 & 0.06235 & 0.03179 & 0.00938 \\
1.98000 & 0.02580 & 0.03018 & 0.00676 \\
2.02000 & 0.04720 & 0.02682 & 0.00731 \\
& 0.05343 & 0.02584 & 0.00796 \\
\hline
\end{tabular}


Table C.3: $A_{1}+\eta A_{2}$ for $Q^{2}=0.41-0.6 \mathrm{GeV}^{2}$ (see Fig. 5.1).

\begin{tabular}{|cccc|}
\hline $\mathrm{W}(\mathrm{GeV})$ & $A_{1}+\eta A_{2}$ & $\sigma$ & sys \\
\hline 1.10000 & $-0 . .73324$ & 0.17210 & 0.13492 \\
1.14000 & $-0 . .17849$ & 0.15956 & 0.07112 \\
1.18000 & $-0 . .31860$ & 0.09550 & 0.07298 \\
1.22000 & $-0 . .40073$ & 0.05995 & 0.08615 \\
1.26000 & $-0 . .22546$ & 0.06344 & 0.04422 \\
1.30000 & $-0 . .04195$ & 0.07591 & 0.01668 \\
1.34000 & 0.18343 & 0.07818 & 0.02667 \\
1.38000 & 0.37267 & 0.07118 & 0.04677 \\
1.42000 & 0.33113 & 0.06915 & 0.03431 \\
1.46000 & 0.34712 & 0.05834 & 0.03799 \\
1.50000 & 0.32417 & 0.04429 & 0.04366 \\
1.54000 & 0.46256 & 0.04524 & 0.05179 \\
1.58000 & 0.37110 & 0.05028 & 0.03185 \\
1.62000 & 0.15281 & 0.04518 & 0.01443 \\
1.66000 & 0.23745 & 0.03899 & 0.02206 \\
1.70000 & 0.14723 & 0.03202 & 0.01690 \\
1.74000 & 0.23199 & 0.03335 & 0.01630 \\
1.78000 & 0.23101 & 0.03515 & 0.01739 \\
1.82000 & 0.11878 & 0.03288 & 0.01161 \\
1.86000 & 0.12020 & 0.03040 & 0.01330 \\
1.90000 & 0.12189 & 0.02811 & 0.01577 \\
1.94000 & 0.08679 & 0.02637 & 0.01265 \\
1.98000 & 0.04604 & 0.02549 & 0.00890 \\
2.02000 & 0.08966 & 0.02954 & 0.01074 \\
2.06000 & 0.14069 & 0.03528 & 0.02071 \\
& & & \\
& & \\
& & \\
1.300
\end{tabular}


Table C.4: $A_{1}+\eta A_{2}$ for $Q^{2}=0.6-1.1 \mathrm{GeV}^{2}$ (see Fig. 5.1).

\begin{tabular}{|cccc|}
\hline $\mathrm{W}(\mathrm{GeV})$ & $A_{1}+\eta A_{2}$ & $\sigma$ & sys \\
\hline 1.10000 & $-0 . .33236$ & 0.14255 & 0.07313 \\
1.14000 & $-0 . .32348$ & 0.13062 & 0.07000 \\
1.18000 & $-0 . .22582$ & 0.06914 & 0.04256 \\
1.22000 & $-0 . .34372$ & 0.05015 & 0.06911 \\
1.26000 & $-0 . .12200$ & 0.05052 & 0.03147 \\
1.30000 & 0.02478 & 0.05555 & 0.01183 \\
1.34000 & 0.34310 & 0.05823 & 0.04218 \\
1.38000 & 0.22665 & 0.05951 & 0.02672 \\
1.42000 & 0.30072 & 0.05932 & 0.03201 \\
1.46000 & 0.50152 & 0.04696 & 0.05252 \\
1.50000 & 0.49644 & 0.03780 & 0.05225 \\
1.54000 & 0.51128 & 0.03728 & 0.04376 \\
1.58000 & 0.37496 & 0.03784 & 0.02955 \\
1.62000 & 0.39731 & 0.03801 & 0.03397 \\
1.66000 & 0.24307 & 0.03013 & 0.01871 \\
1.70000 & 0.32773 & 0.02733 & 0.02641 \\
1.74000 & 0.29672 & 0.02744 & 0.02193 \\
1.78000 & 0.27432 & 0.02912 & 0.02492 \\
1.82000 & 0.23148 & 0.03031 & 0.02538 \\
1.86000 & 0.15189 & 0.02737 & 0.02746 \\
1.90000 & 0.10823 & 0.03962 & 0.02445 \\
& & & \\
1.50
\end{tabular}


Table C.5: $g_{1}^{P}$ vs. $\mathrm{X}_{B J}$ for $Q^{2}=0.15-0.27 \mathrm{GeV}^{2}$ (see Fig. 5.3).

\begin{tabular}{|cccc|cccc|}
\hline$X_{B J}$ & $g_{1}^{P}$ & $\sigma$ & sys & $X_{B J}$ & $g_{1}^{P}$ & $\sigma$ & sys \\
\hline 0.37800 & -0.00252 & 0.00176 & 0.00282 & 0.10311 & 0.04932 & 0.03420 & 0.01817 \\
0.32338 & -0.01978 & 0.01162 & 0.00802 & 0.09659 & 0.03078 & 0.03845 & 0.01538 \\
0.28129 & -0.10720 & 0.02395 & 0.02400 & 0.09072 & 0.07318 & 0.04005 & 0.01573 \\
0.24791 & -0.22704 & 0.04148 & 0.06114 & 0.08541 & 0.07023 & 0.03502 & 0.01490 \\
0.22083 & -0.12772 & 0.03813 & 0.03937 & 0.08057 & 0.07879 & 0.03374 & 0.01697 \\
0.19845 & 0.02072 & 0.03360 & 0.01876 & 0.07617 & 0.09361 & 0.03275 & 0.02091 \\
0.17968 & 0.06765 & 0.02980 & 0.01852 & 0.07213 & 0.00342 & 0.03365 & 0.01957 \\
0.16372 & 0.08753 & 0.02815 & 0.01806 & 0.06843 & 0.00014 & 0.03884 & 0.02200 \\
0.15000 & 0.03162 & 0.03003 & 0.01937 & 0.06502 & -0.03899 & 0.03768 & 0.02324 \\
0.13810 & 0.10544 & 0.03385 & 0.02305 & 0.06188 & 0.08171 & 0.03927 & 0.02235 \\
0.12769 & 0.09510 & 0.04062 & 0.02627 & 0.05897 & 0.00822 & 0.03371 & 0.01465 \\
0.11851 & 0.12188 & 0.03639 & 0.02427 & 0.05627 & 0.10099 & 0.03820 & 0.02255 \\
0.11037 & 0.11646 & 0.03185 & 0.02371 & 0.05376 & 0.00362 & 0.08703 & 0.02335 \\
\hline
\end{tabular}


Table C.6: $g_{1}^{P}$ vs. $\mathrm{X}_{B J}$ for $Q^{2}=0.27-0.5 \mathrm{GeV}^{2}$ (see Fig. 5.3).

\begin{tabular}{|cccc|cccc|}
\hline$X_{B J}$ & $g_{1}^{P}$ & $\sigma$ & sys & $X_{B J}$ & $g_{1}^{P}$ & $\sigma$ & sys \\
\hline 0.53167 & -0.00368 & 0.00081 & 0.00220 & 0.12681 & 0.08566 & 0.01624 & 0.03169 \\
0.47169 & -0.01684 & 0.00535 & 0.00596 & 0.12067 & 0.07764 & 0.01541 & 0.02278 \\
0.42234 & -0.10422 & 0.01216 & 0.02242 & 0.11498 & 0.08984 & 0.01577 & 0.01978 \\
0.38110 & -0.14404 & 0.01786 & 0.03749 & 0.10970 & 0.07338 & 0.01628 & 0.02417 \\
0.34617 & -0.06912 & 0.01613 & 0.02167 & 0.10479 & 0.06651 & 0.01656 & 0.00971 \\
0.31624 & -0.01868 & 0.01454 & 0.01494 & 0.10022 & 0.08450 & 0.02007 & 0.01390 \\
0.29036 & 0.03910 & 0.01355 & 0.01120 & 0.09595 & 0.09620 & 0.02911 & 0.01739 \\
0.26778 & 0.07554 & 0.01248 & 0.01229 & 0.09195 & 0.13442 & 0.05725 & 0.01532 \\
0.24793 & 0.06360 & 0.01384 & 0.01743 & 0.08821 & 0.16181 & 0.05730 & 0.01514 \\
0.23037 & 0.13367 & 0.01685 & 0.02377 & 0.08470 & 0.06171 & 0.05397 & 0.01392 \\
0.21473 & 0.21634 & 0.01974 & 0.04205 & 0.08140 & 0.18993 & 0.05960 & 0.01608 \\
0.20074 & 0.21006 & 0.01857 & 0.03077 & 0.07830 & 0.10594 & 0.05508 & 0.01419 \\
0.18816 & 0.15645 & 0.01593 & 0.03520 & 0.07537 & 0.09491 & 0.05489 & 0.01488 \\
0.17679 & 0.09755 & 0.01732 & 0.01865 & 0.07262 & 0.24176 & 0.05619 & 0.01876 \\
0.16648 & 0.12639 & 0.01964 & 0.01930 & 0.07001 & 0.06530 & 0.05742 & 0.01518 \\
0.15710 & 0.13251 & 0.02036 & 0.01889 & 0.06755 & 0.14025 & 0.05759 & 0.01673 \\
0.14853 & 0.13491 & 0.01767 & 0.02675 & 0.06521 & 0.00741 & 0.05904 & 0.01693 \\
0.14068 & 0.12989 & 0.01751 & 0.02080 & 0.06300 & 0.08507 & 0.06056 & 0.01820 \\
0.13346 & 0.07669 & 0.01599 & 0.02056 & 0.06091 & 0.17458 & 0.05619 & 0.02525 \\
\hline
\end{tabular}


Table C.7: $g_{1}^{P}$ vs. $\mathrm{X}_{B J}$ for $Q^{2}=0.5-0.74 \mathrm{GeV}^{2}$ (see Fig. 5.3).

\begin{tabular}{|cccc|cccc|}
\hline$X_{B J}$ & $g_{1}^{P}$ & $\sigma$ & sys & $X_{B J}$ & $g_{1}^{P}$ & $\sigma$ & sys \\
\hline 0.64770 & -0.00333 & 0.00069 & 0.00230 & 0.19963 & 0.10212 & 0.01480 & 0.03620 \\
0.59115 & -0.00659 & 0.00462 & 0.00394 & 0.19041 & 0.12326 & 0.01535 & 0.02488 \\
0.54213 & -0.03317 & 0.00921 & 0.00749 & 0.18183 & 0.09230 & 0.01574 & 0.03378 \\
0.49930 & -0.11502 & 0.01358 & 0.03249 & 0.17382 & 0.08709 & 0.01571 & 0.02980 \\
0.46161 & -0.05153 & 0.01287 & 0.01466 & 0.16635 & 0.08371 & 0.01611 & 0.02476 \\
0.42825 & 0.00136 & 0.01131 & 0.00584 & 0.15936 & 0.16895 & 0.03454 & 0.01359 \\
0.39854 & 0.04545 & 0.01010 & 0.00777 & 0.15281 & 0.14537 & 0.04649 & 0.01060 \\
0.37196 & 0.05422 & 0.01023 & 0.01047 & 0.14666 & 0.22070 & 0.04210 & 0.01279 \\
0.34806 & 0.06064 & 0.01151 & 0.01247 & 0.14089 & 0.10540 & 0.04958 & 0.01046 \\
0.32648 & 0.12392 & 0.01335 & 0.01671 & 0.13545 & 0.16103 & 0.04917 & 0.01121 \\
0.30693 & 0.20979 & 0.01644 & 0.03143 & 0.13033 & 0.23510 & 0.04945 & 0.01415 \\
0.28914 & 0.18600 & 0.01505 & 0.02176 & 0.12550 & 0.26392 & 0.04809 & 0.01494 \\
0.27291 & 0.15626 & 0.01472 & 0.02184 & 0.12094 & 0.15596 & 0.04621 & 0.01135 \\
0.25805 & 0.13226 & 0.01508 & 0.02549 & 0.11662 & 0.21212 & 0.04839 & 0.01338 \\
0.24441 & 0.14270 & 0.01610 & 0.01904 & 0.11254 & 0.13602 & 0.05145 & 0.01232 \\
0.23186 & 0.16576 & 0.01679 & 0.03591 & 0.10867 & 0.23763 & 0.04854 & 0.01902 \\
0.22027 & 0.16038 & 0.01466 & 0.02682 & 0.10500 & 0.17900 & 0.04824 & 0.01820 \\
0.20956 & 0.13862 & 0.01435 & 0.03308 & 0.10151 & 0.21084 & 0.06479 & 0.02342 \\
\hline
\end{tabular}


Table C.8: $g_{1}^{P}$ vs. $\mathrm{X}_{B J}$ for $Q^{2}=0.74-1.10 \mathrm{GeV}^{2}$ (see Fig. 5.3).

\begin{tabular}{|cccc|cccc|}
\hline$X_{B J}$ & $g_{1}^{P}$ & $\sigma$ & sys & $X_{B J}$ & $g_{1}^{P}$ & $\sigma$ & sys \\
\hline 0.73373 & 0.00001 & 0.00066 & 0.00089 & 0.28437 & 0.15101 & 0.01372 & 0.02315 \\
0.68426 & -0.00802 & 0.00358 & 0.00282 & 0.27211 & 0.15178 & 0.01564 & 0.02103 \\
0.63959 & -0.03017 & 0.00686 & 0.00681 & 0.26063 & 0.11178 & 0.01939 & 0.01918 \\
0.59914 & -0.05125 & 0.00928 & 0.01052 & 0.24986 & 0.12076 & 0.01861 & 0.02443 \\
0.56238 & -0.01124 & 0.00867 & 0.00841 & 0.23974 & 0.12664 & 0.02451 & 0.01167 \\
0.52889 & 0.00917 & 0.00772 & 0.00338 & 0.23022 & 0.16779 & 0.03451 & 0.01342 \\
0.49828 & 0.03937 & 0.00678 & 0.00886 & 0.22126 & 0.18005 & 0.03487 & 0.00830 \\
0.47025 & 0.03264 & 0.00772 & 0.00701 & 0.21281 & 0.16921 & 0.03655 & 0.00975 \\
0.44450 & 0.06168 & 0.00872 & 0.00578 & 0.20483 & 0.18736 & 0.03377 & 0.01088 \\
0.42080 & 0.10370 & 0.00979 & 0.01408 & 0.19730 & 0.18929 & 0.03825 & 0.01043 \\
0.39895 & 0.18081 & 0.01270 & 0.01987 & 0.19017 & 0.19980 & 0.03504 & 0.01029 \\
0.37874 & 0.16136 & 0.01129 & 0.02696 & 0.18342 & 0.17949 & 0.03462 & 0.00990 \\
0.36002 & 0.12381 & 0.01035 & 0.02322 & 0.17702 & 0.21277 & 0.03852 & 0.01321 \\
0.34266 & 0.14039 & 0.01142 & 0.02349 & 0.17095 & 0.18162 & 0.03644 & 0.01207 \\
0.32652 & 0.11786 & 0.01321 & 0.02506 & 0.16518 & 0.11947 & 0.03521 & 0.01033 \\
0.31148 & 0.19674 & 0.01466 & 0.02239 & 0.15971 & 0.22000 & 0.03868 & 0.02044 \\
0.29746 & 0.16531 & 0.01363 & 0.01909 & & & & \\
\hline
\end{tabular}


Table C.9: $g_{1}^{P}$ vs. $\mathrm{X}_{B J}$ for $Q^{2}=1.10-1.64 \mathrm{GeV}^{2}$ (see Fig. 5.3).

\begin{tabular}{|cccc|cccc|}
\hline$X_{B J}$ & $g_{1}^{P}$ & $\sigma$ & sys & $X_{B J}$ & $g_{1}^{P}$ & $\sigma$ & sys \\
\hline 0.80551 & -0.00064 & 0.00060 & 0.00063 & 0.40475 & 0.14929 & 0.01635 & 0.01586 \\
0.76511 & -0.00042 & 0.00270 & 0.00099 & 0.38890 & 0.13387 & 0.01853 & 0.01195 \\
0.72732 & -0.00439 & 0.00532 & 0.00319 & 0.37392 & 0.17049 & 0.02429 & 0.01284 \\
0.69197 & -0.02842 & 0.00706 & 0.00620 & 0.35975 & 0.10450 & 0.02419 & 0.00969 \\
0.65888 & -0.00125 & 0.00639 & 0.00309 & 0.34633 & 0.11316 & 0.02495 & 0.01018 \\
0.62788 & 0.02138 & 0.00606 & 0.00684 & 0.33362 & 0.07554 & 0.02570 & 0.00956 \\
0.59883 & 0.03118 & 0.00621 & 0.00471 & 0.32156 & 0.13037 & 0.02694 & 0.01077 \\
0.57158 & 0.03079 & 0.00594 & 0.00501 & 0.31012 & 0.09208 & 0.02584 & 0.00980 \\
0.54601 & 0.04986 & 0.00835 & 0.01234 & 0.29926 & 0.17140 & 0.02863 & 0.00851 \\
0.52199 & 0.06490 & 0.00859 & 0.00721 & 0.28893 & 0.17636 & 0.02660 & 0.00907 \\
0.49940 & 0.11738 & 0.01084 & 0.01458 & 0.27911 & 0.15134 & 0.02719 & 0.00889 \\
0.47816 & 0.12169 & 0.01076 & 0.01972 & 0.26977 & 0.14428 & 0.02681 & 0.00832 \\
0.45816 & 0.12274 & 0.01218 & 0.01251 & 0.26087 & 0.14737 & 0.02650 & 0.00900 \\
0.43931 & 0.11274 & 0.01316 & 0.01968 & 0.25239 & 0.21534 & 0.04337 & 0.01308 \\
0.42153 & 0.12880 & 0.01646 & 0.02068 & 0.24431 & 0.26503 & 0.04664 & 0.01975 \\
\hline
\end{tabular}


Table C.10: $\Gamma_{1}^{P}$ vs. $\mathrm{Q}^{2}$ integrated up to $\mathrm{W}=2 \mathrm{GeV}$ (see Fig. 5.5).

\begin{tabular}{|cccc|}
\hline$Q^{2}\left(\mathrm{GeV}^{2}\right)$ & $\Gamma_{1}^{P}$ & $\sigma$ & sys \\
\hline 0.16612 & -0.00890 & 0.00473 & 0.00446 \\
0.20021 & -0.01206 & 0.00421 & 0.00522 \\
0.24530 & -0.00060 & 0.00382 & 0.00491 \\
0.29922 & -0.00456 & 0.00334 & 0.00523 \\
0.37026 & 0.00491 & 0.00246 & 0.00480 \\
0.47161 & 0.01401 & 0.00215 & 0.00543 \\
0.54927 & 0.01783 & 0.00201 & 0.00404 \\
0.66443 & 0.02234 & 0.00169 & 0.00373 \\
0.81183 & 0.02827 & 0.00161 & 0.00482 \\
0.98650 & 0.03102 & 0.00166 & 0.00368 \\
1.20262 & 0.03102 & 0.00169 & 0.00344 \\
1.46081 & 0.02557 & 0.00181 & 0.00279 \\
1.73878 & 0.02735 & 0.00185 & 0.00188 \\
2.04734 & 0.01961 & 0.00113 & 0.00146 \\
\hline
\end{tabular}


Table C.11: $\Gamma_{1}^{P}$ vs. $\mathrm{Q}^{2}$ integrated over full $\mathrm{W}$ phase space available (see Fig. 5.6).

\begin{tabular}{|cccc|}
\hline$Q^{2}\left(\mathrm{GeV}^{2}\right)$ & $\Gamma_{1}^{P}$ & $\sigma$ & sys \\
\hline 0.16612 & -0.00890 & 0.00473 & 0.00446 \\
0.20021 & -0.01206 & 0.00421 & 0.00522 \\
0.24530 & -0.00039 & 0.00384 & 0.00492 \\
0.29922 & -0.00419 & 0.00335 & 0.00524 \\
0.37026 & 0.01069 & 0.00261 & 0.00496 \\
0.47161 & 0.02069 & 0.00242 & 0.00562 \\
0.54927 & 0.02860 & 0.00235 & 0.00431 \\
0.66443 & 0.03430 & 0.00206 & 0.00402 \\
0.81183 & 0.03961 & 0.00196 & 0.00500 \\
0.98650 & 0.04504 & 0.00201 & 0.00400 \\
1.20262 & 0.04233 & 0.00196 & 0.00368 \\
1.46081 & 0.03391 & 0.00198 & 0.00294 \\
1.73878 & 0.03538 & 0.00200 & 0.00212 \\
2.04734 & 0.02592 & 0.00131 & 0.00177 \\
\hline
\end{tabular}


Table C.12: $\Gamma_{1}^{P}$ vs. $\mathrm{Q}^{2}$ integrated over full $\mathrm{W}$ phase space available with the deep inelastic contribution included (see Fig. 5.6).

\begin{tabular}{|cccc|}
\hline$Q^{2}\left(\mathrm{GeV}^{2}\right)$ & $\Gamma_{1}^{P}$ & $\sigma$ & sys \\
\hline 0.16612 & -0.00615 & 0.00473 & 0.00465 \\
0.20021 & -0.00801 & 0.00421 & 0.00510 \\
0.24530 & 0.00515 & 0.00384 & 0.00526 \\
0.29922 & 0.00407 & 0.00335 & 0.00557 \\
0.37026 & 0.01865 & 0.00261 & 0.00448 \\
0.47161 & 0.03274 & 0.00242 & 0.00449 \\
0.54927 & 0.04496 & 0.00235 & 0.00460 \\
0.66443 & 0.05572 & 0.00206 & 0.00440 \\
0.81183 & 0.06951 & 0.00196 & 0.00430 \\
0.98650 & 0.08272 & 0.00201 & 0.00401 \\
1.20262 & 0.09226 & 0.00196 & 0.00392 \\
1.46081 & 0.09790 & 0.00198 & 0.00392 \\
1.73878 & 0.10926 & 0.00200 & 0.00381 \\
2.04734 & 0.10929 & 0.00131 & 0.00379 \\
\hline
\end{tabular}


Table C.13: $I_{1}$ vs. $\mathrm{Q}^{2}$ integrated over full $\mathrm{W}$ phase space available (see Fig. 5.7).

\begin{tabular}{|cccc|}
\hline$Q^{2}\left(\mathrm{GeV}^{2}\right)$ & $I_{1}(\mathrm{mb})$ & $\sigma$ & sys \\
\hline 0.16612 & -74.22260 & 22.30880 & 0.08900 \\
0.20021 & -77.26306 & 15.97085 & 0.07967 \\
0.24530 & -35.70353 & 12.19060 & 0.06141 \\
0.29922 & -42.68106 & 8.99002 & 0.05348 \\
0.37026 & -19.18194 & 5.39955 & 0.03249 \\
0.47161 & 1.85860 & 3.51855 & 0.01807 \\
0.54927 & 8.02019 & 2.87244 & 0.01177 \\
0.66443 & 13.15724 & 1.97432 & 0.00619 \\
0.81183 & 16.63696 & 1.44566 & 0.00525 \\
0.98650 & 15.46194 & 1.12804 & 0.00293 \\
1.20262 & 13.85263 & 0.89601 & 0.00257 \\
1.46081 & 9.44530 & 0.78597 & 0.00174 \\
1.73878 & 9.04243 & 0.70219 & 0.00188 \\
2.04734 & 6.47302 & 0.43770 & 0.00130 \\
\hline
\end{tabular}


Table C.14: $I_{1}$ vs. $\mathrm{Q}^{2}$ integrated over full $\mathrm{W}$ phase space available with DIS contributed included (see Fig. 5.7).

\begin{tabular}{|cccc|}
\hline$Q^{2}\left(\mathrm{GeV}^{2}\right)$ & $I_{1}(\mathrm{mb})$ & $\sigma$ & sys \\
\hline 0.16612 & -67.93363 & 22.30880 & 0.02510 \\
0.20021 & -69.41034 & 15.97085 & 0.02270 \\
0.24530 & -25.69355 & 12.19060 & 0.02035 \\
0.29922 & -30.25225 & 8.99002 & 0.01804 \\
0.37026 & -4.02643 & 5.39955 & 0.01546 \\
0.47161 & 19.79865 & 3.51855 & 0.01238 \\
0.54927 & 27.34498 & 2.87244 & 0.01053 \\
0.66443 & 33.86251 & 1.97432 & 0.00847 \\
0.81183 & 38.35620 & 1.44566 & 0.00665 \\
0.98650 & 37.66812 & 1.12804 & 0.00526 \\
1.20262 & 36.04442 & 0.89601 & 0.00416 \\
1.46081 & 31.11952 & 0.78597 & 0.00333 \\
1.73878 & 29.87947 & 0.70219 & 0.00273 \\
2.04734 & 26.25605 & 0.43770 & 0.00227 \\
\hline
\end{tabular}




\section{References}

[1] M. Gell-Mann. Phys. Lett., 8, 214 (1964).

[2] CERN Reports No.8182/TH. 401 and No.8419/TH. 412 (1964), unpublished

[3] Y. Nambu and M.-Y. Han. Phys. Rev., D10, 674 (1974).

[4] O. W. Greenberg. Phys. Rev. Lett. 13, 598 (1964)

[5] H. Fritzsch, M. Gell-Mann, H. Leutwyler. Phys. Lett. B47, 365 (1973).

[6] J. D. Bjorken. Phys. Rev., 148, 1467 (1966); Phys. Rev., D1, 1376 (1970).

[7] D. E. Groom et al. The European Physical Journal, C15, 1 (2000).

[8] F. J. Gilman. Phys. Rev., 167, 1368 (1968).

[9] R. L. Jaffe and A. V. Manohar. Nucl.Phys., B337, 509 (1990).

[10] M. Beyer and S. K. Singh. Z. Phys. C, 31, 421 (1986).

[11] S. J. Brodsky and F. Schlumpf. Phys. Lett., textbfB32, 111 (1994).

[12] M. Anselmino, A. Efremov, and E. Leader. Phys.Rev. 261, 1 (1994).

[13] R. L. Jaffe and X. Ji. Phys. Rev., D54, 1 (1996). 
REFERENCES

[14] E. V. Shuryak and A. I. Vainshtein. Nucl. Phys. B199, 451 (1982); B201, 141 (1982).

[15] R. D. Erbacher, New F and D constants, E155, Technical Note 69,(1998), unpublished

[16] J. Ellis and R. Jaffe. Phys. Rev. D9, 1444 (1974); 10, 1669(E) (1974).

[17] EMC Collaboration, J. Ashman et al. Nucl. Phys., B328, 1 (1989).

[18] SMC Collaborations, D. Adams et al. Phys. Lett., B329, 399 (1994); Phys. Rev., D56, 5330 (1997).

[19] SMC Collaboration, B. Adeva et al. Phys. Lett., B302, 533 (1993); D. Adams et al., ibid. 357, 248 (1995); D. Adams et al., ibid. 396, 338 (1997).

[20] E142 Collaboration, P. L. Anthony et al. Phys. Rev. Lett. 71, 959 (1993); Phys. Rev., D54, 6620 (1990).

[21] E143 Collaboration, K. Abe et al. Phys. Rev., D58, 112003 (1998).

[22] R. D. Ball, S. Forte, G. Ridolfi. Nucl. Phys., B444, 287 (1995); B449, 680(E) (1995); Phys. Lett. B, 378, 255 (1996).

[23] S. D. Drell and A. C. Hearn. Phys. Rev. Lett., 16, 909 (1996).

[24] S. B. Gerasimov. Sov. J. Nucl. Phys., 2, 430 (1966).

[25] R. Van de Vyver for the GDH/A2 Collaboration. Phys. Rev. Lett., 87, 022003 (2001); J. Ahrens et al. Nucl. Phys., A689, 379 (2001). 
REFERENCES

[26] M. Gell-Mann and M. L. Goldberger. Phys. Rev., 96, 1433 (1954); F. E. Low. Phys. Rev., 96, 1428 (1954).

[27] M. Anselimo, B. L.Ioffe and E. Leader. Sov. J. Nucl. Phys., 49, 136 (1989).

[28] J. Ashman et al. Phys. Lett., 206B, 364 (1988).

[29] Hermes Collaboration, A. Airapetian et al. Phys.Lett., B494, 1 (2000).

[30] M. A. Ahmed and G. G. Ross. Nucl. Phys., B111, 441 (1976); K. Sasaki. Progr. Theor. Phys., 54, 1816 (1975); G. Altarelli and G. Parisi. Nucl. Phys., B126, 298 (1977).

[31] T. Gehrmann and W. J. Stirling. Phys. Rev., D53, 6100 (1996).

[32] J. Edelmann, G. Piller, N. Kaiser and W. Weise. Nucl. Phys., A665, 125 (2000).

[33] X. Ji. and P. Unrau. Phys. Rev., D52, 72 (1995).

[34] S. Simula, M. Osipendko, G. Ricco, M. Tauti. Leading and higher twists in the proton polarized structure function $g_{1}^{p}$ at large Bjorken $x$, preprint RM3TH/01-4 (2001).

[35] J. J. Sakurai. Currents and Mesons, University of Chicago Press, Chicago, 1969.

[36] V. Burkert and B. L. Ioffe. Phys.Lett., B296, 223 (1992); J.Exp.Theor.Phys., 78, 619 (1994).

[37] X. Ji and J. Osborne. J. Phys., G27, 127 (2001).

[38] S. A. Larin and J. A. M. Vermaseren. Phys. Lett., B259, 345 (1991), and references therein; S. A. Larin, ibid., 334, 192 (1994). 
[39] J. Soffer and O. Teryaev. Phys.Rev.Lett., 70, 3373 (1993); Phys. Rev., D51, 25 (1995).

[40] V. Burkert and Z. Li. Phys.Rev., D47, 46 (1993).

[41] D. Drechesel, O. Hanstein, S. S. Kamalov and L. Tiator. Nucl.Phys., A645, 145 (1999).

[42] R. L. Walker. Phys. Rev. 182, 1729 (1969).

[43] R. D. Moorehouse, in A. Donnachie and Shaw, Electromagnetic Interactions of Hadrons, N.Y. (1978).

[44] C. K. Sinclair. Report on Polarized Electron Source and Electron Polarimetery Workshop, 8th International Symposium on High Energy Spin Physics, AIP Conference Proceedings, 187(2) 1412 (1989).

[45] B. M. Dunham. Jefferson Lab Status Report JLAB-ACC-96-01 (1996).

[46] J. S. Price et al. Proceedings of 12th International Symposium on High-Energy Spin Physics, 10, (1996).

[47] J. Grames. Unique Electron Polarimeter Comparison and Spin-Based Energy Measurement, presented at PAC2001, Chicago, IL, 2001 (JLAB-ACC-01-12).

[48] B. Raue, L. Kramer, R. Nasseripour, F. Fortier. The Hall B Moller Polarimeter, CLAS technical note, unpublished.

[49] C. Cuevas, J. C. Denard, A. Freyberger, Y. Sharabian. Proceedings from talk presented at the 5th European Workship 
REFERENCES

on Diagnostics and Beam Instrumentation (2001) unpublished (www.esrf.fr/conferences/DIPAC/Proceedings/Proc_ContributedTalks.htm)

[50] Conceptual Design Report and Basic Experimental Equipment, (1990).

[51] M. D. Mestayer. et al., Nucl.Instr. and Meth. A449, 81 (2000).

[52] E.S. Smith. et al., Nucl.Instr. and Meth. A432, 265 (1999).

[53] G. Adams. et al., Submitted to Nucl. Instr. and Meth. (2000).

[54] M. Amarian. et al., Nucl.Instr. and Meth. A460, 239 (2000).

[55] M. Anghinolfi. et al., Nucl.Instr. and Meth. A447, 424 (1998).

[56] A. Abragam. eta al., Phys Lett., 2, 310 (1962).

[57] A. Abragam. Principles of Nuclear Magnetism (Oxford University Press, 1961).

[58] D. Crabb and W. Meyer. Ann.Rev.Nucl.Sci., 47, 67 (1997).

[59] M. Goldman. J. Magn. Res. 17, 393 (1975).

[60] D. Zimmermann. University of Virginia Polarized Taget Group Report (1993).

[61] G. Court. et al., Nucl. Instr. and Meth., A324, 433 (1993).

[62] B. Adeva. et al., Nucl. Instr. and Meth. A349, 334 (1994).

[63] F. Halzen and A. Martin. Quarks and Leptons: An Introductory Course to Modern Particle Physics (John Wiley \& Sons, 1984).

[64] S. Gasiorowiz. Quantum Physics (John Wiley \& Sons, 1974). 
[65] Raffaella Devita, Private Communication.

[66] Alex Skabelin, Private Communication.

[67] P. Bosted. Pair Symmetric Background Correction for EG1, unpublished.

[68] Volker Burkert, Private Communication.

[69] Linda Stuart, Radiative corrections program RCSLACPOL developed at SLAC and based on techniques by T.V. Kukhto and N. M. Shumeiko.

[70] S. Kuhn. Models for the Asymmetry A2, submitted to World Scientific on July 11,2001 .

[71] J. Soffer and O. V. Teryaev. hep-ph/9906455 Talk given at Workshop on Polarized Protons at High Energies - Accelerator Challenges and Physics Opportunities, Hamburg, Germany (1999).

[72] H. Burkhardt and W. N. Cottingham. Ann. Phys., 56, 453 (1970).

[73] T. V. Kukhto and N. M. Shumeiko. Nucl. Phys., B219, 412 (1983).

[74] Y. S. Tsai. Rev. Mod. Phys., 46, 815 (1974).

[75] A. Bodek et al., Phys. Lett. B282, 475 (1992).

[76] G. Ricco et al., Nucl. Phys. B555, 306 (1999).

[77] T. Averett. E155 Tech Note 27 (March 1997) Unpublished.

[78] The E142 Collaboration. Phys.Rev., D49, 4348 (1994).

[79] G.Gomes et al. Phys. Rev. D49, 4348 (1994) 
[80] P. L. Anthony et al., Phys. Lett. B493, 19 (2000).

[81] C.Y. Prescott. et al., Phys.Lett., B84, 524 (1979).

[82] P. Bosted and M. Peskin. Private Communication

[83] R. DeVita. Measurement of the Double Spin Asymmetry in $\pi^{+}$Electroproduction with CLAS. Ph.D Thesis, unpublished (2001). 